\title{
Diastereoselective Synthesis of Arabino- and Ribo-like Nucleoside Analogues bearing a Stereogenic C3'All-Carbon Quaternary Center
}

Tommy Lussier ${ }^{\dagger, \ddagger}$, Amarender Manchoju ${ }^{\dagger, \ddagger}$, Gang Wang ${ }^{\dagger, \ddagger}$, Starr Dostie ${ }^{\dagger}$, Scott Foster ${ }^{\dagger}$, Philippe Mochirian ${ }^{\dagger}$, Michel Prévost ${ }^{\dagger *}$, Yvan Guindon ${ }^{\dagger, \ddagger, \S^{*}}$

†Bio-organic Chemistry Laboratory, Institut de Recherches Cliniques de Montréal, Montréal, Québec H2W 1R7, Canada

‡Department of Chemistry, Université de Montréal, Montréal, Québec H3C 3J7, Canada

${ }^{\S}$ Department of Biochemistry, Microbiology and Immunology, University of Ottawa, Ottawa, Ontario K1N 6N5, Canada

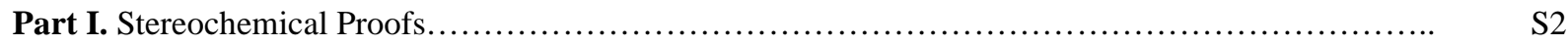

Part II. Computational Data........................................................ S3

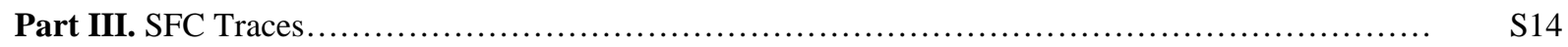

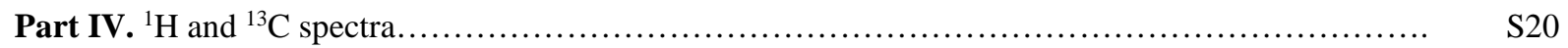

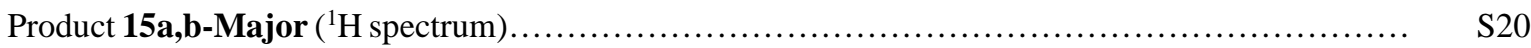

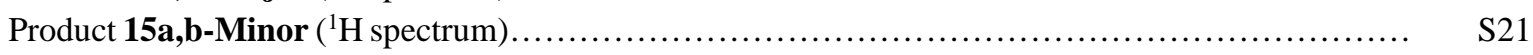

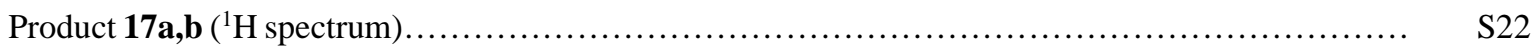

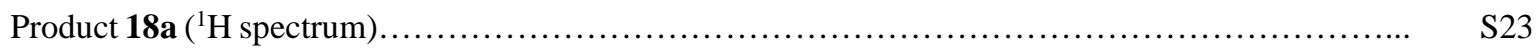

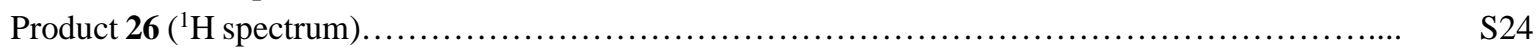

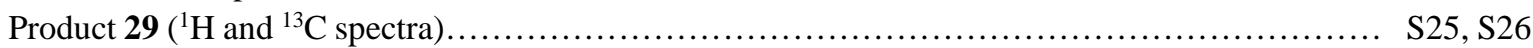

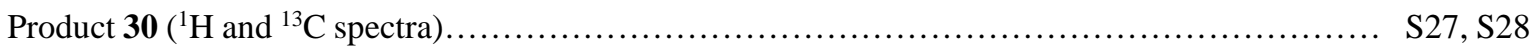

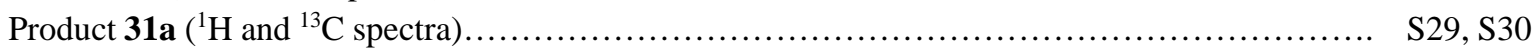

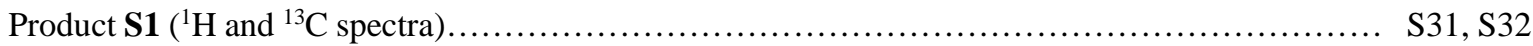

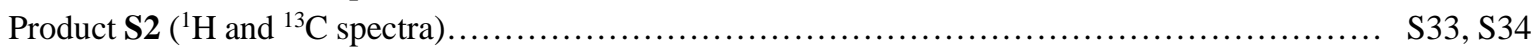

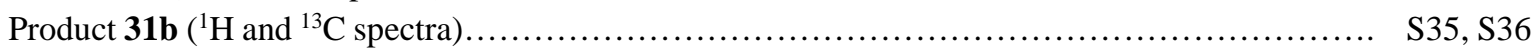

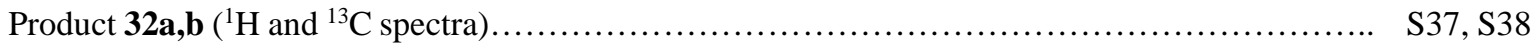

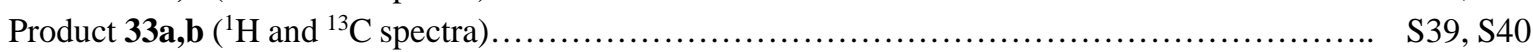

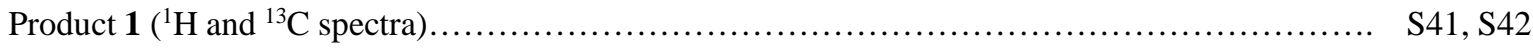

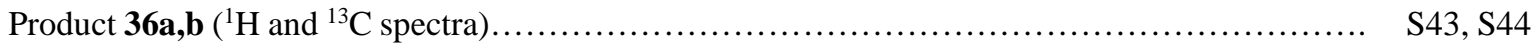

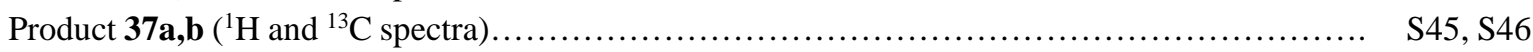

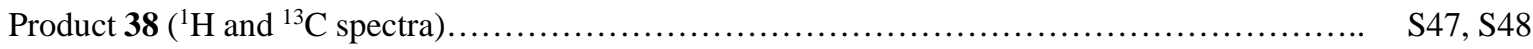

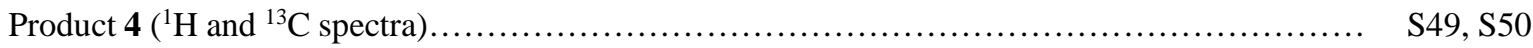

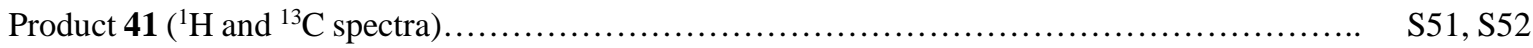

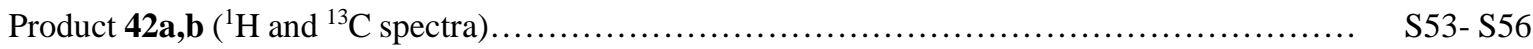

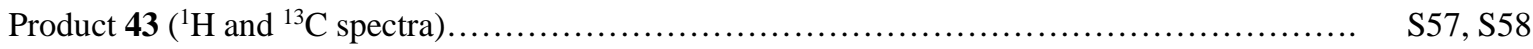

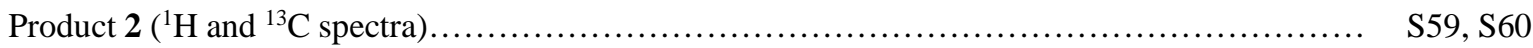

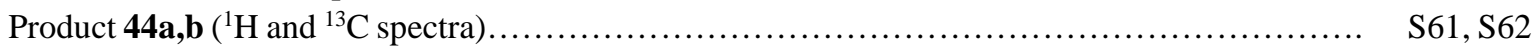

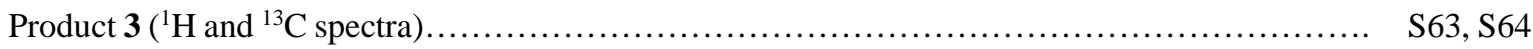




\section{$\underline{\text { Part I. Stereochemical Proofs }}$}

The relative configurations of protected and deprotected nucleoside analogues 41, 43, 3 and 4 were determined by relevant nuclear Overhauser effect (nOe) enhancements (2D NOESY). The peaks in the ${ }^{1} \mathrm{H}$ NMR spectra were assigned using ${ }^{1} \mathrm{H} /{ }^{1} \mathrm{H}$ 2D COSY experiments, chemical shifts, and coupling constants.
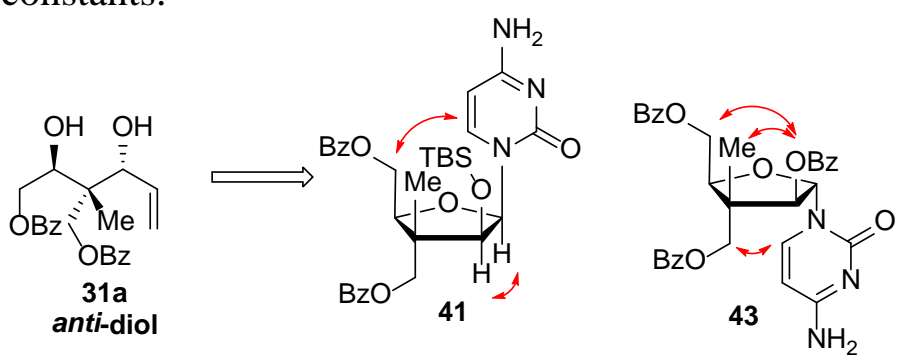

$=$ strong NOE correlation

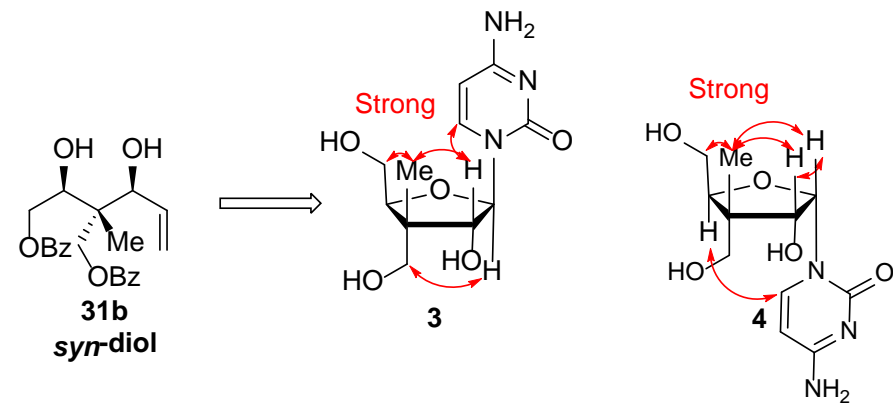

Acetonides of both the syn- and anti-diols (31a and 31b) were synthesized and separated by flash chromatography. Nuclear Overhauser effect (nOe) enhancements (2D NOESY) did not, however, allow to unambiguously assign their relative configurations. We assumed the relative configurations of 31a and 31b were not altered during the conversion to the nucleoside analogue scaffolds $\mathbf{4 1}, \mathbf{4 3}, \mathbf{3}$, and $\mathbf{4}$. It should be noted that formation of acetonide derivatives greatly facilitates the separation of 31a,b.
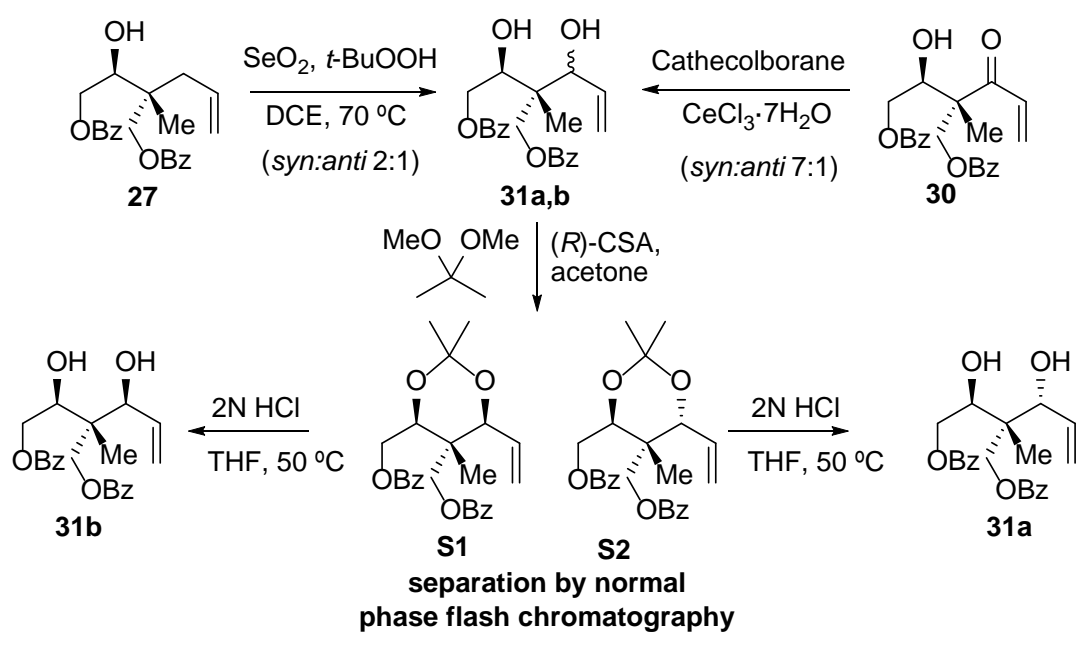


\section{Part II. Computational Data}

\section{DFT Method and Details}

Calculations were performed with Gaussian 09. ${ }^{1}$ The energies presented in the paper and the supporting information are from fully optimized structures at the BHandHLYP ${ }^{2,3}$ level of theory using the $\mathrm{TZVP}^{4}$ basis set for geometry optimizations. The different conformations of rotable bonds and enol radical geometries ( $\mathrm{E}$ or $\mathrm{Z}$ ) were examined and fully optimized for the different structures presented herein. The calculations in solvents (Toluene solvent employed for the radical initiation with $\mathrm{Et}_{3} \mathrm{~B}$ for the model compound in the calculations below) were achieved using the polarizable continuum model (PCM). ${ }^{5}$ Frequency calculations were performed on all optimized structures to characterize transient structures or intermediates, and to determine free energy values $\left(1\right.$ Hartree $\left.=627.5095 \mathrm{kcal} \mathrm{mol}^{-1}\right)$. Free energy corrections were applied using Truhlar's quasiharmonic approximation. ${ }^{6,7}$ Gibbs free energies of activation $\left(\Delta \Delta \mathrm{G}^{\ddagger}\right)$ correspond to the energy difference between the lowest energy predictive TS (273.15K, $1 \mathrm{~atm})$.

\section{Energies (Hartree) in Toluene (PCM) at the BHandHLYP/TZVP level of theory, imaginary vibrational frequencies, and Cartesian coordinates $(\AA \AA)$, of the optimized structures are presented below:}

Data for Scheme 5.

\begin{tabular}{|c|c|}
\hline $\begin{array}{r}\text { Structure } \\
\end{array}$ & $\mathrm{G}_{\text {rel }}\left(\mathrm{kcal} \cdot \mathrm{mol}^{-1}\right)$ \\
\hline 20c & 0 \\
\hline TS B & $(\mathrm{qG}$ TS B $-\mathrm{qG}$ 20c) $* 627.5095=16.9$ \\
\hline TS C & $(\mathrm{qG}$ TS C $-\mathrm{qG} 20 \mathrm{c}) * 627.5095=21.6$ \\
\hline
\end{tabular}

Data for Scheme 6.

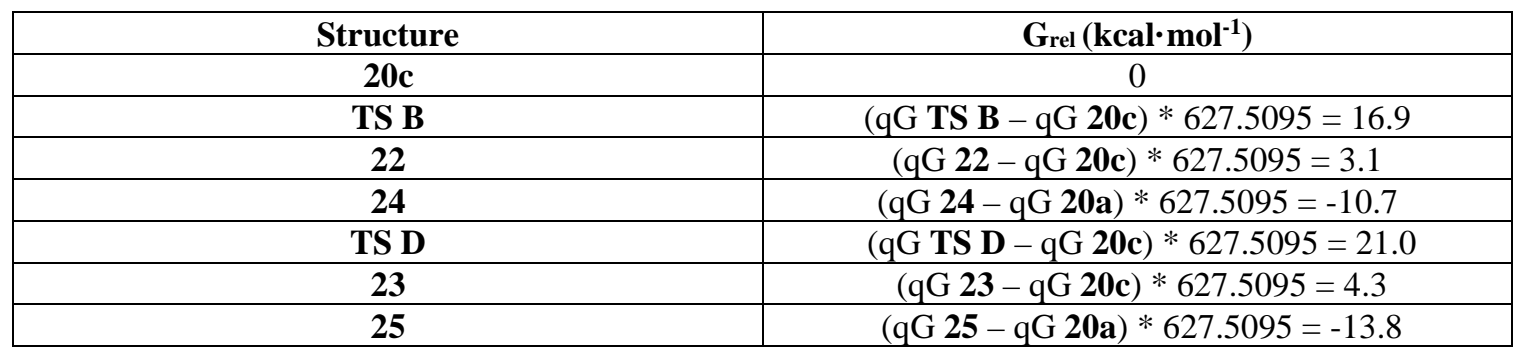

\section{Allylsilane tether}

20a

$\mathrm{E}=-3599.619555$ Hartree

$\mathrm{G}=-3599.294870$ Hartree

qG (G with quasiharmonic correction $)=-3599.290334$ Hartree

$\mathrm{N}_{\text {(imaginary) }}=0$

$\begin{array}{lrrr}\text { O } & -0.97386800 & 0.15385500 & -0.21718100 \\ \mathrm{C} & 0.23089600 & 0.67499000 & 0.25692600 \\ \mathrm{C} & 1.41102200 & 0.02838600 & -0.48812800 \\ \mathrm{Si} & -2.21532000 & -0.63177300 & 0.58220900\end{array}$




\begin{tabular}{lrrr} 
& & & \\
$\mathrm{C}$ & 0.25869100 & 2.22175300 & 0.13888200 \\
$\mathrm{C}$ & -2.55361800 & -2.23717500 & -0.31266600 \\
$\mathrm{C}$ & -1.76517000 & -0.92337200 & 2.37938100 \\
$\mathrm{C}$ & 1.37640500 & 0.19701100 & -1.98805700 \\
$\mathrm{O}$ & 2.72205700 & 0.44936400 & 0.16104100 \\
$\mathrm{O}$ & 2.81984000 & 0.78643900 & 1.30294100 \\
$\mathrm{H}$ & 3.74034300 & 0.39403400 & -0.67588000 \\
$\mathrm{H}$ & 0.36597200 & 0.42642100 & 1.30182300 \\
$\mathrm{H}$ & -1.70708700 & -2.90901300 & -0.23093700 \\
$\mathrm{H}$ & -3.42592200 & -2.73135000 & 0.10472900 \\
$\mathrm{H}$ & -2.74476300 & -2.05796000 & -1.36509700 \\
$\mathrm{H}$ & -0.86641100 & -1.52321900 & 2.47291200 \\
$\mathrm{H}$ & -2.57288600 & -1.45843900 & 2.86996900 \\
$\mathrm{H}$ & -1.61110100 & 0.00475800 & 2.91990900 \\
$\mathrm{H}$ & 0.40976500 & -0.10899500 & -2.36189300 \\
$\mathrm{H}$ & 1.53822300 & 1.23849000 & -2.24206300 \\
$\mathrm{Br}$ & 2.14901900 & -0.38648600 & -2.46437400 \\
$\mathrm{C}$ & 1.38437400 & -1.94002400 & -0.10644700 \\
$\mathrm{H}$ & -0.61311100 & 2.76710400 & -0.98393100 \\
$\mathrm{H}$ & -0.44997300 & 3.83543200 & -1.08088700 \\
$\mathrm{H}$ & -1.66204700 & 2.60924900 & -0.76861800 \\
$\mathrm{H}$ & -0.40409200 & 2.30851400 & -1.94102700 \\
$\mathrm{C}$ & 1.28263700 & 2.52465000 & -0.05968400 \\
$\mathrm{H}$ & -0.13817000 & 2.85104300 & 1.46824100 \\
$\mathrm{H}$ & 0.53791900 & 2.55135300 & 2.26063500 \\
$\mathrm{H}$ & -1.14533200 & 2.55801300 & 1.75122100 \\
$\mathrm{C}$ & -0.12095700 & 3.93361400 & 1.40067400 \\
$\mathrm{H}$ & 5.02662200 & 0.69851600 & -0.14124200 \\
$\mathrm{H}$ & 5.04656100 & 1.71032200 & 0.23961800 \\
$\mathrm{H}$ & 5.71614900 & 0.58962600 & -0.96298500 \\
$\mathrm{C}$ & 5.27297600 & 0.00894600 & 0.65451000 \\
$\mathrm{H}$ & -3.74455300 & 0.48506700 & 0.50398200 \\
$\mathrm{H}$ & -4.52741600 & 0.01846300 & 1.09744600 \\
$\mathrm{C}$ & -3.48549600 & 1.41835500 & 1.00137200 \\
$\mathrm{H}$ & -4.23555800 & 0.75923700 & -0.87493600 \\
$\mathrm{C}$ & -3.57651600 & 1.33830000 & -1.50414900 \\
$\mathrm{H}$ & -5.37823200 & 0.33744700 & -1.38474600 \\
$\mathrm{H}$ & -5.65470900 & 0.55883600 & -2.40040800 \\
& -6.07734000 & -0.24305000 & -0.80550400 \\
& & & \\
\hline
\end{tabular}

\section{0c}

$\mathrm{E}=-1025.441255$ Hartree

$G=-1025.121716$ Hartree

qG $(\mathrm{G}$ with quasiharmonic correction $)=-1025.115701$ Hartree

$\mathrm{N}_{\text {(imaginary) }}=0$

$\begin{array}{lrrr}\mathrm{O} & -0.82857000 & 0.69869200 & -0.25686200 \\ \mathrm{C} & 0.55188800 & 0.95476700 & -0.08084100 \\ \mathrm{C} & 1.34182000 & -0.20969700 & -0.59443100 \\ \mathrm{Si} & -1.84985000 & -0.02198800 & 0.85194800 \\ \mathrm{C} & 0.90334300 & 2.26153700 & -0.80251100 \\ \mathrm{C} & -1.22622600 & -1.72501300 & 1.32491500 \\ \mathrm{C} & -1.99957300 & 1.05568400 & 2.38024300 \\ \mathrm{C} & 1.11575200 & -0.71639100 & -1.96930000 \\ \mathrm{C} & 2.34304100 & -0.80017700 & 0.26462000 \\ \mathrm{O} & 2.55395100 & -0.46621500 & 1.40714600 \\ \mathrm{O} & 3.03344300 & -1.77648500 & -0.33274100 \\ \mathrm{H} & 0.78516000 & 1.08200600 & 0.97247200 \\ \mathrm{H} & -0.26637800 & -1.66421400 & 1.82806300 \\ \mathrm{H} & -1.92367600 & -2.20624700 & 2.00427600\end{array}$




$\begin{array}{lrrr}\mathrm{H} & -1.11688200 & -2.36107900 & 0.45317200 \\ \mathrm{H} & -1.04621000 & 1.15458500 & 2.88949200 \\ \mathrm{H} & -2.70288200 & 0.62180800 & 3.08474800 \\ \mathrm{H} & -2.34963900 & 2.05037400 & 2.12460200 \\ \mathrm{H} & 0.21373800 & -0.29204600 & -2.38732800 \\ \mathrm{H} & 1.95242800 & -0.46400500 & -2.61909600 \\ \mathrm{H} & 1.03438300 & -1.79763100 & -1.98312500 \\ \mathrm{C} & 0.09646200 & 3.41953100 & -0.23607500 \\ \mathrm{H} & 0.31568300 & 4.33416500 & -0.77696900 \\ \mathrm{H} & 0.34412300 & 3.58619400 & 0.80910200 \\ \mathrm{H} & -0.96661000 & 3.23129300 & -0.30693600 \\ \mathrm{H} & 0.62673000 & 2.13075600 & -1.84633900 \\ \mathrm{C} & 2.39519500 & 2.55575900 & -0.72439800 \\ \mathrm{H} & 2.99057000 & 1.79124400 & -1.21159700 \\ \mathrm{H} & 2.72528000 & 2.62572700 & 0.30772800 \\ \mathrm{H} & 2.61736800 & 3.50053200 & -1.20813000 \\ \mathrm{C} & 4.03349100 & -2.41643600 & 0.44665600 \\ \mathrm{H} & 4.78017300 & -1.70339500 & 0.77052800 \\ \mathrm{H} & 4.47886300 & -3.15809200 & -0.19820700 \\ \mathrm{H} & 3.59546400 & -2.88974100 & 1.31565100 \\ \mathrm{C} & -3.51180300 & -0.11461200 & -0.03952600 \\ \mathrm{H} & -4.23459500 & -0.56152400 & 0.63870200 \\ \mathrm{H} & -3.82996400 & 0.91130700 & -0.21493400 \\ \mathrm{C} & -3.46892400 & -0.86280300 & -1.32642500 \\ \mathrm{H} & -2.90706200 & -0.39489000 & -2.12031600 \\ \mathrm{C} & -4.03172900 & -2.03501300 & -1.55652900 \\ \mathrm{H} & -3.93956800 & -2.52370300 & -2.51033900 \\ \mathrm{H} & -4.60561400 & -2.54634800 & -0.80122600\end{array}$

The fully minimized structure adopts a planar conformation with most NBO spin density on the alpha carbon (0.80) and on the enol-oxygen (0.18).

\section{TS B}

$E=-1025.416808$ Hartree

$G=-1025.089624$ Hartree

qG $(G$ with quasiharmonic correction $)=-1025.087636$ Hartree

$\mathrm{N}_{\text {(imaginary) }}=1, v=-538.6 \mathrm{i} \mathrm{cm}{ }^{-1}$

$\begin{array}{lrrr}\mathrm{O} & 1.27678600 & 0.41607500 & 0.64886500 \\ \mathrm{C} & 0.03180900 & 0.76539000 & 0.07173600 \\ \mathrm{C} & -1.07396500 & -0.11966500 & 0.60631200 \\ \mathrm{C} & -0.42464000 & -2.18589900 & 0.52099600 \\ \mathrm{C} & 0.55513300 & -2.41467200 & -0.40953000 \\ \mathrm{C} & 1.99893400 & -2.26001000 & -0.10802000 \\ \mathrm{Si} & 2.49711400 & -0.42684900 & -0.10714200 \\ \mathrm{C} & 4.02880700 & -0.15770300 & 0.93086400 \\ \mathrm{C} & 2.74844300 & 0.16525100 & -1.86937200 \\ \mathrm{C} & -1.37407000 & -0.01742900 & 2.06992300 \\ \mathrm{C} & -0.20882700 & 2.25848900 & 0.35749200 \\ \mathrm{C} & -2.18519000 & -0.32084500 & -0.32524900 \\ \mathrm{O} & -2.11356000 & -0.22338200 & -1.52421300 \\ \mathrm{O} & -3.31786100 & -0.68840100 & 0.28525800 \\ \mathrm{C} & -4.42910800 & -0.97084000 & -0.55262600 \\ \mathrm{H} & 0.06444800 & 0.63218200 & -1.00483600 \\ \mathrm{H} & -1.42001900 & -2.54120300 & 0.32529700 \\ \mathrm{H} & -0.14475400 & -2.16773700 & 1.55967400 \\ \mathrm{H} & 0.26800600 & -2.60602700 & -1.43028100 \\ \mathrm{H} & 2.22261800 & -2.64706100 & 0.88344300 \\ \mathrm{H} & 2.61402600 & -2.80280900 & -0.81875300\end{array}$




$\begin{array}{lrrr}\mathrm{H} & 3.84738100 & -0.43969600 & 1.96261700 \\ \mathrm{H} & 4.32889800 & 0.88488200 & 0.91501100 \\ \mathrm{H} & 4.85772600 & -0.75224100 & 0.55916900 \\ \mathrm{H} & 3.59500000 & -0.35306000 & -2.31024400 \\ \mathrm{H} & 2.95562700 & 1.22954300 & -1.90143600 \\ \mathrm{H} & 1.88332100 & -0.02862100 & -2.49441300 \\ \mathrm{H} & -0.44891600 & 0.01269900 & 2.63242600 \\ \mathrm{H} & -1.96819400 & -0.85224400 & 2.41626600 \\ \mathrm{H} & -1.93165600 & 0.88833100 & 2.29715600 \\ \mathrm{H} & -0.17199400 & 2.37763700 & 1.43744600 \\ \mathrm{H} & -5.23378600 & -1.24962500 & 0.11036400 \\ \mathrm{H} & -4.20197500 & -1.78423000 & -1.22931000 \\ \mathrm{H} & -4.70463400 & -0.09837100 & -1.13000800 \\ \mathrm{C} & -1.56191100 & 2.74984400 & -0.14027600 \\ \mathrm{H} & -1.66154100 & 3.81258000 & 0.05276600 \\ \mathrm{H} & -2.39242700 & 2.25505400 & 0.35017400 \\ \mathrm{H} & -1.66700000 & 2.59356100 & -1.20870900 \\ \mathrm{C} & 0.90275500 & 3.10679000 & -0.24461800 \\ \mathrm{H} & 0.91434800 & 3.01618900 & -1.32764700 \\ \mathrm{H} & 1.87391500 & 2.81580300 & 0.13309100 \\ \mathrm{H} & 0.75004500 & 4.15382100 & -0.00434400\end{array}$

TS C

$\mathrm{E}=-1025.409399$ Hartree

$\mathrm{G}=-1025.082099$ Hartree

qG (G with quasiharmonic correction $)=-1025.0801$ Hartree $\mathrm{N}_{\text {(imaginary) }}=1, v=-543.5 \mathrm{i} \mathrm{cm}^{-1}$

$\begin{array}{lrrr}\mathrm{O} & -0.99168800 & 0.31789400 & -0.59541700 \\ \mathrm{C} & -0.04285500 & 0.82106600 & 0.31899400 \\ \mathrm{C} & 1.10136600 & -0.14885000 & 0.59317700 \\ \mathrm{C} & 0.41706600 & -2.19248200 & 0.37748100 \\ \mathrm{C} & -0.83767600 & -2.37809600 & 0.89734600 \\ \mathrm{C} & -2.07132200 & -2.24470800 & 0.08610500 \\ \mathrm{Si} & -2.44185200 & -0.41236900 & -0.24957300 \\ \mathrm{C} & -3.51011100 & -0.21947900 & -1.77113300 \\ \mathrm{C} & -3.25043400 & 0.36184900 & 1.25832000 \\ \mathrm{C} & 1.54997700 & -0.18830600 & 2.02700700 \\ \mathrm{C} & 0.39398600 & 2.20587600 & -0.19272800 \\ \mathrm{C} & 2.11662500 & -0.29614400 & -0.45336600 \\ \mathrm{O} & 1.97574700 & -0.01208200 & -1.61384500 \\ \mathrm{O} & 3.25009700 & -0.85246200 & -0.00028600 \\ \mathrm{C} & 4.26365000 & -1.09740100 & -0.96337500 \\ \mathrm{H} & -0.52549600 & 0.97870200 & 1.28352300 \\ \mathrm{H} & 1.26819700 & -2.52471700 & 0.94199800 \\ \mathrm{H} & 0.52590200 & -2.22120700 & -0.69319900 \\ \mathrm{H} & -0.94456800 & -2.52177700 & 1.96038500 \\ \mathrm{H} & -1.94114200 & -2.71819900 & -0.88424400 \\ \mathrm{H} & -2.92191500 & -2.71346500 & 0.57009900 \\ \mathrm{H} & -2.98882300 & -0.58437700 & -2.64972900 \\ \mathrm{H} & -3.76219200 & 0.82244400 & -1.93890900 \\ \mathrm{H} & -4.43534800 & -0.77841300 & -1.66921300 \\ \mathrm{H} & -4.23962800 & -0.06347400 & 1.40001800 \\ \mathrm{H} & -3.36452300 & 1.43407600 & 1.13655800 \\ \mathrm{H} & -2.68485800 & 0.18375600 & 2.16688300 \\ \mathrm{H} & 0.70367900 & -0.39288700 & 2.67389000 \\ \mathrm{H} & 1.97146500 & 0.76283300 & 2.33962400 \\ \mathrm{H} & 2.30190400 & -0.94534500 & 2.19417700 \\ \mathrm{H} & 0.76091900 & 2.05254300 & -1.20037200 \\ \mathrm{H} & 5.08074300 & -1.54986100 & -0.42241400\end{array}$




$\begin{array}{lrrr}\mathrm{H} & 4.58624400 & -0.17287600 & -1.42384100 \\ \mathrm{H} & 3.90692900 & -1.76827600 & -1.73380200 \\ \mathrm{C} & 1.50209300 & 2.83982900 & 0.63694200 \\ \mathrm{H} & 1.73940000 & 3.82136000 & 0.24128100 \\ \mathrm{H} & 2.41710400 & 2.25944400 & 0.62617500 \\ \mathrm{H} & 1.19858000 & 2.97175100 & 1.67222300 \\ \mathrm{C} & -0.80382200 & 3.14428300 & -0.25212400 \\ \mathrm{H} & -1.21210400 & 3.31916600 & 0.74090000 \\ \mathrm{H} & -1.58991400 & 2.74441600 & -0.87865800 \\ \mathrm{H} & -0.50914600 & 4.10611400 & -0.65883100\end{array}$

\section{TS D}

$\mathrm{E}=-1025.408979$ Hartree

$\mathrm{G}=-1025.082819$ Hartree

qG (G with quasiharmonic correction $)=-1025.081021$ Hartree

$\mathrm{N}_{\text {(imaginary) }}=1, v=-588.9 \mathrm{i} \mathrm{cm}^{-1}$

$\begin{array}{lrrr}\mathrm{O} & -1.47954700 & 0.80993100 & -0.35254300 \\ \mathrm{C} & -0.14987900 & 0.97915600 & 0.09394000 \\ \mathrm{C} & 0.74918700 & -0.13125800 & -0.42526300 \\ \mathrm{C} & -0.08378200 & -1.96457000 & 0.35870700 \\ \mathrm{C} & -1.47509100 & -2.01716900 & -0.22649100 \\ \mathrm{Si} & -2.47258700 & -0.45252600 & 0.07712600 \\ \mathrm{C} & -3.97262100 & -0.34290400 & -1.03045900 \\ \mathrm{C} & -2.95237600 & -0.32033600 & 1.88645400 \\ \mathrm{C} & 0.82389900 & -2.95331100 & 0.04332500 \\ \mathrm{C} & 0.79083300 & -0.30924400 & -1.91078300 \\ \mathrm{C} & 2.00954000 & -0.26649000 & 0.32202700 \\ \mathrm{O} & 2.12916900 & -0.07597200 & 1.50467500 \\ \mathrm{O} & 3.02818600 & -0.67351300 & -0.43537600 \\ \mathrm{C} & 4.26534000 & -0.89363900 & 0.22731000 \\ \mathrm{H} & -0.11374600 & 0.95732900 & 1.18080900 \\ \mathrm{H} & -0.00149100 & -1.55493500 & 1.35295800 \\ \mathrm{H} & -1.99941700 & -2.86449800 & 0.21289100 \\ \mathrm{H} & -1.42096100 & -2.21867300 & -1.29295100 \\ \mathrm{H} & -4.50614500 & 0.58723800 & -0.86450200 \\ \mathrm{H} & -4.65890100 & -1.16130000 & -0.83555300 \\ \mathrm{H} & -3.68551100 & -0.38378600 & -2.07576300 \\ \mathrm{H} & -3.61911900 & -1.13064700 & 2.16605300 \\ \mathrm{H} & -3.46676700 & 0.61623800 & 2.07695800 \\ \mathrm{H} & -2.08812100 & -0.36384600 & 2.54130200 \\ \mathrm{H} & 0.69105200 & -3.57153100 & -0.82723700 \\ \mathrm{H} & 1.73563100 & -3.07555100 & 0.59944700 \\ \mathrm{H} & 1.37675500 & 0.47780600 & -2.37993300 \\ \mathrm{H} & -0.20873900 & -0.26070100 & -2.32265600 \\ \mathrm{H} & 1.24141100 & -1.25268400 & -2.18578300 \\ \mathrm{H} & 4.95489900 & -1.21734400 & -0.53693600 \\ \mathrm{H} & 4.16143900 & -1.65727500 & 0.98705400 \\ \mathrm{H} & 4.61787300 & 0.01834500 & 0.69025700 \\ \mathrm{C} & 0.26948600 & 2.38811900 & -0.36680100 \\ \mathrm{H} & 0.08753100 & 2.42040600 & -1.43811900 \\ \mathrm{C} & 1.73269000 & 2.72245800 & -0.11478700 \\ \mathrm{H} & 1.93044700 & 3.74236200 & -0.42666600 \\ \mathrm{H} & 2.40929300 & 2.08256500 & -0.66995600 \\ \mathrm{H} & 1.98213800 & 2.64184800 & 0.93767100 \\ & -0.62348700 & 3.43037000 & 0.29386800 \\ \mathrm{H} & -1.66934000 & 3.22836100 & 0.10697900 \\ \mathrm{H} & -0.46456900 & 3.44415100 & 1.36918600\end{array}$


23

$E=-1025.437682$ Hartree

$G=-1025.109295$ Hartree

qG $(\mathrm{G}$ with quasiharmonic correction $)=-1025.1076$ Hartree

$\mathrm{N}_{\text {(imaginary) }}=0$

$\begin{array}{llll}\text { O } & 1.38914800 & 0.93691200 & 0.18292200\end{array}$

$\begin{array}{llll}\text { C } & -0.00138800 & 0.88037200 & -0.06557600\end{array}$

$\begin{array}{llll}\text { C } & -0.60074800 & -0.42075300 & 0.55526800\end{array}$

$\begin{array}{llll}\text { C } & 0.08172600 & -1.67965100 & -0.08761800\end{array}$

C $\quad \begin{array}{llll}\text { C } & 1.58266300 & -1.82105700 & 0.25711800\end{array}$

Si $\quad 2.48624200 \quad-0.24750000 \quad-0.22274100$

$\begin{array}{llll}\text { C } & 4.03084500 & 0.08816000 & 0.77182200\end{array}$

$\begin{array}{llll}\text { C } & 2.84988500 & -0.20879300 & -2.06335900\end{array}$

$\begin{array}{llll}\text { C } & -0.63149100 & -2.94586900 & 0.23450400\end{array}$

$\begin{array}{llll}\text { C } & -0.46192800 & -0.41256500 & 2.07188100\end{array}$

$\begin{array}{llll}\text { C } & -2.06920800 & -0.57412600 & 0.18951500\end{array}$

$\begin{array}{llll}\mathrm{O} & -2.94835400 & -0.81907000 & 0.96220000\end{array}$

$\begin{array}{llll}\text { O } & -2.27924800 & -0.45821800 & -1.11807400\end{array}$

C $\quad-3.61272300 \quad-0.65571200-1.57181900$

$\mathrm{H} \quad-0.17365800 \quad 0.82998100 \quad-1.13940800$

$\mathrm{H} \quad 0.01385100 \quad-1.53657200 \quad-1.16466300$

$\mathrm{H} \quad 1.97630100 \quad-2.68659900 \quad-0.26734200$

$\mathrm{H} \quad \begin{array}{llll}\mathrm{H} & 1.70331700 & -2.02095800 & 1.31728000\end{array}$

$\mathrm{H} \quad 4.44673400 \quad 1.05781000 \quad 0.51757500$

$\begin{array}{llll}\mathrm{H} & 4.78966900 & -0.66279300 & 0.57451300\end{array}$

$\mathrm{H} \quad 3.81605500 \quad 0.08189900 \quad 1.83493600$

$\mathrm{H} \quad 3.57532000 \quad-0.97250900 \quad-2.32770500$

$\mathrm{H} \quad 3.25851400 \quad 0.75423900 \quad-2.35269900$

$\mathrm{H} \quad 1.95614800 \quad-0.38267600 \quad-2.65396200$

$\mathrm{H} \quad-0.39091900-3.83221500-0.32410600$

$\mathrm{H} \quad \begin{array}{llll}-1.24942300 & -3.05291400 & 1.10608800\end{array}$

$\mathrm{H} \quad-1.11325400 \quad 0.32753100 \quad 2.51709200$

$\begin{array}{llll}\mathrm{H} & 0.55585000 & -0.17967600 & 2.35332500\end{array}$

$\mathrm{H} \quad-0.72765500 \quad-1.37023500 \quad 2.49907400$

$\mathrm{H} \quad-3.58169100 \quad-0.52383400 \quad-2.64218900$

$\mathrm{H} \quad-4.27775100 \quad 0.06914900 \quad-1.12175700$

$\mathrm{H} \quad \begin{array}{llll}-3.95187700 & -1.65258500 & -1.32439400\end{array}$

$\begin{array}{llll}\text { C } & -0.55784700 & 2.21898100 & 0.44235000\end{array}$

$\begin{array}{llll}\mathrm{H} & -0.28491100 & 2.28926200 & 1.49169900\end{array}$

$\begin{array}{llll}\text { C } & -2.06713100 & 2.38813000 & 0.32408800\end{array}$

$\mathrm{H} \quad-2.33840400 \quad 3.39345100 \quad 0.62763100$

$\mathrm{H} \quad-2.62504600 \quad 1.70756800 \quad 0.95323500$

$\mathrm{H} \quad-2.39938100 \quad 2.25855400 \quad-0.70122000$

$\begin{array}{llll}\text { C } & 0.13029500 & 3.36356000 & -0.29478800\end{array}$

$\begin{array}{llll}\mathrm{H} & 1.20522400 & 3.30850100 & -0.19920100\end{array}$

$\mathrm{H} \quad \begin{array}{llll}\mathrm{H} & -0.20108000 & 4.31785200 & 0.10085700\end{array}$

$\begin{array}{llll}\mathrm{H} & -0.11940100 & 3.34169900 & -1.35298600\end{array}$

22

$E=-1025.441328$ Hartree

$G=-1025.111332$ Hartree

qG $(\mathrm{G}$ with quasiharmonic correction $)=-1025.1095$ Hartree

$\mathrm{N}_{(\text {imaginary })}=0$

\begin{tabular}{|c|c|c|c|}
\hline D & 1.09132800 & 0.66601400 & 0.36398000 \\
\hline C & -0.09214200 & 0.48135300 & -0.37386600 \\
\hline C & -0.85767800 & -0.78486300 & \\
\hline C & -0.31281500 & -2.07442600 & -0.556 \\
\hline C & 1.13985500 & -2.16000100 & -0.87546 \\
\hline
\end{tabular}




$\begin{array}{lrrr}\mathrm{C} & 2.23422400 & -1.93963600 & 0.09436300 \\ \mathrm{Si} & 2.57019900 & -0.07316500 & 0.16610200 \\ \mathrm{C} & 3.58623700 & 0.43487400 & 1.65145900 \\ \mathrm{C} & 3.37646400 & 0.48718500 & -1.43165400 \\ \mathrm{C} & -0.75853000 & -0.94144300 & 1.66195100 \\ \mathrm{C} & -0.85007300 & 1.81789800 & -0.39199400 \\ \mathrm{C} & -2.30757100 & -0.65361400 & -0.30511600 \\ \mathrm{O} & -2.67023700 & -0.77599000 & -1.44057200 \\ \mathrm{O} & -3.15082400 & -0.36730300 & 0.67741700 \\ \mathrm{C} & -4.51688200 & -0.19148300 & 0.31896900 \\ \mathrm{H} & 0.14914700 & 0.26724700 & -1.41330200 \\ \mathrm{H} & -0.85487300 & -2.19344600 & -1.48448800 \\ \mathrm{H} & -0.59877300 & -2.91426900 & 0.07963900 \\ \mathrm{H} & 1.40852900 & -2.31259500 & -1.90593700 \\ \mathrm{H} & 1.97662600 & -2.28985900 & 1.09089100 \\ \mathrm{H} & 3.14089700 & -2.45517700 & -0.21091900 \\ \mathrm{H} & 3.10232500 & 0.13185500 & 2.57381400 \\ \mathrm{H} & 3.71519000 & 1.51210400 & 1.67761600 \\ \mathrm{H} & 4.57124900 & -0.02050000 & 1.62041000 \\ \mathrm{H} & 4.35191300 & 0.02455400 & -1.54979900 \\ \mathrm{H} & 3.51472300 & 1.56357400 & -1.43493000 \\ \mathrm{H} & 2.77714000 & 0.22306800 & -2.29647000 \\ \mathrm{H} & 0.26372200 & -1.13253600 & 1.95142400 \\ \mathrm{H} & -1.36122900 & -1.78129100 & 1.98684100 \\ \mathrm{H} & -1.09282800 & -0.06442600 & 2.19385100 \\ \mathrm{H} & -1.77592300 & 1.65181200 & -0.93529400 \\ \mathrm{H} & -5.03704600 & 0.01611000 & 1.24096000 \\ \mathrm{H} & -4.90668200 & -1.08917500 & -0.14116500 \\ \mathrm{H} & -4.62443900 & 0.63625300 & -0.36924100 \\ \mathrm{C} & -0.04420300 & 2.83697500 & -1.19038200 \\ \mathrm{H} & -0.60056200 & 3.76225800 & -1.29508600 \\ \mathrm{H} & 0.18313800 & 2.47105500 & -2.18670700 \\ \mathrm{H} & 0.89144700 & 3.06325000 & -0.69172700 \\ \mathrm{C} & -1.18518600 & 2.38086200 & 0.98320800 \\ \mathrm{H} & -0.29139400 & 2.46492900 & 1.59032000 \\ \mathrm{H} & -1.90573800 & 1.77239700 & 1.51317600 \\ \mathrm{H} & -1.61167900 & 3.37327500 & 0.87788900\end{array}$

\section{5}

$\mathrm{E}=-3599.647378$ Hartree

$G=-3599.315252$ Hartree

qG (G with quasiharmonic correction $)=-3599.312311$ Hartree

$\mathrm{N}_{\text {(imaginary) }}=0$

$\begin{array}{lrrr}\text { O } & 1.93840600 & 1.30293900 & -0.13534500 \\ \mathrm{C} & 1.75631000 & -0.07297800 & 0.11807200 \\ \mathrm{C} & 0.50884800 & -0.60671100 & -0.66501100 \\ \mathrm{C} & -0.78130900 & 0.17456400 & -0.20118800 \\ \mathrm{C} & -0.75508800 & 1.66862900 & -0.54015300 \\ \mathrm{Si} & 0.78598400 & 2.46772500 & 0.16345700 \\ \mathrm{C} & 1.32544700 & 4.01058600 & -0.73762100 \\ \mathrm{C} & 0.62070400 & 2.78094900 & 2.00510800 \\ \mathrm{C} & 0.72849900 & -0.50767600 & -2.16902500 \\ \mathrm{C} & 0.24119700 & -2.05927500 & -0.29642800 \\ \mathrm{O} & 0.06881500 & -2.94617500 & -1.07983300 \\ \mathrm{O} & 0.15079700 & -2.23816600 & 1.01610200 \\ \mathrm{C} & -0.14126200 & -3.55638000 & 1.46920600 \\ \mathrm{H} & 1.55262600 & -0.22300600 & 1.17701700\end{array}$




$\begin{array}{lrrr}\mathrm{H} & -0.81995800 & 0.06095600 & 0.87697500 \\ \mathrm{H} & -1.65635600 & 2.13222000 & -0.15419200 \\ \mathrm{H} & -0.77289400 & 1.80433800 & -1.61828000 \\ \mathrm{H} & 2.27232500 & 4.37015900 & -0.34805700 \\ \mathrm{H} & 0.59309200 & 4.80302800 & -0.61786500 \\ \mathrm{H} & 1.44781800 & 3.81921500 & -1.79812600 \\ \mathrm{H} & -0.12678700 & 3.54468200 & 2.19779800 \\ \mathrm{H} & 1.56312600 & 3.12356700 & 2.42053100 \\ \mathrm{H} & 0.32386100 & 1.88638600 & 2.54299700 \\ \mathrm{H} & 1.50630700 & -1.18663700 & -2.49106600 \\ \mathrm{H} & 1.02502000 & 0.49618300 & -2.43963800 \\ \mathrm{H} & -0.16489000 & -0.76761700 & -2.72135500 \\ \mathrm{H} & -0.17302400 & -3.49559800 & 2.54558000 \\ \mathrm{H} & 0.63093000 & -4.24380100 & 1.15136000 \\ \mathrm{H} & -1.09527300 & -3.88740400 & 1.08200500 \\ \mathrm{C} & 3.11184800 & -0.72436400 & -0.20142700 \\ \mathrm{H} & 3.33591600 & -0.48062900 & -1.23613100 \\ \mathrm{C} & 3.17447700 & -2.23772900 & -0.03786800 \\ \mathrm{H} & 4.19529500 & -2.56955200 & -0.19223400 \\ \mathrm{H} & 2.55979800 & -2.77251400 & -0.74914300 \\ \mathrm{H} & 2.88480500 & -2.53940700 & 0.96389300 \\ \mathrm{C} & 4.19132100 & -0.08703800 & 0.66844600 \\ \mathrm{H} & 4.22031600 & 0.98578900 & 0.54459400 \\ \mathrm{H} & 5.16516100 & -0.48860600 & 0.40934300 \\ \mathrm{H} & 4.01472800 & -0.30491800 & 1.71907400 \\ \mathrm{C} & -2.04191500 & -0.47539500 & -0.76087300 \\ \mathrm{H} & -2.06736500 & -1.54669500 & -0.65652700 \\ \mathrm{H} & -2.21492800 & -0.21343700 & -1.79203200 \\ \mathrm{Br} & -3.66005800 & 0.12438000 & 0.18800700\end{array}$

\section{4}

$E=-3599.642754$ Hartree

$G=-3599.309738$ Hartree

qG (G with quasiharmonic correction $)=-3599.307342$ Hartree

$\mathrm{N}_{\text {(imaginary) }}=0$

$\begin{array}{lrrr}\mathrm{O} & -0.58492600 & 1.74551300 & 0.18475400 \\ \mathrm{C} & -1.10680600 & 0.61404400 & -0.46408500 \\ \mathrm{C} & -0.89933400 & -0.65782700 & 0.43171800 \\ \mathrm{C} & 0.49628100 & -1.32264100 & 0.20701100 \\ \mathrm{C} & 2.05397000 & 0.70132100 & 0.70881900 \\ \mathrm{Si} & 0.99935300 & 2.22802100 & 0.32185000 \\ \mathrm{C} & 1.05574300 & 3.47192300 & 1.71150500 \\ \mathrm{C} & 1.57783300 & 2.97835100 & -1.29555500 \\ \mathrm{C} & -1.07672900 & -0.34617400 & 1.91689500 \\ \mathrm{C} & -2.53959100 & 0.94964500 & -0.91014100 \\ \mathrm{C} & -1.91123300 & -1.69893400 & -0.04292400 \\ \mathrm{O} & -1.82236500 & -2.29138700 & -1.07979700 \\ \mathrm{O} & -2.92349900 & -1.87588400 & 0.79146600 \\ \mathrm{C} & -3.92748300 & -2.80492200 & 0.39415100 \\ \mathrm{H} & -0.56077600 & 0.42481400 & -1.38631500 \\ \mathrm{H} & 0.39299200 & -2.06230800 & -0.57289600 \\ \mathrm{H} & 0.74696700 & -1.85613200 & 1.11714100 \\ \mathrm{H} & 1.98567800 & 0.42124100 & 1.75454300 \\ \mathrm{H} & 3.08349600 & 0.99439300 & 0.53083400 \\ \mathrm{H} & 0.69956500 & 3.03705500 & 2.63899700 \\ \mathrm{H} & 0.43111800 & 4.32836400 & 1.47969400 \\ \mathrm{H} & 2.06841200 & 3.82881200 & 1.87071500 \\ \mathrm{H} & 2.60354300 & 3.32489700 & -1.21547900 \\ \mathrm{H} & 0.95634400 & 3.82779600 & -1.55983300\end{array}$




$\begin{array}{lrrr}\mathrm{H} & 1.53217800 & 2.26797000 & -2.11476100 \\ \mathrm{H} & -0.27762100 & 0.29217500 & 2.26335200 \\ \mathrm{H} & -1.04379700 & -1.26322700 & 2.49227400 \\ \mathrm{H} & -2.01064500 & 0.14983200 & 2.12848300 \\ \mathrm{H} & -2.95058200 & 0.05582700 & -1.36958500 \\ \mathrm{H} & -4.64673300 & -2.81903800 & 1.19777700 \\ \mathrm{H} & -3.49806200 & -3.78718600 & 0.25282800 \\ \mathrm{H} & -4.39600600 & -2.48463300 & -0.52666900 \\ \mathrm{C} & -2.48227800 & 2.01905000 & -1.99585000 \\ \mathrm{H} & -3.47382500 & 2.20881200 & -2.39200900 \\ \mathrm{H} & -1.84793600 & 1.71373200 & -2.82202400 \\ \mathrm{H} & -2.09634900 & 2.95100600 & -1.59888100 \\ \mathrm{C} & -3.46884700 & 1.39656700 & 0.21121300 \\ \mathrm{H} & -3.03866300 & 2.22975500 & 0.75423400 \\ \mathrm{H} & -3.68372300 & 0.60101500 & 0.91191800 \\ \mathrm{H} & -4.41423500 & 1.72412000 & -0.20875600 \\ \mathrm{C} & 1.69309400 & -0.46184200 & -0.18016400 \\ \mathrm{H} & 1.62156300 & -0.15522200 & -1.21253000 \\ \mathrm{Br} & 3.24813600 & -1.73724200 & -0.29273900\end{array}$

Bimolecular Additions (Table 2 of the manuscript)

\begin{tabular}{|c|c|}
\hline Structure & $\mathrm{G}_{\mathrm{rel}}\left(\mathrm{kcal} \cdot \mathrm{mol}^{-1}\right)$ \\
\hline TS D' & qG TS D' $-\mathrm{qG}$ TS B' $=4.44$ \\
\hline TS B' & 0 \\
\hline
\end{tabular}

\section{TS D'}

$E=-1101.837028$ Hartree

$\mathrm{G}=-1101.487410$ Hartree

qG (G with quasiharmonic correction $)=-1101.484733$ Hartree

$\mathrm{N}_{\text {(imaginary) }}=1, v=-614.2 \mathrm{i} \mathrm{cm}^{-1}$

$\begin{array}{lrrr}\mathrm{C} & 1.01649500 & -1.24389400 & -0.18047300 \\ \mathrm{C} & 1.11840400 & 0.13648700 & 0.45757200 \\ \mathrm{C} & -0.69155000 & 1.10956600 & -0.08498200 \\ \mathrm{C} & -1.75241200 & 0.60765200 & 0.86330400 \\ \mathrm{C} & -4.55171600 & -0.70130300 & 1.12233200 \\ \mathrm{C} & -4.05115400 & 1.30039300 & -1.16621300 \\ \mathrm{C} & -0.45734800 & 2.47190900 & -0.14222600 \\ \mathrm{C} & 1.17007300 & 0.17650600 & 1.95311000 \\ \mathrm{C} & 2.00336100 & 1.05321900 & -0.27828600 \\ \mathrm{O} & 2.16345600 & 1.04243600 & -1.47137700 \\ \mathrm{O} & 2.59614300 & 1.95430600 & 0.50627300 \\ \mathrm{C} & 3.41472700 & 2.91947600 & -0.13710100 \\ \mathrm{H} & 1.00413600 & -1.11089900 & -1.25843100 \\ \mathrm{H} & -0.61405000 & 0.59474200 & -1.02906900 \\ \mathrm{H} & -2.01996300 & 1.38577200 & 1.57156700 \\ \mathrm{H} & -1.41368500 & -0.24424900 & 1.43900600 \\ \mathrm{H} & -5.39414900 & -1.12481300 & 0.58388100 \\ \mathrm{H} & -4.93749400 & 0.07114000 & 1.78075700 \\ \mathrm{H} & -4.11785000 & -1.48293500 & 1.73688400 \\ \mathrm{H} & -4.45995600 & 2.11611600 & -0.57760500 \\ \mathrm{H} & -4.86304800 & 0.87908700 & -1.75173400 \\ \mathrm{H} & -3.31946100 & 1.71516800 & -1.85060700 \\ \mathrm{H} & -0.74151600 & 3.11832100 & 0.66939200 \\ \mathrm{H} & 0.10165000 & 2.90787700 & -0.94969900 \\ \mathrm{H} & 2.16627700 & -0.06863300 & 2.31350700 \\ \mathrm{H} & 0.48414100 & -0.54745000 & 2.37186700\end{array}$




$\begin{array}{lrrr}\mathrm{H} & 0.92458000 & 1.15872000 & 2.33499600 \\ \mathrm{H} & 3.79870800 & 3.55093900 & 0.64935000 \\ \mathrm{H} & 2.83605900 & 3.50570100 & -0.83887900 \\ \mathrm{H} & 4.22864000 & 2.43995500 & -0.66452700 \\ \mathrm{C} & 2.19469900 & -2.16918100 & 0.17458000 \\ \mathrm{H} & 2.09606400 & -2.39234300 & 1.23454200 \\ \mathrm{C} & 3.56838900 & -1.55749500 & -0.06247100 \\ \mathrm{H} & 4.33699900 & -2.28413100 & 0.17866100 \\ \mathrm{H} & 3.75188300 & -0.68408900 & 0.55306600 \\ \mathrm{H} & 3.69752000 & -1.26867700 & -1.09996700 \\ \mathrm{C} & 2.07238200 & -3.47862100 & -0.59420800 \\ \mathrm{H} & 1.11315100 & -3.94734500 & -0.42231700 \\ \mathrm{H} & 2.85110800 & -4.17096600 & -0.29061800 \\ \mathrm{H} & 2.18275700 & -3.30677300 & -1.66210200 \\ \mathrm{O} & -0.14736900 & -1.92482000 & 0.23928600 \\ \mathrm{H} & -0.89100600 & -1.72736300 & -0.33225600 \\ \mathrm{Si} & -3.27996000 & 0.00523700 & -0.05633300 \\ \mathrm{O} & -2.61838100 & -1.22556500 & -1.00054400 \\ \mathrm{H} & -3.15894600 & -1.67532600 & -1.63973000\end{array}$

\section{TS B'}

$E=-1101.845248$ Hartree

$\mathrm{G}=-1101.494687$ Hartree

qG (G with quasiharmonic correction) $=-1101.491815$ Hartree

$\mathrm{N}_{\text {(imaginary) }}=1, v=-574.8 \mathrm{i} \mathrm{cm}^{-1}$

$\begin{array}{lrrr}\mathrm{C} & -1.14345500 & 1.13257100 & -0.48532100 \\ \mathrm{C} & -1.25362200 & -0.26037300 & 0.10329000 \\ \mathrm{C} & -0.11425700 & -1.60696400 & -1.11830300 \\ \mathrm{C} & 1.21498000 & -1.30087500 & -1.30099300 \\ \mathrm{C} & 2.31300300 & -1.61764000 & -0.35483200 \\ \mathrm{C} & 4.61592700 & -0.74061600 & 1.48931700 \\ \mathrm{C} & 4.26831800 & 0.56293100 & -1.29052500 \\ \mathrm{C} & -0.68385700 & -0.46883400 & 1.47398300 \\ \mathrm{C} & -1.74629100 & 2.23301600 & 0.39923200 \\ \mathrm{C} & -2.51653000 & -0.93779900 & -0.22375800 \\ \mathrm{O} & -3.19189200 & -0.70787900 & -1.19435500 \\ \mathrm{O} & -2.82920500 & -1.90438400 & 0.64158700 \\ \mathrm{C} & -4.00452300 & -2.65362900 & 0.36301900 \\ \mathrm{H} & -1.66293600 & 1.13921200 & -1.43728500 \\ \mathrm{H} & -0.76655200 & -1.51882100 & -1.96945400 \\ \mathrm{H} & -0.35637400 & -2.39679300 & -0.42800500 \\ \mathrm{H} & 1.49133500 & -0.77344100 & -2.20334600 \\ \mathrm{H} & 1.92814600 & -2.13790100 & 0.51707800 \\ \mathrm{H} & 3.01895100 & -2.29742100 & -0.83774400 \\ \mathrm{H} & 4.10414400 & -1.14527400 & 2.35616300 \\ \mathrm{H} & 5.24141200 & 0.08038800 & 1.82437800 \\ \mathrm{H} & 5.26265200 & -1.51323100 & 1.08582100 \\ \mathrm{H} & 4.89813900 & -0.18205700 & -1.76745300 \\ \mathrm{H} & 4.89892200 & 1.39519200 & -0.99459800 \\ \mathrm{H} & 3.56938800 & 0.93205100 & -2.03493200 \\ \mathrm{H} & 0.30004000 & -0.02867000 & 1.55402900 \\ \mathrm{H} & -0.61377900 & -1.51947500 & 1.71921600 \\ \mathrm{H} & -1.31396400 & -0.00810400 & 2.23134300 \\ \mathrm{H} & -1.13583700 & 2.28205700 & 1.29798500 \\ \mathrm{H} & -4.08821900 & -3.37523100 & 1.16089500 \\ \mathrm{H} & -3.91977700 & -3.15625400 & -0.59125300 \\ \mathrm{H} & -4.87262800 & -2.00836500 & 0.34753300 \\ \mathrm{C} & -3.18516100 & 1.94729000 & 0.80635500 \\ \mathrm{H} & -3.56756100 & 2.77164100 & 1.39801700\end{array}$




$\begin{array}{lrrr}\mathrm{H} & -3.28049100 & 1.04971600 & 1.40665400 \\ \mathrm{H} & -3.82365600 & 1.83604200 & -0.06348300 \\ \mathrm{C} & -1.66091600 & 3.58042300 & -0.30620000 \\ \mathrm{H} & -2.26465400 & 3.57808700 & -1.21003600 \\ \mathrm{H} & -0.64291200 & 3.82240800 & -0.57924100 \\ \mathrm{H} & -2.03703100 & 4.36729500 & 0.33905800 \\ \mathrm{O} & 0.21562800 & 1.50524600 & -0.69809600 \\ \mathrm{H} & 0.54073200 & 1.00943800 & -1.44258700 \\ \mathrm{Si} & 3.38927700 & -0.14804700 & 0.20981200 \\ \mathrm{O} & 2.47143400 & 1.02150600 & 0.94735400 \\ \mathrm{H} & 1.69321200 & 1.33725200 & 0.48036700\end{array}$


Data File D: \RESULTS $\backslash 2018 K 23 \backslash 2018 K 29-2$ 2018-11-29 14-40-10 \AM-ESP-260_WHELK01_5IPA.D

Sample Name: AM-ESP-260
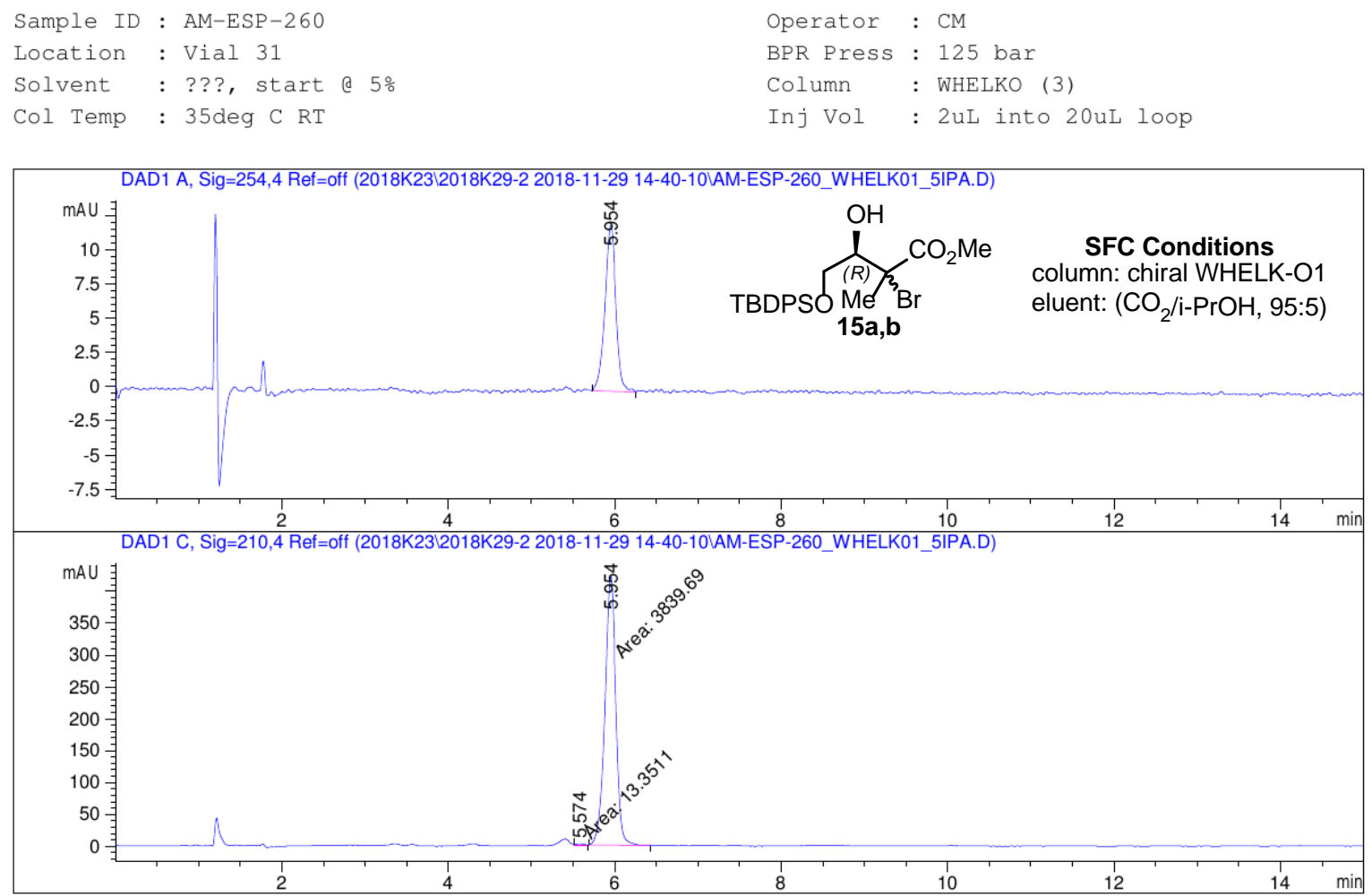

Area Percent Report

$\begin{array}{lll} & =================================== \\ & & \\ \text { Sorted By } & \text { Signal } & \\ \text { Multiplier: } & : & 1.0000 \\ \text { Dilution: } & : & 1.0000\end{array}$

Use Multiplier \& Dilution Factor with ISTDs

Signal 1: DAD1 A, Sig=254, 4 Ref=off

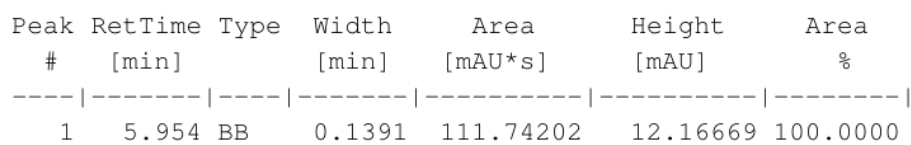

$\begin{array}{lll}\text { Totals : } & 111.74202 & 12.16669\end{array}$

Signal 2: DAD1 C, Sig=210, 4 Ref=off

\begin{tabular}{|c|c|c|c|c|c|c|}
\hline $\begin{array}{c}\text { Peak } \\
\#\end{array}$ & $\begin{array}{c}\text { RetTime } \\
\text { [min] }\end{array}$ & Type & $\begin{array}{l}\text { Width } \\
\text { [min] }\end{array}$ & $\begin{array}{c}\text { Area } \\
{\left[\mathrm{mAU}^{\star} \mathrm{S}\right]}\end{array}$ & $\begin{array}{l}\text { Height } \\
\text { [mAU] }\end{array}$ & $\begin{array}{c}\text { Area } \\
\frac{\%}{6}\end{array}$ \\
\hline & & & & & & \\
\hline 2 & 5.954 & MM & 0.1518 & 3839.69019 & 421.44434 & 99.6535 \\
\hline
\end{tabular}




\section{$\underline{\text { Part IV. }{ }^{1} \mathrm{H} \text { and }{ }^{13} \mathrm{C} \text { spectra }}$}

${ }^{1} \mathrm{H}-\mathrm{NMR}\left(500 \mathrm{MHz}, \mathrm{CDCl}_{3}\right)$

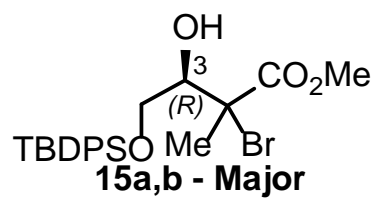

$$
\text { 15a,b - Major }
$$

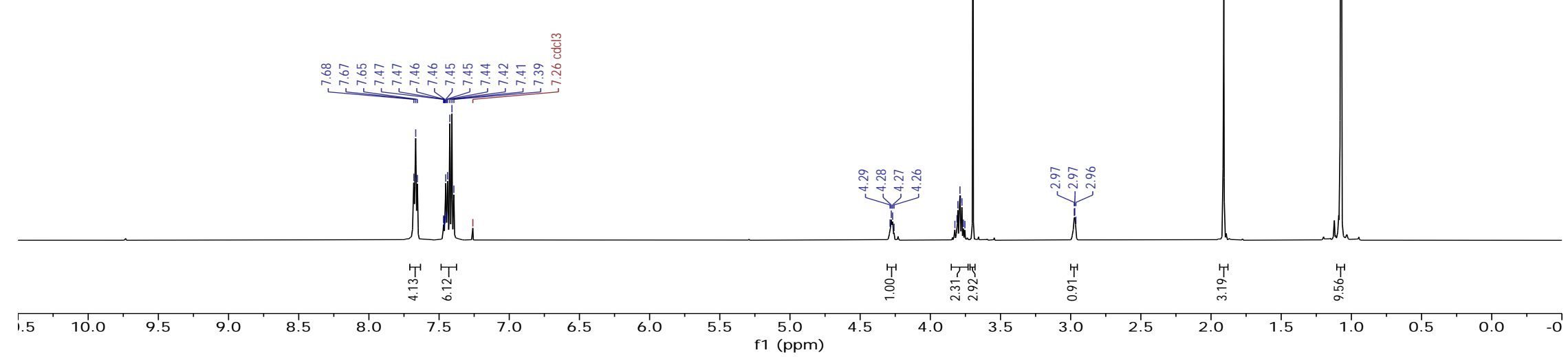


${ }^{1} \mathrm{H}-\mathrm{NMR}\left(500 \mathrm{MHz}, \mathrm{CDCl}_{3}\right)$

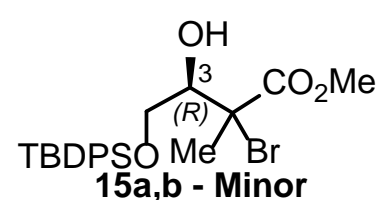

15a,b - Minor

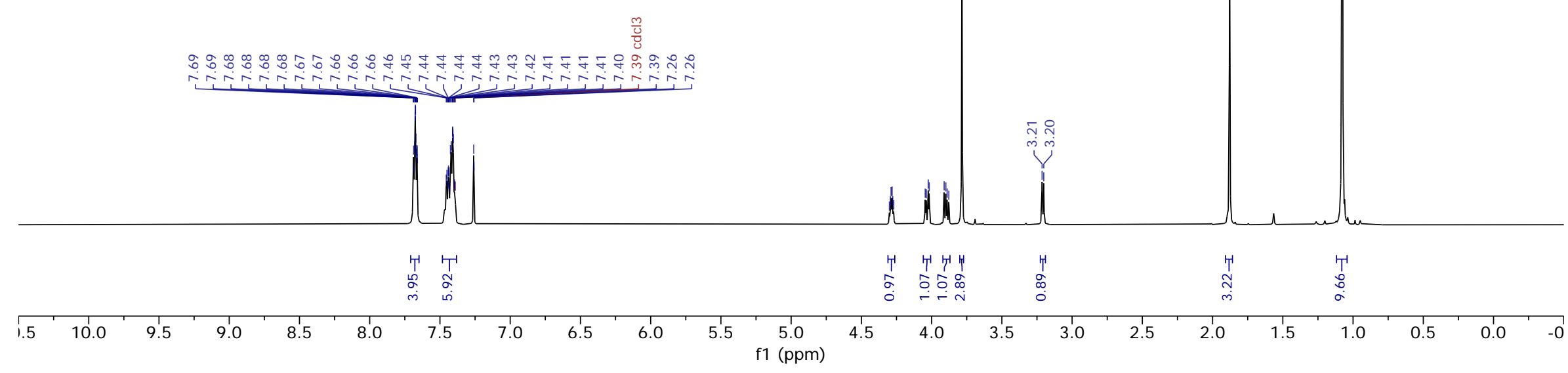


${ }^{1} \mathrm{H}-\mathrm{NMR}\left(500 \mathrm{MHz}, \mathrm{CDCl}_{3}\right.$ )<smiles>C=CC[Si](C)(C)O[C@@H](CC(=O)OCCCC)C(Br)(C(=O)OC)C(=O)OC</smiles>

17a,b-Major

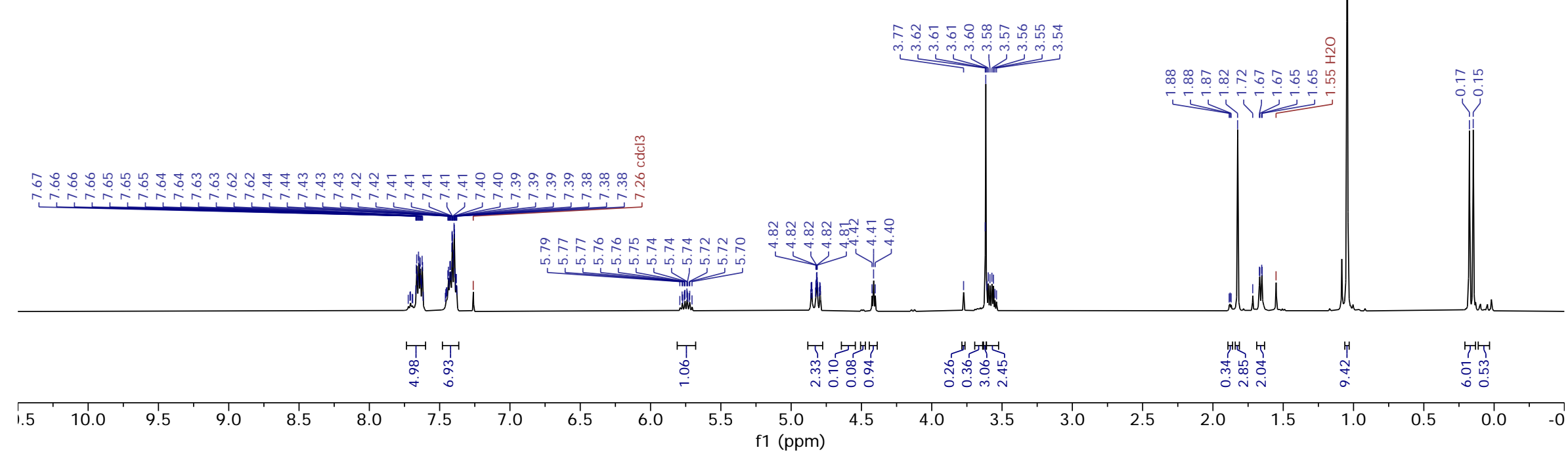


${ }^{1} \mathrm{H}-\mathrm{NMR}\left(500 \mathrm{MHz}, \mathrm{CDCl}_{3}\right.$ )

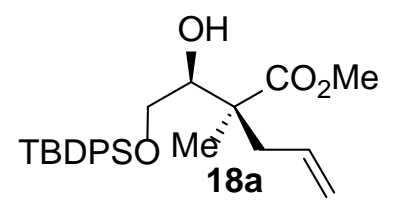

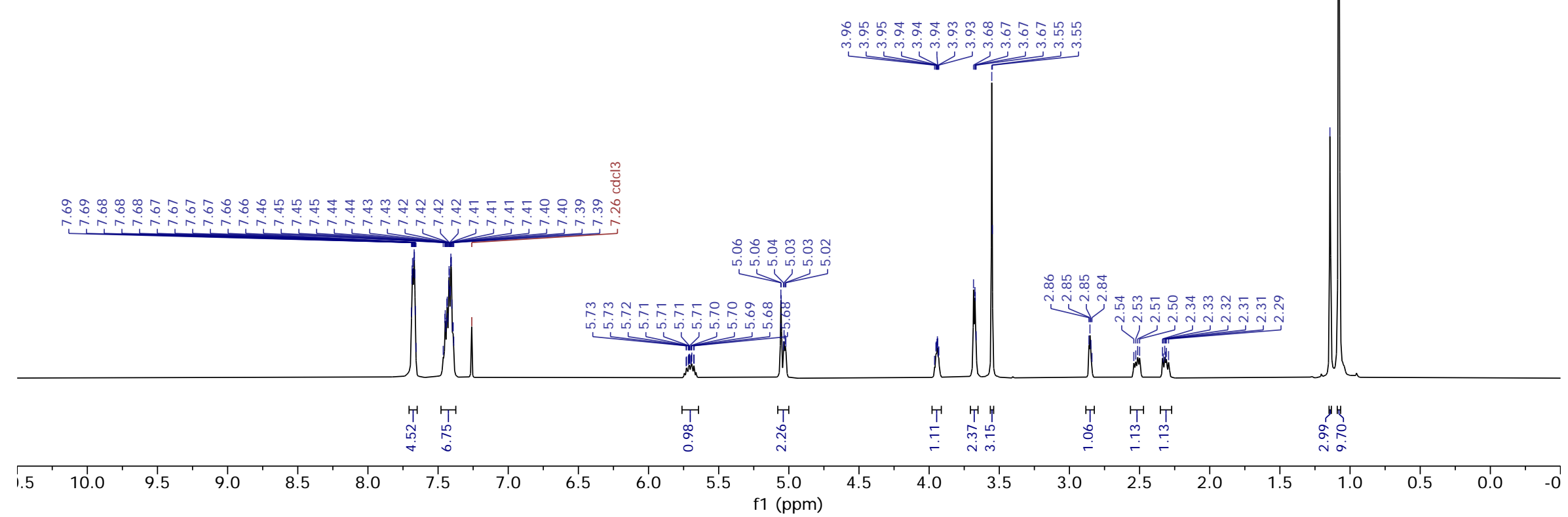


${ }^{1} \mathrm{H}-\mathrm{NMR}\left(500 \mathrm{MHz}, \mathrm{CDCl}_{3}\right)$
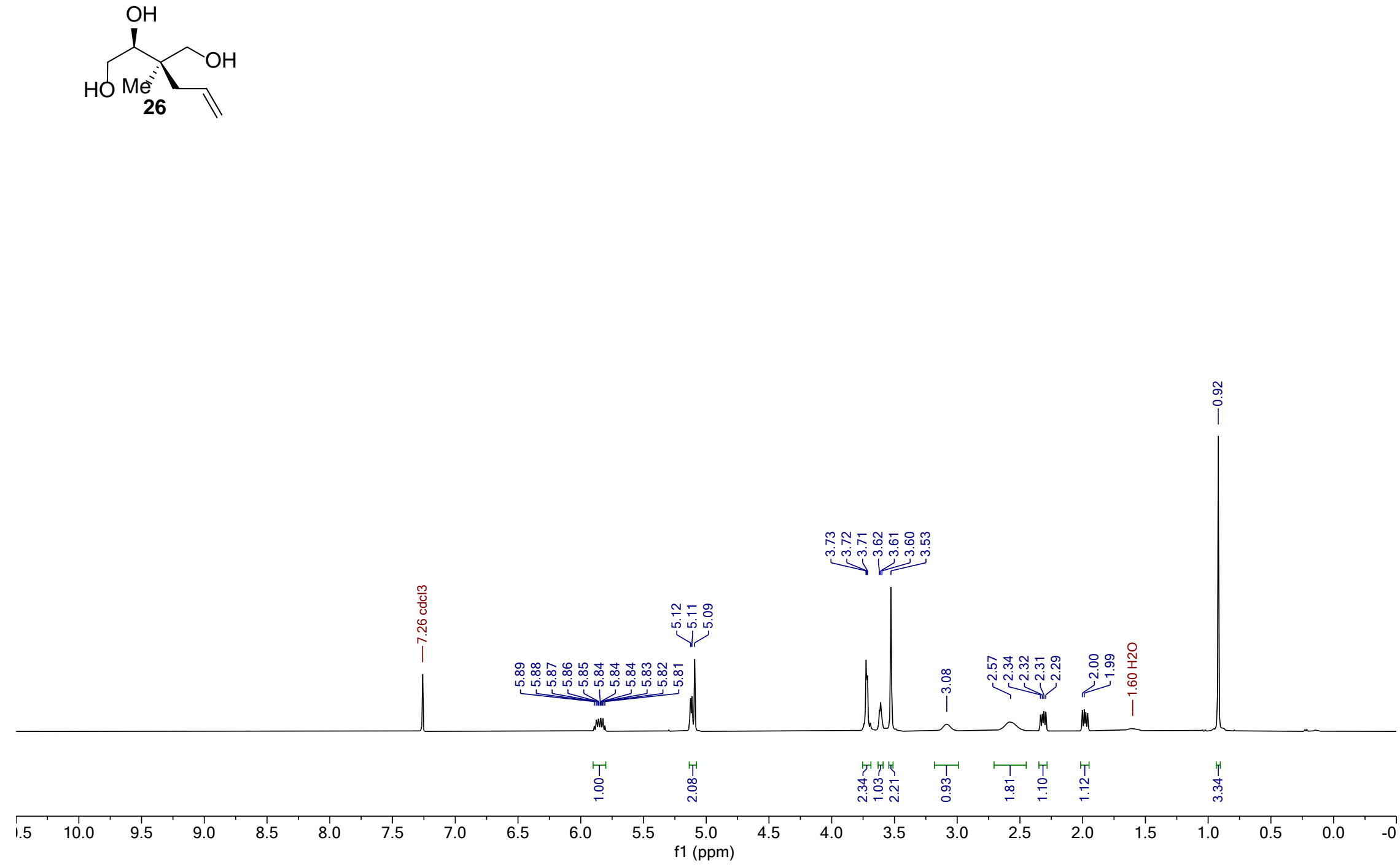
${ }^{1} \mathrm{H}-\mathrm{NMR}\left(500 \mathrm{MHz}, \mathrm{CDCl}_{3}\right.$ )

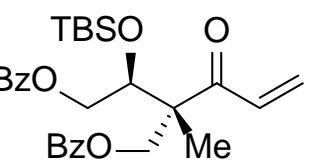

29

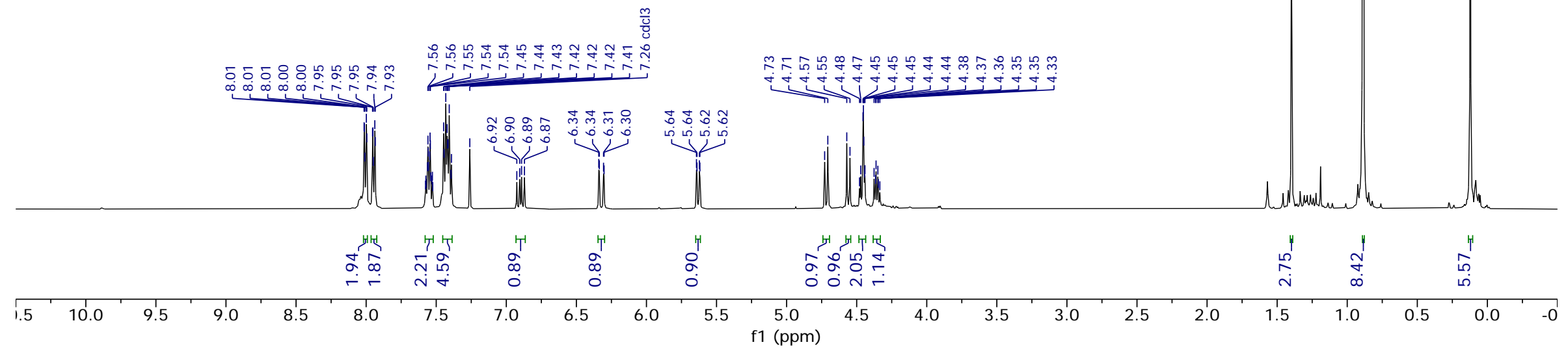


${ }^{13} \mathrm{C}\left\{{ }^{1} \mathrm{H}\right\}$-NMR (126 MHz, CDCl 3 )

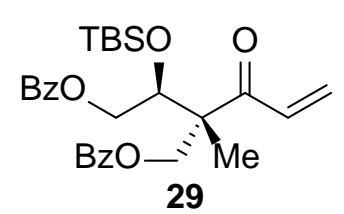

29

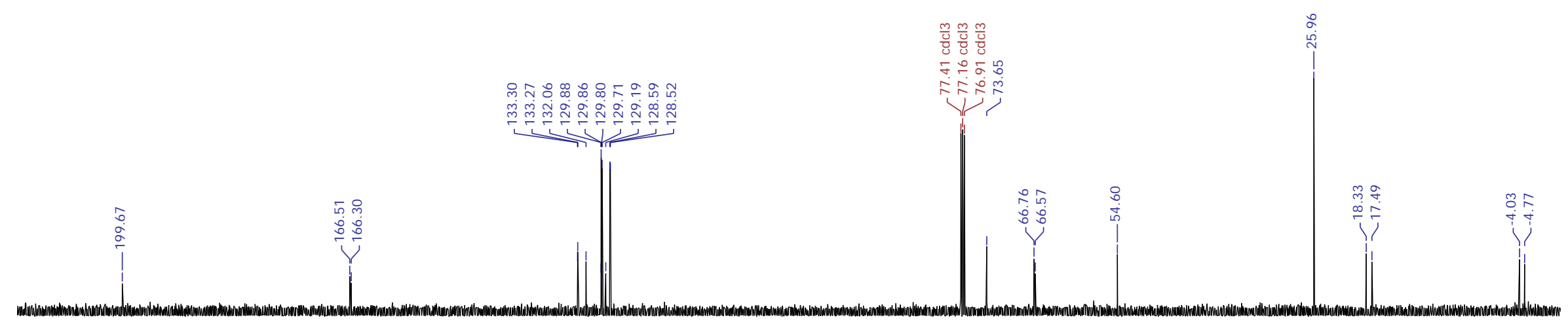

$210 \quad 200$

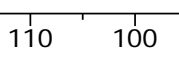


${ }^{1} \mathrm{H}-\mathrm{NMR}\left(500 \mathrm{MHz}, \mathrm{CDCl}_{3}\right)$

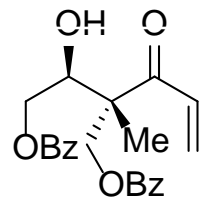

30

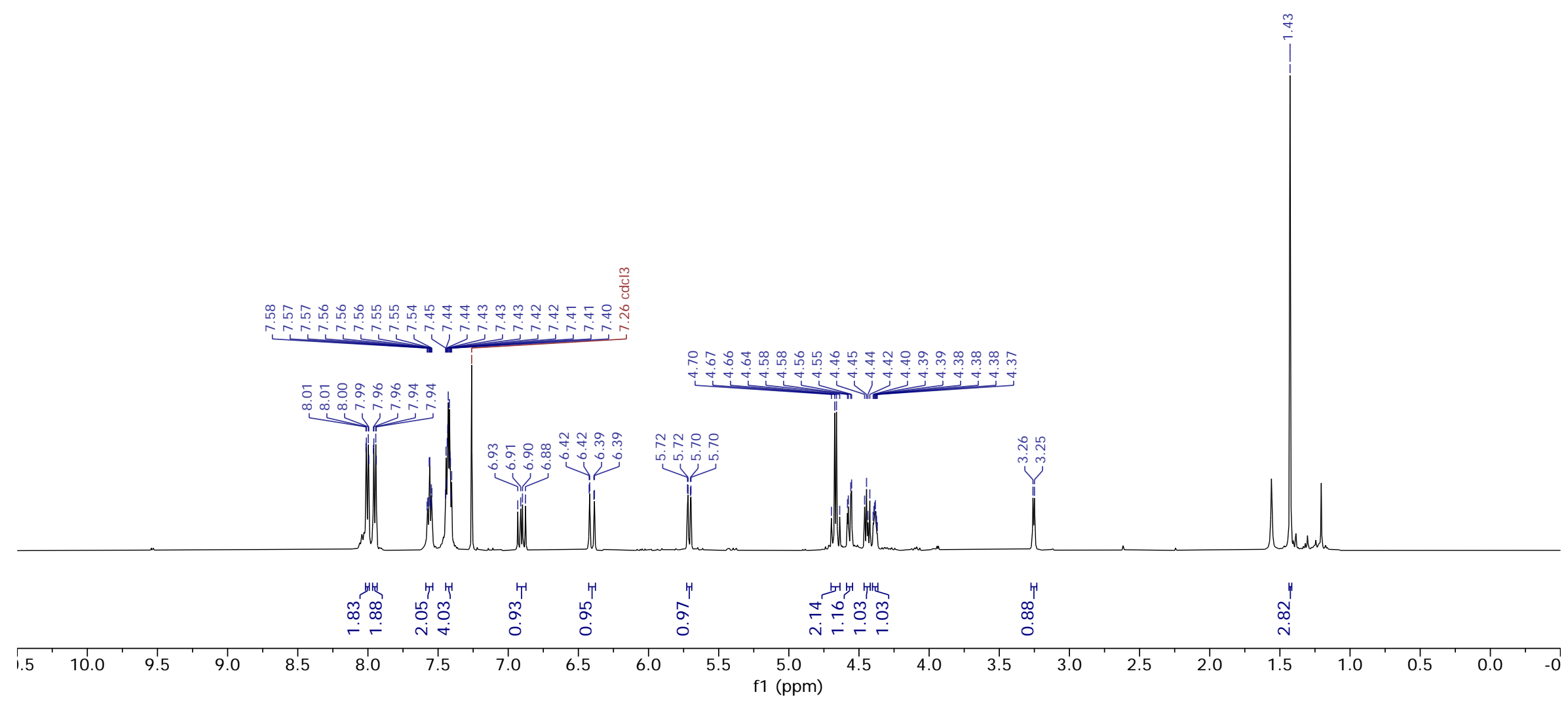


${ }^{13} \mathrm{C}\left\{{ }^{1} \mathrm{H}\right\}-\mathrm{NMR}\left(126 \mathrm{MHz}, \mathrm{CDCl}_{3}\right)$

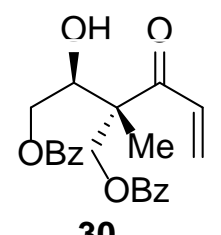

30

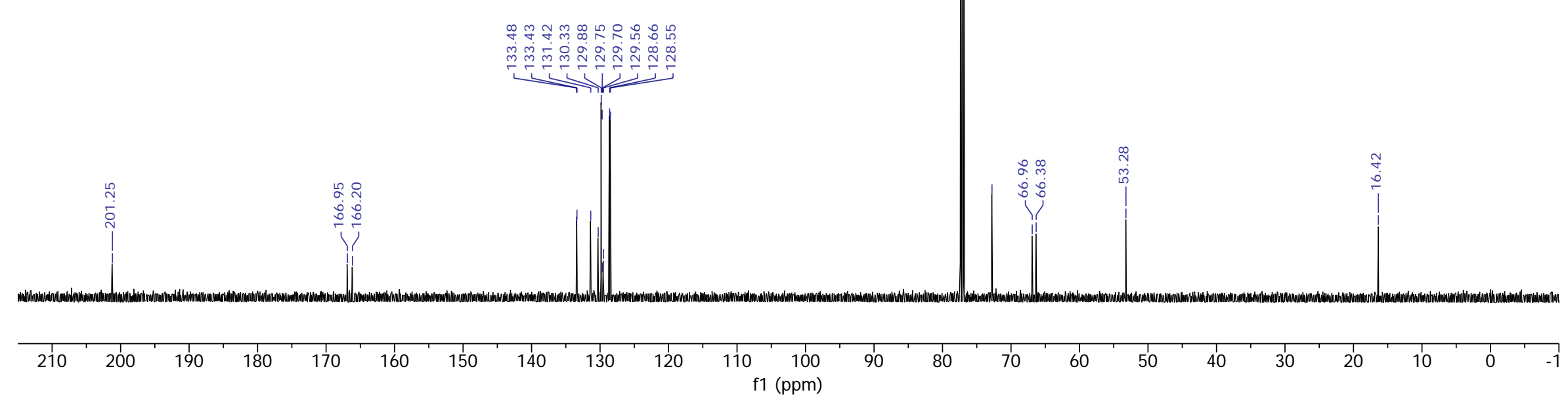


${ }^{1} \mathrm{H}-\mathrm{NMR}\left(500 \mathrm{MHz}, \mathrm{CDCl}_{3}\right)$
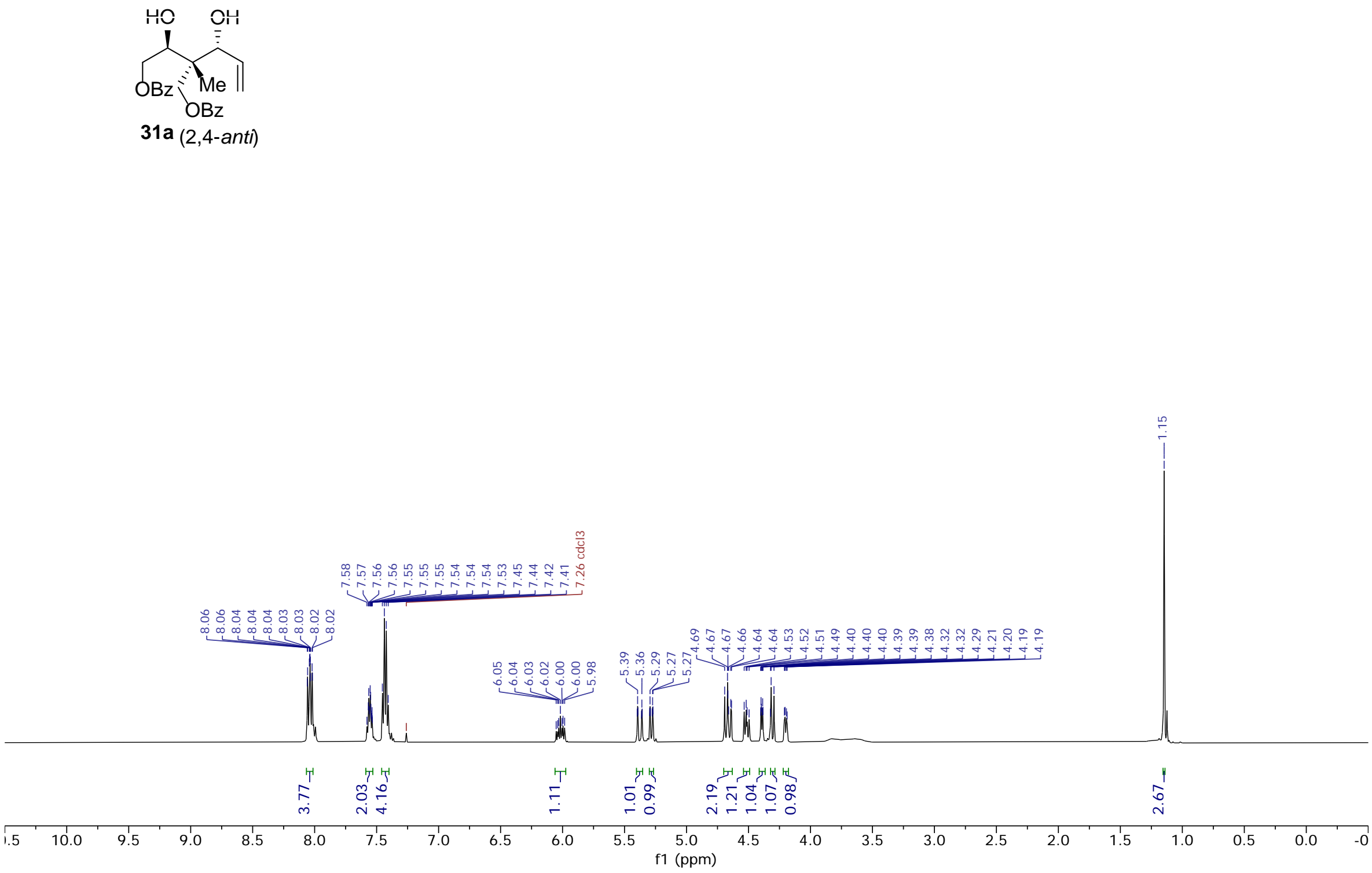
${ }^{13} \mathrm{C}\left\{{ }^{1} \mathrm{H}\right\}-\mathrm{NMR}\left(126 \mathrm{MHz}, \mathrm{CDCl}_{3}\right)$
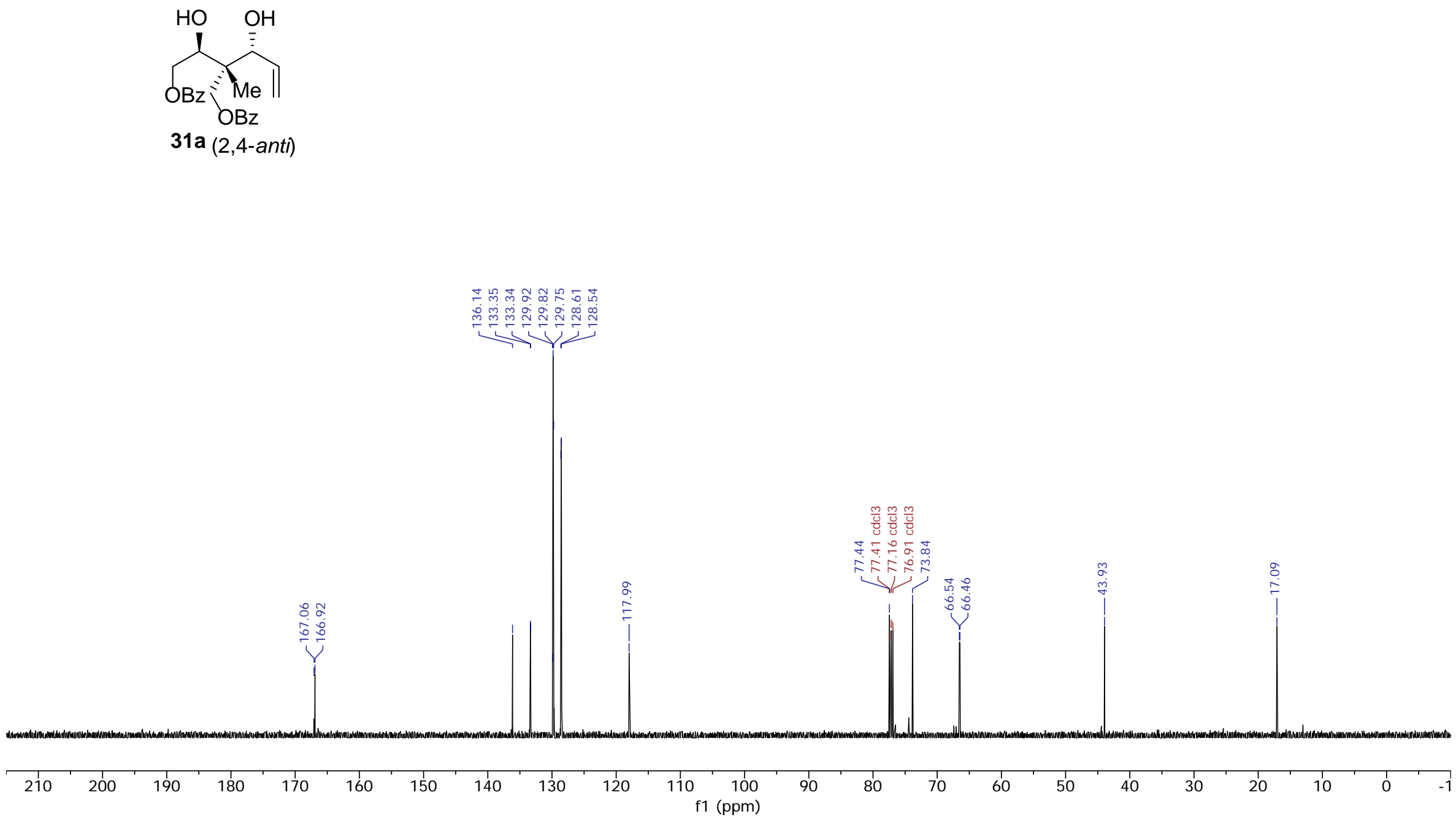
${ }^{1} \mathrm{H}-\mathrm{NMR}\left(500 \mathrm{MHz}, \mathrm{CDCl}_{3}\right)$

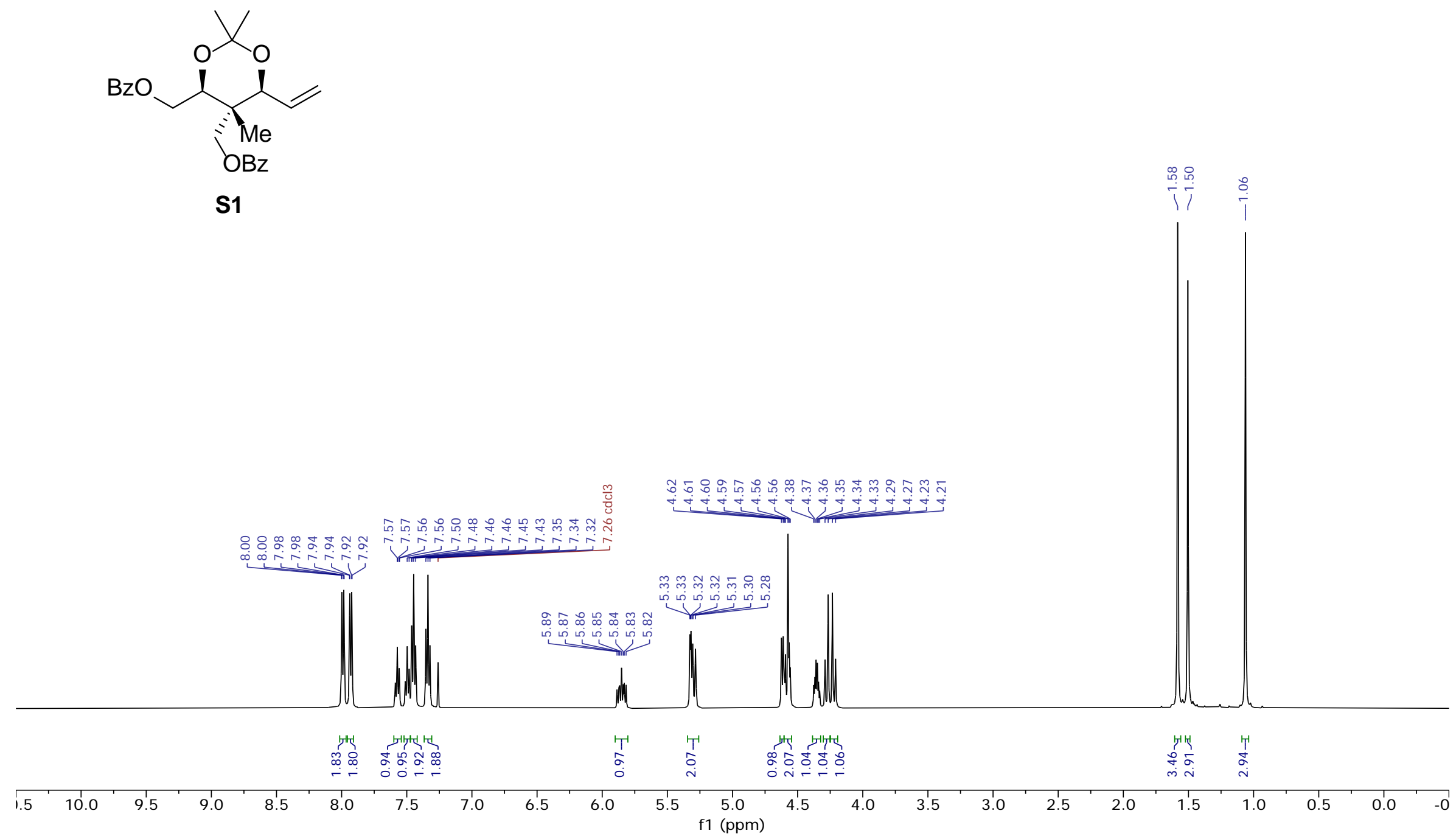


${ }^{13} \mathrm{C}\left\{{ }^{1} \mathrm{H}\right\}$-NMR $\left(126 \mathrm{MHz}, \mathrm{CDCl}_{3}\right)$

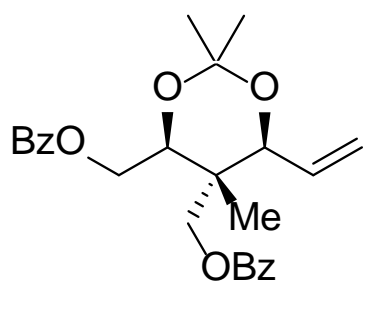

S1

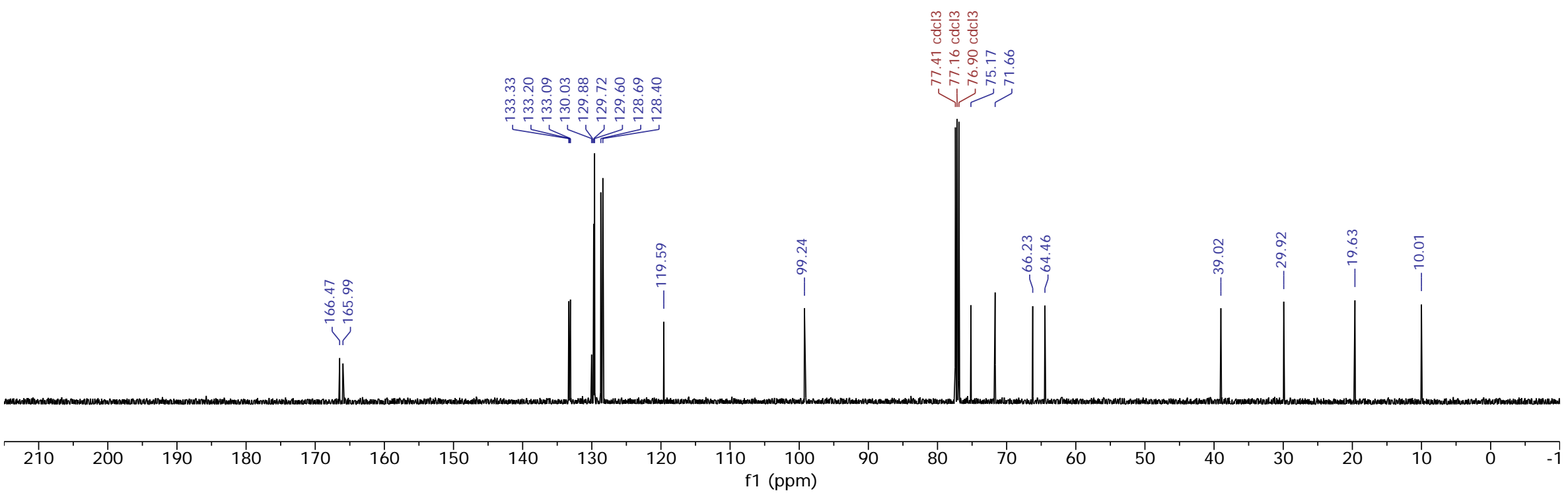


${ }^{1} \mathrm{H}-\mathrm{NMR}\left(500 \mathrm{MHz}, \mathrm{CDCl}_{3}\right)$

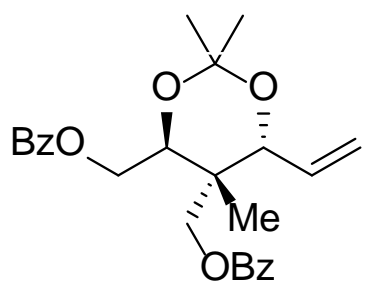

S2

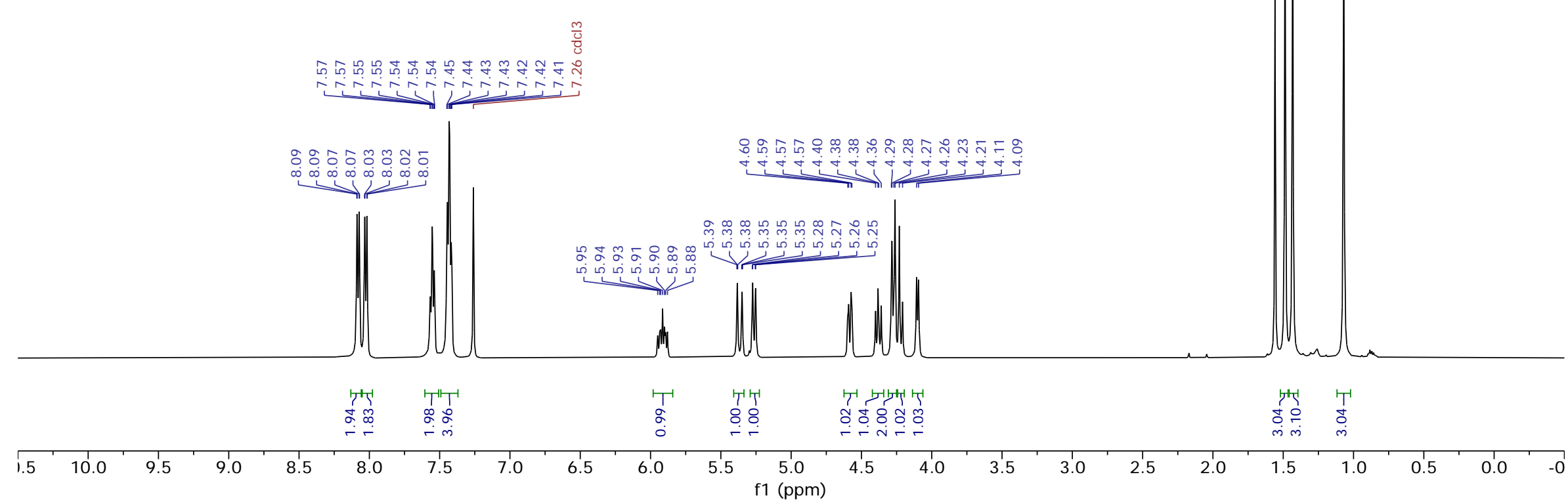


${ }^{13} \mathrm{C}\left\{{ }^{1} \mathrm{H}\right\}$-NMR $\left(126 \mathrm{MHz}, \mathrm{CDCl}_{3}\right)$

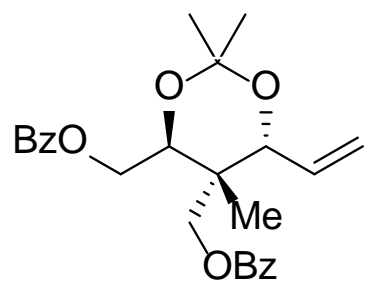

S2

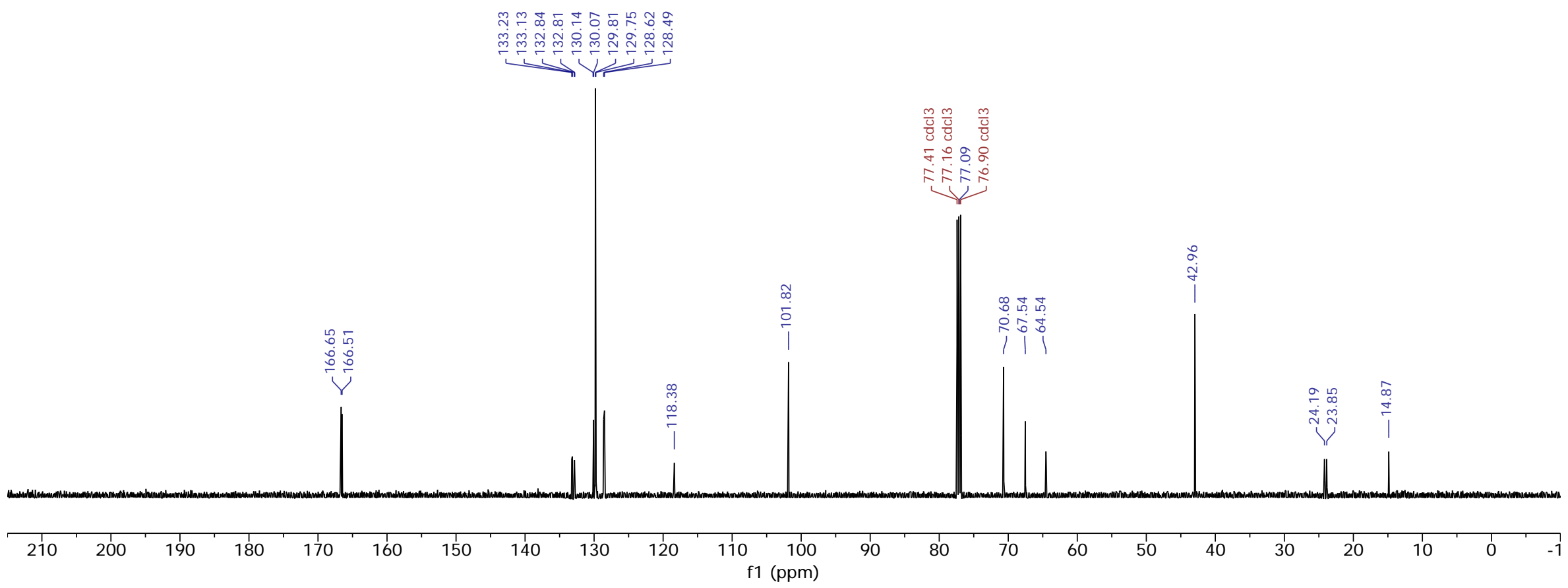


${ }^{1} \mathrm{H}-\mathrm{NMR}\left(500 \mathrm{MHz}, \mathrm{CDCl}_{3}\right)$

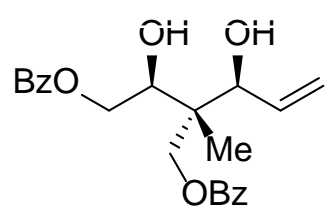

31b

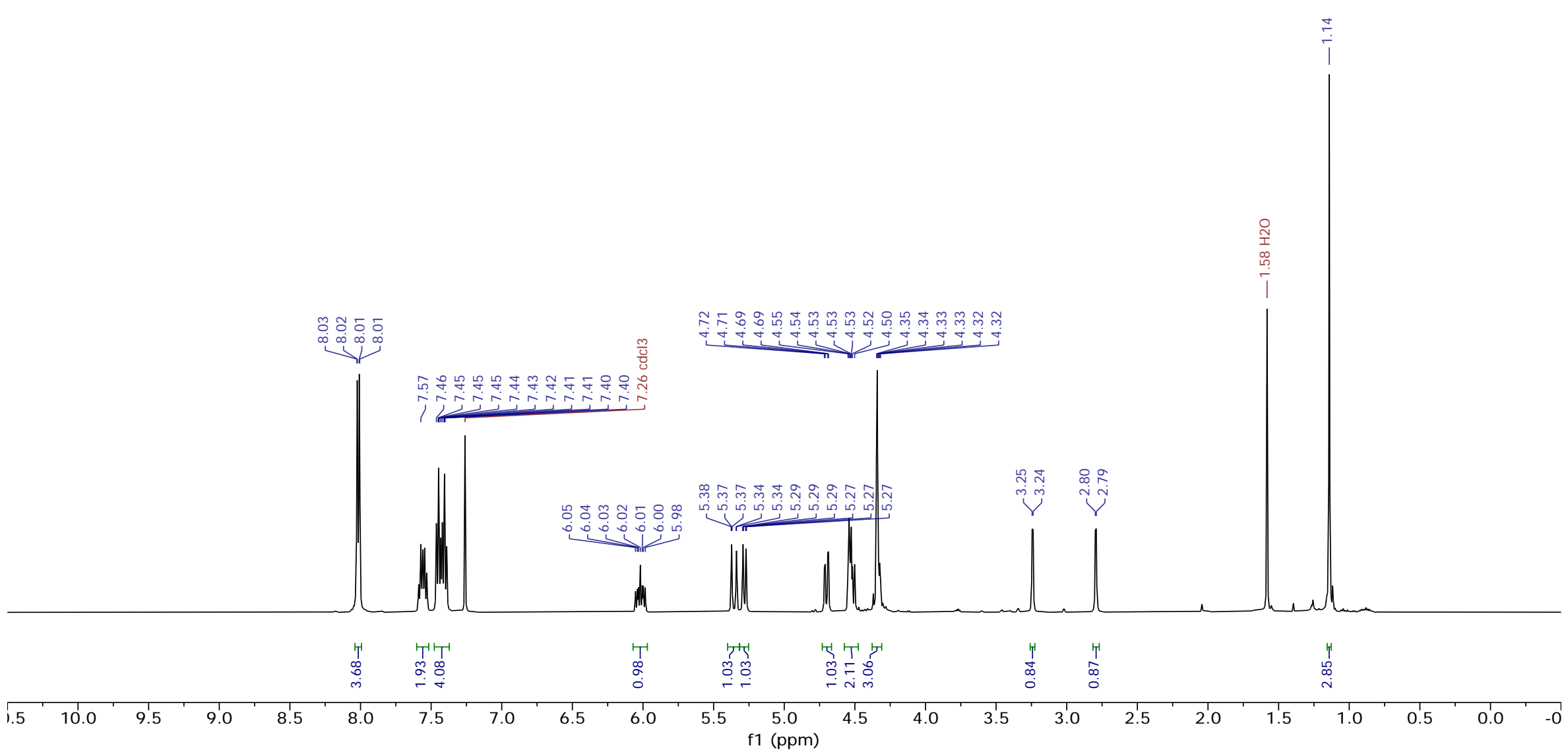


${ }^{13} \mathrm{C}\left\{{ }^{1} \mathrm{H}\right\}-\mathrm{NMR}\left(126 \mathrm{MHz}, \mathrm{CDCl}_{3}\right)$

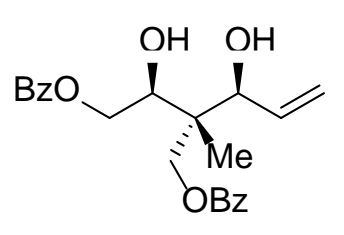

31b

$\frac{m}{0} \frac{m}{0} \frac{m}{0} \frac{m}{0}$

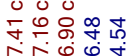

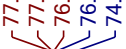

|

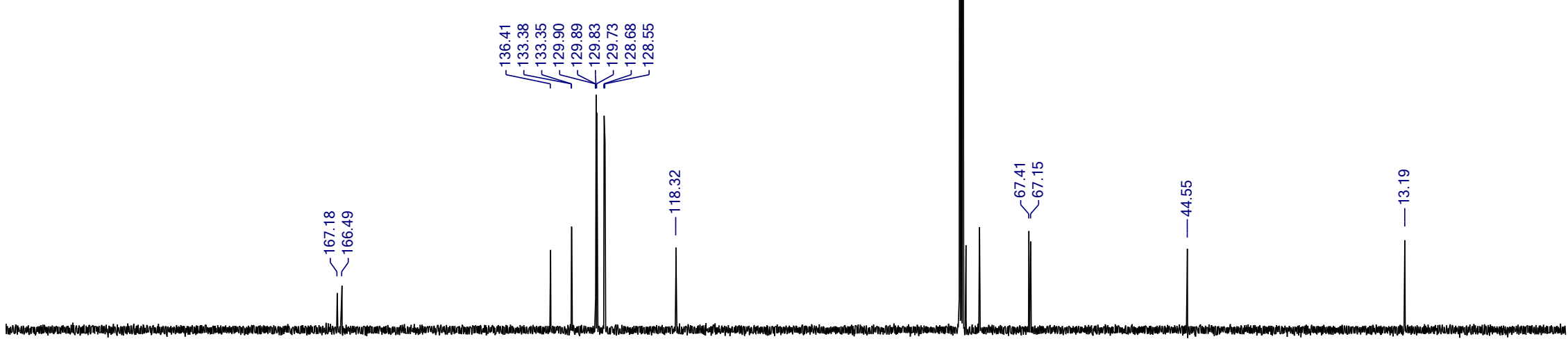

$\begin{array}{llllllll}210 & 200 & 190 & 180 & 170 & 160 & 150 & 140\end{array}$ $130 \quad 120$ $110 \quad 100$ $90 \quad 80$ $70 \quad 60$ $50 \quad 40$ 
${ }^{1} \mathrm{H}-\mathrm{NMR}\left(500 \mathrm{MHz}, \mathrm{CDCl}_{3}\right)$
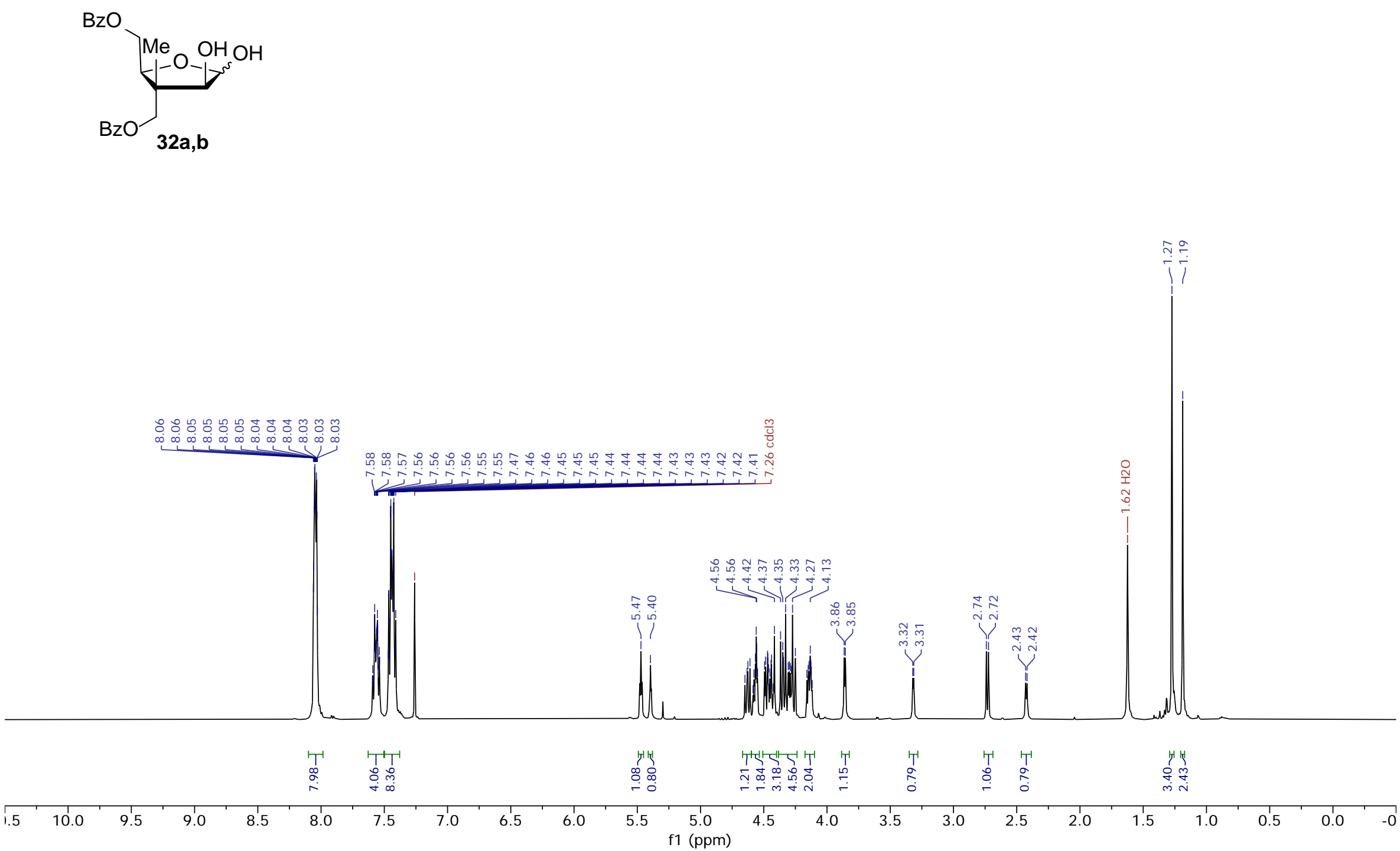
${ }^{13} \mathrm{C}\left\{{ }^{1} \mathrm{H}\right\}-\mathrm{NMR}\left(126 \mathrm{MHz}, \mathrm{CDCl}_{3}\right)$

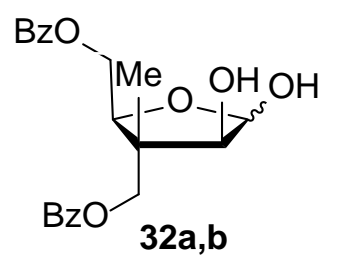

$32 a, b$
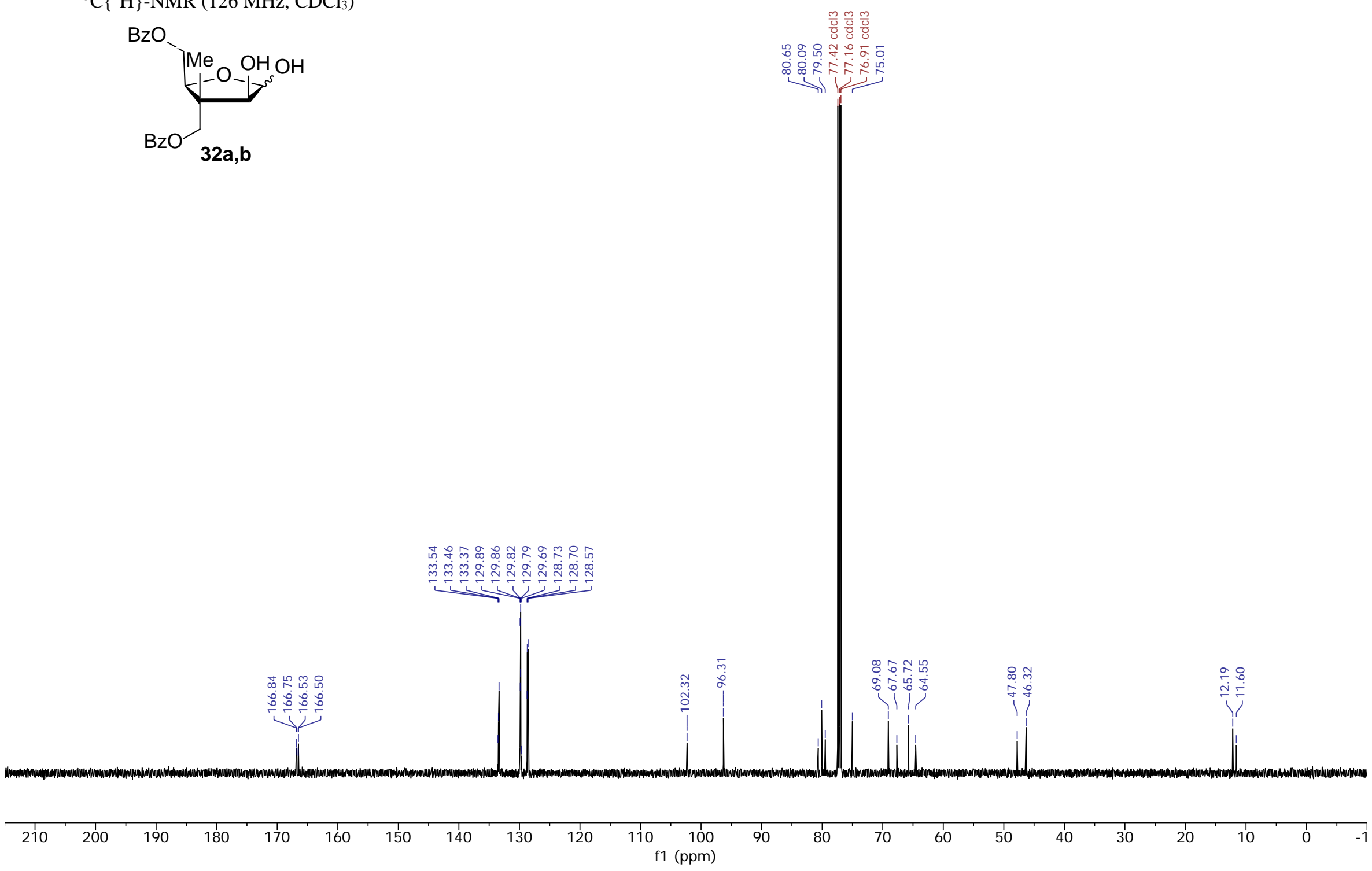
${ }^{1} \mathrm{H}-\mathrm{NMR}\left(500 \mathrm{MHz}, \mathrm{CDCl}_{3}\right)$
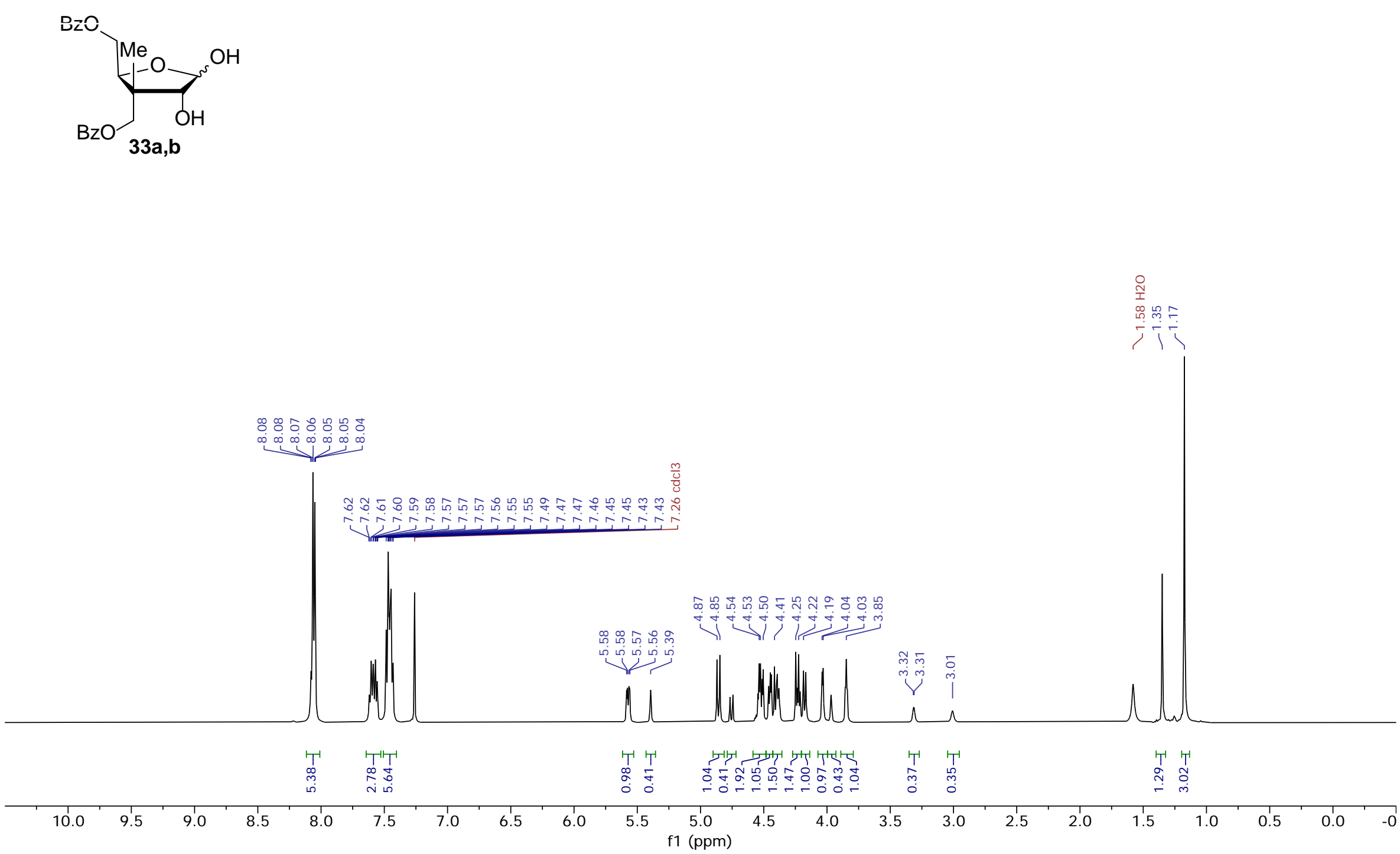
${ }^{13} \mathrm{C}\left\{{ }^{1} \mathrm{H}\right\}-\mathrm{NMR}\left(126 \mathrm{MHz}, \mathrm{CDCl}_{3}\right)$
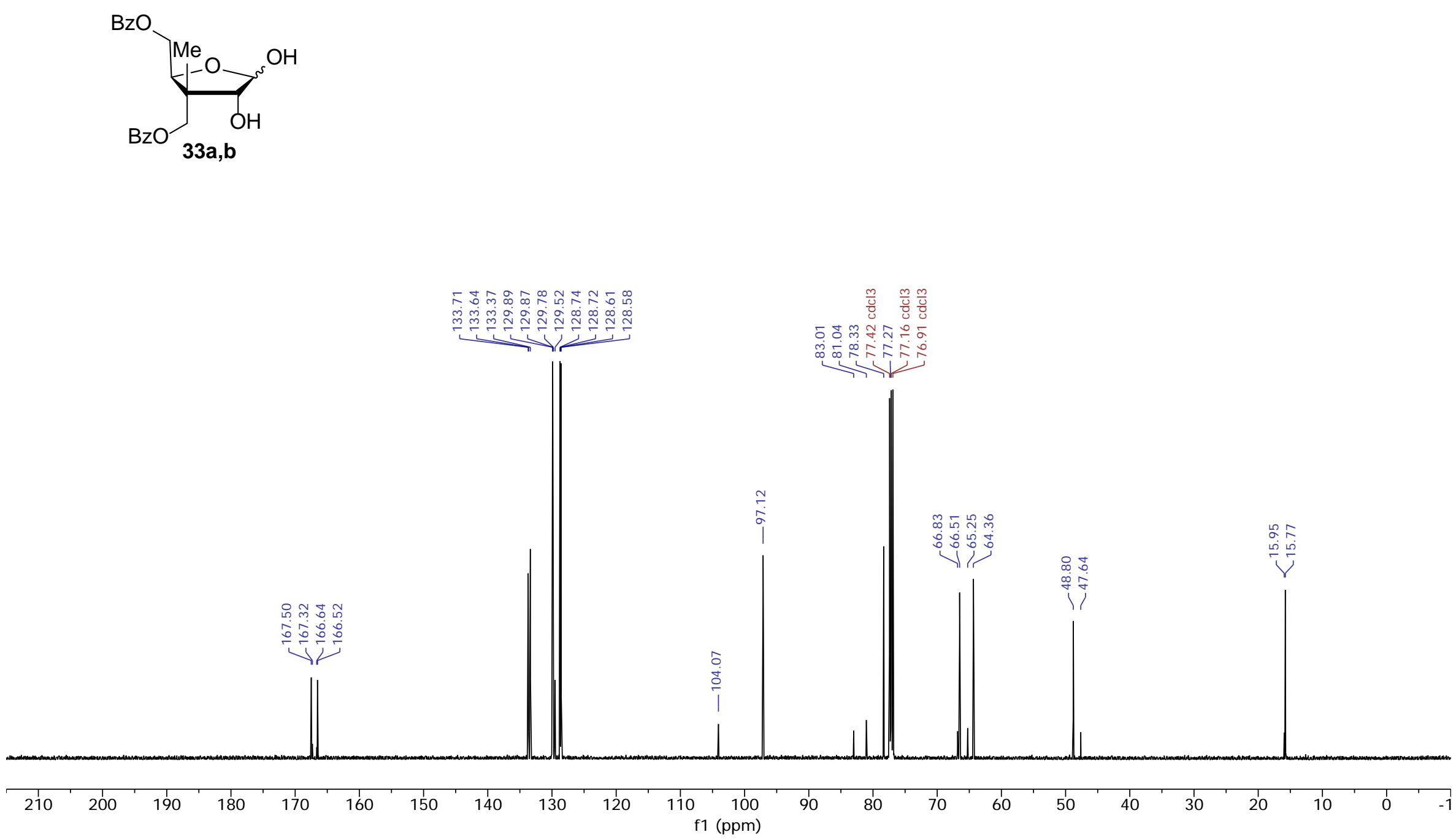
${ }^{1} \mathrm{H}-\mathrm{NMR}\left(500 \mathrm{MHz}, \mathrm{CD}_{3} \mathrm{OD}\right)$
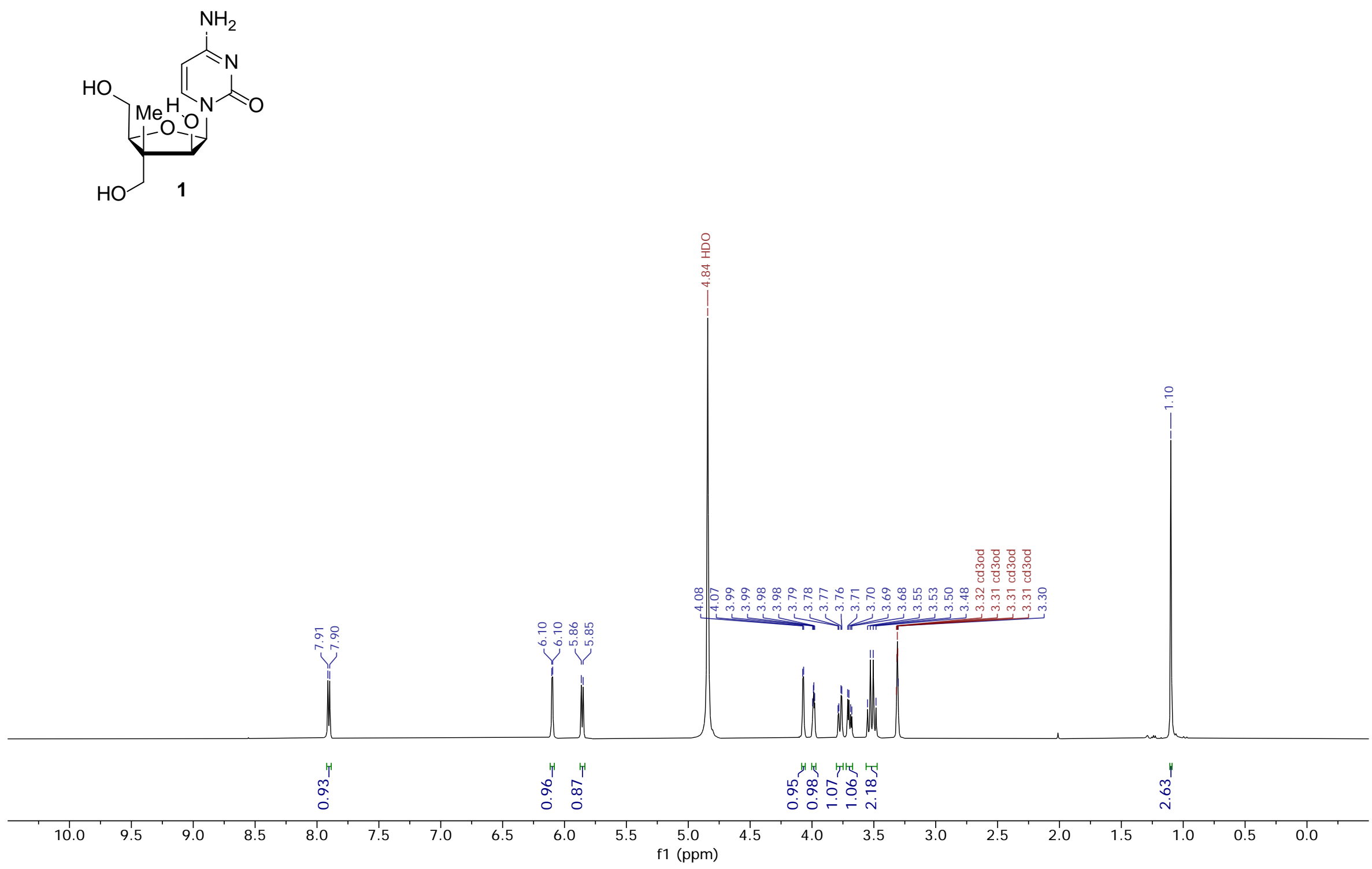
${ }^{13} \mathrm{C}\left\{{ }^{1} \mathrm{H}\right\}-\mathrm{NMR}\left(126 \mathrm{MHz}, \mathrm{CD}_{3} \mathrm{OD}\right)$
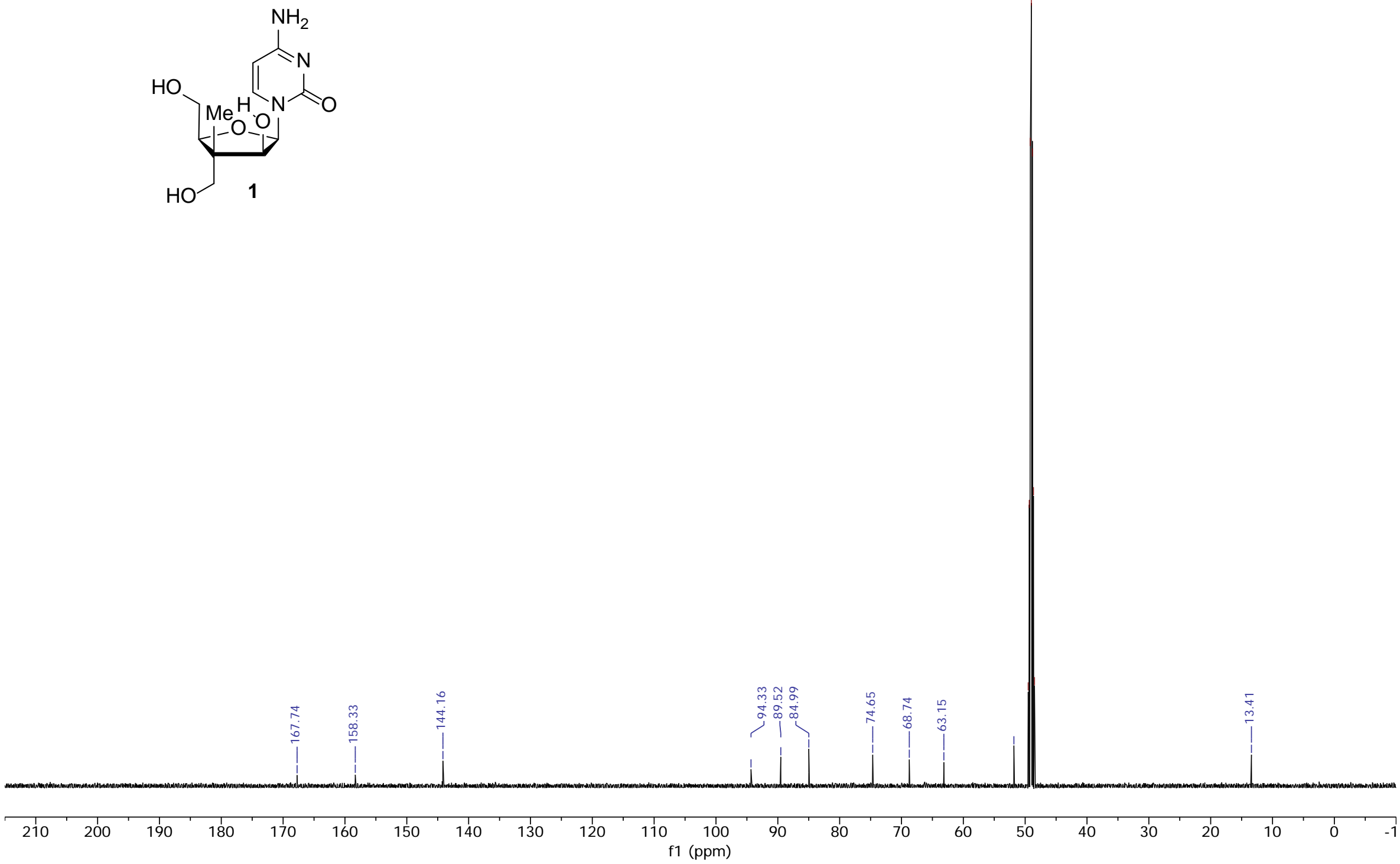
${ }^{1} \mathrm{H}-\mathrm{NMR}\left(500 \mathrm{MHz}, \mathrm{CDCl}_{3}\right)$

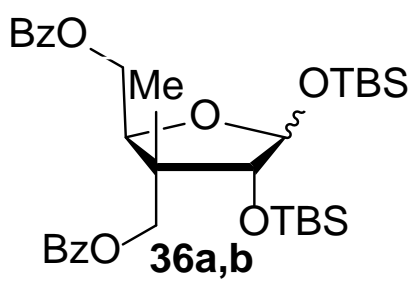

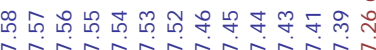

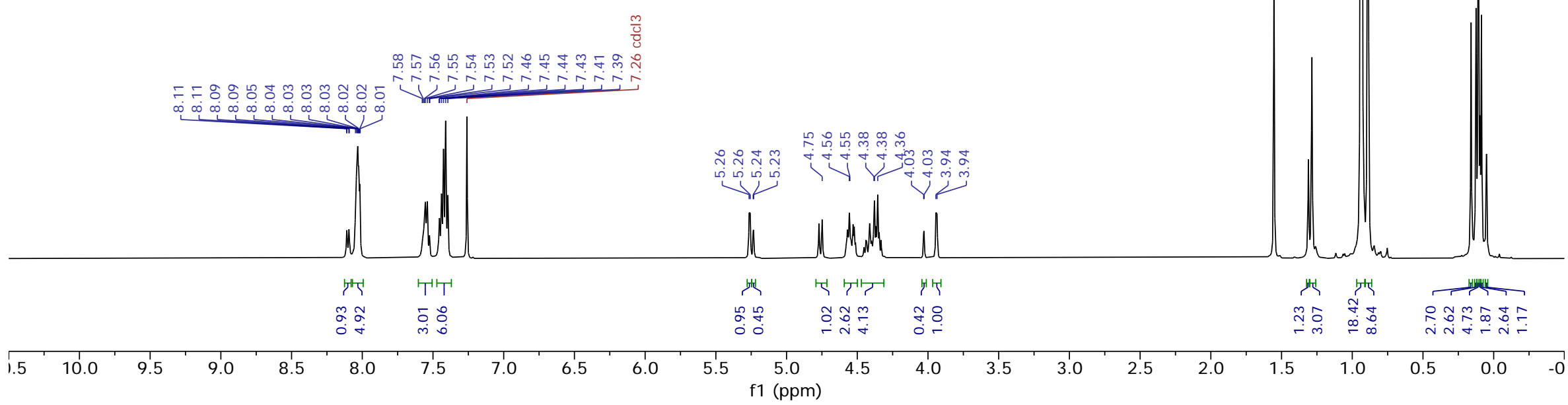


${ }^{13} \mathrm{C}\left\{{ }^{1} \mathrm{H}\right\}-\mathrm{NMR}\left(126 \mathrm{MHz}, \mathrm{CDCl}_{3}\right)$
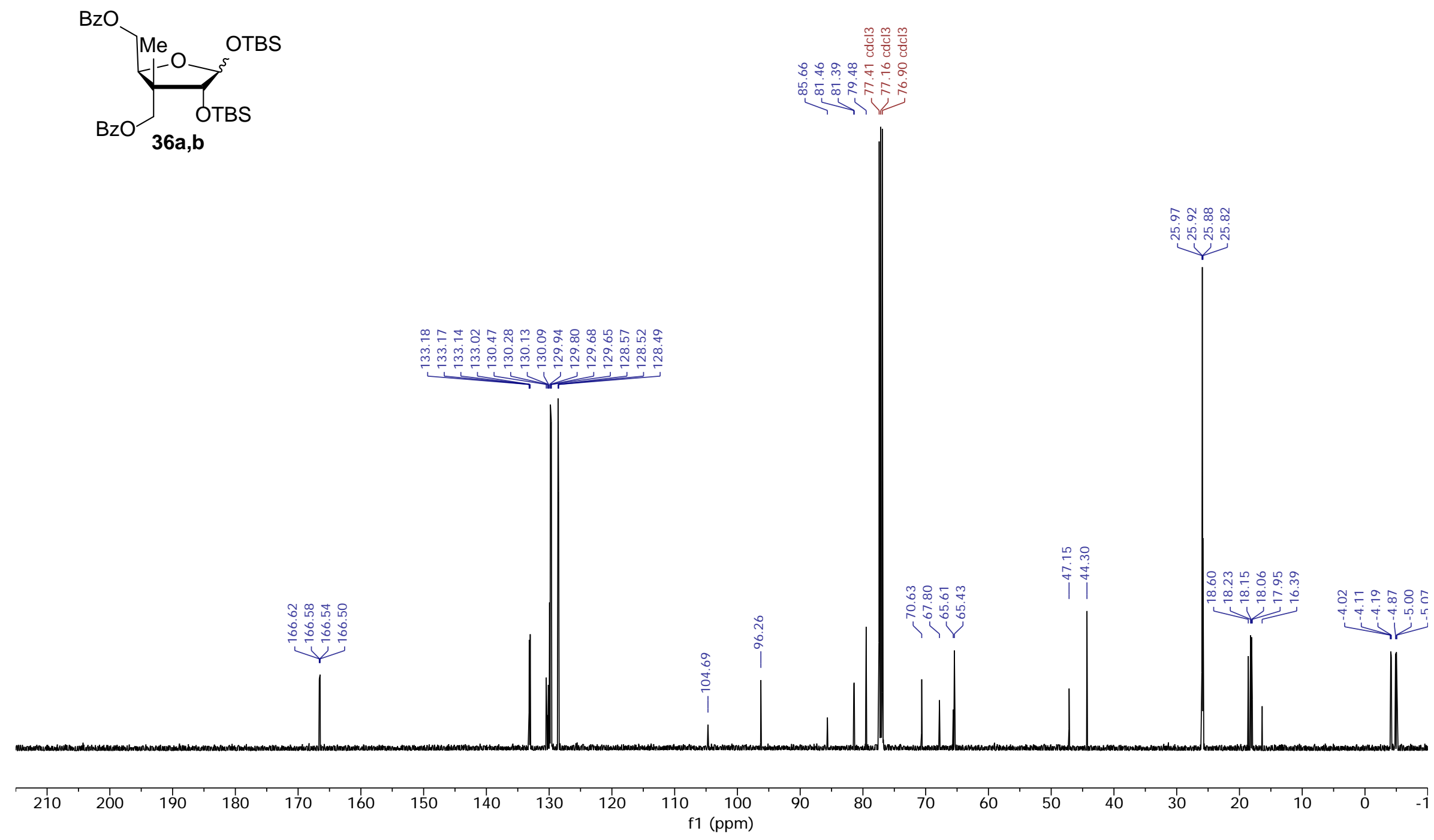
${ }^{1} \mathrm{H}-\mathrm{NMR}\left(500 \mathrm{MHz}, \mathrm{CDCl}_{3}\right.$ )
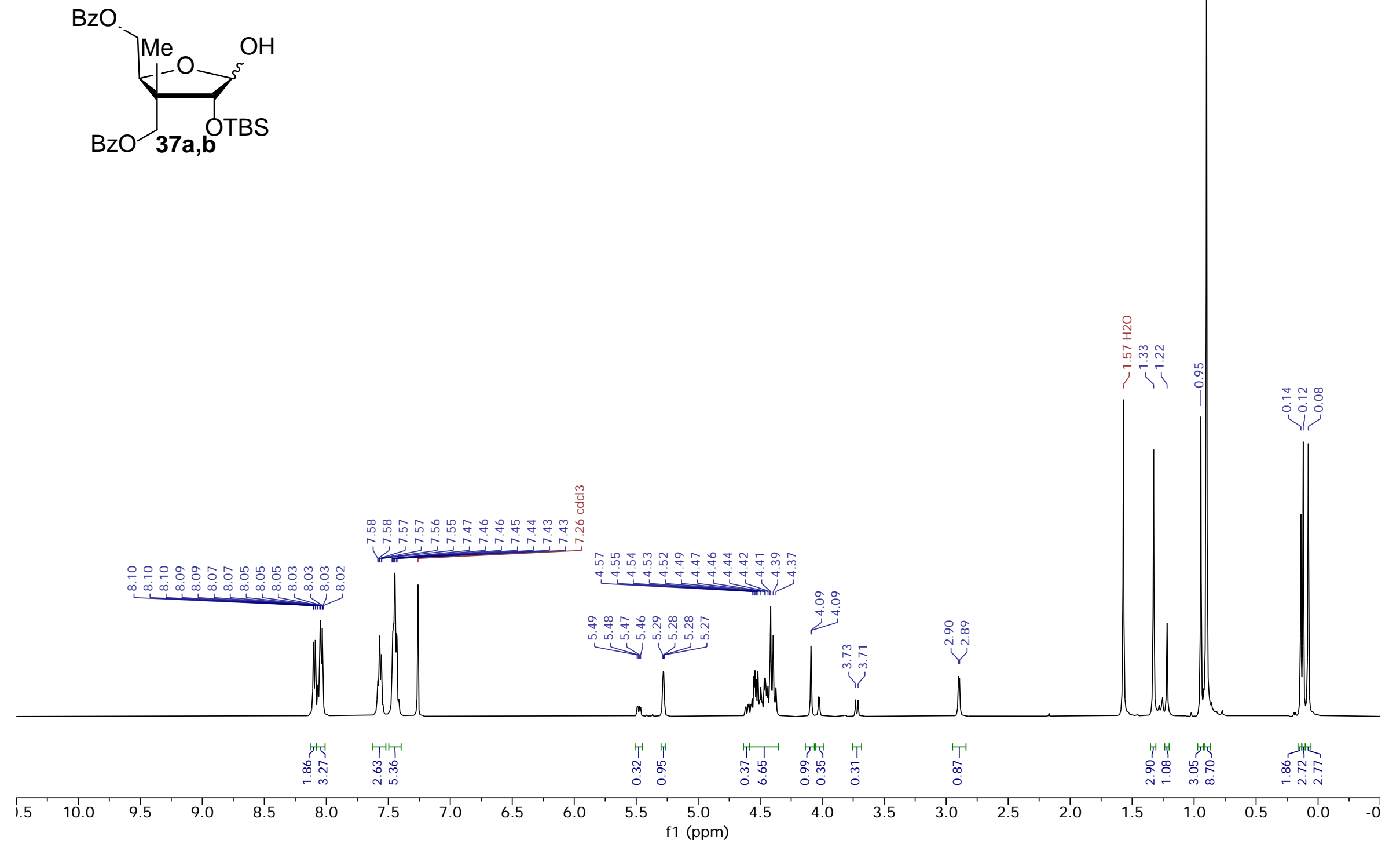
${ }^{13} \mathrm{C}\left\{{ }^{1} \mathrm{H}\right\}-\mathrm{NMR}\left(126 \mathrm{MHz}, \mathrm{CDCl}_{3}\right)$

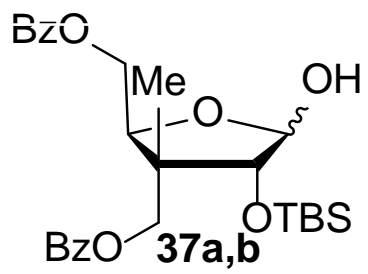

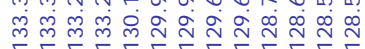

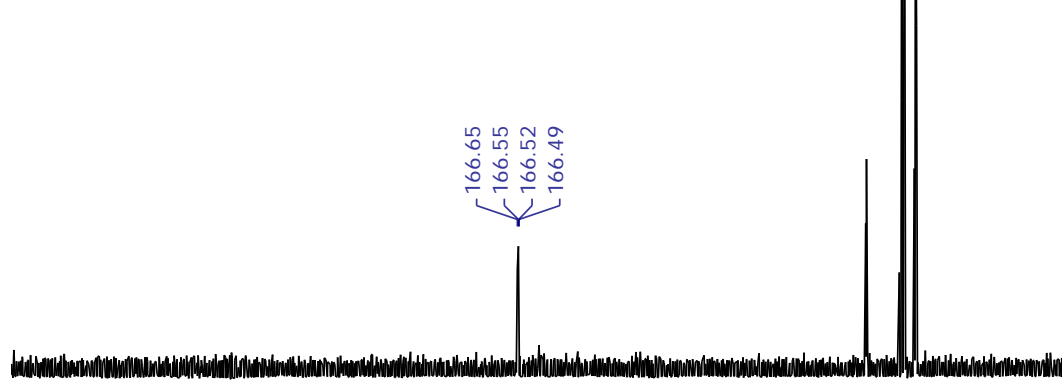


${ }^{1} \mathrm{H}-\mathrm{NMR}$ (500 MHz, $\mathrm{CDCl}_{3}$ )
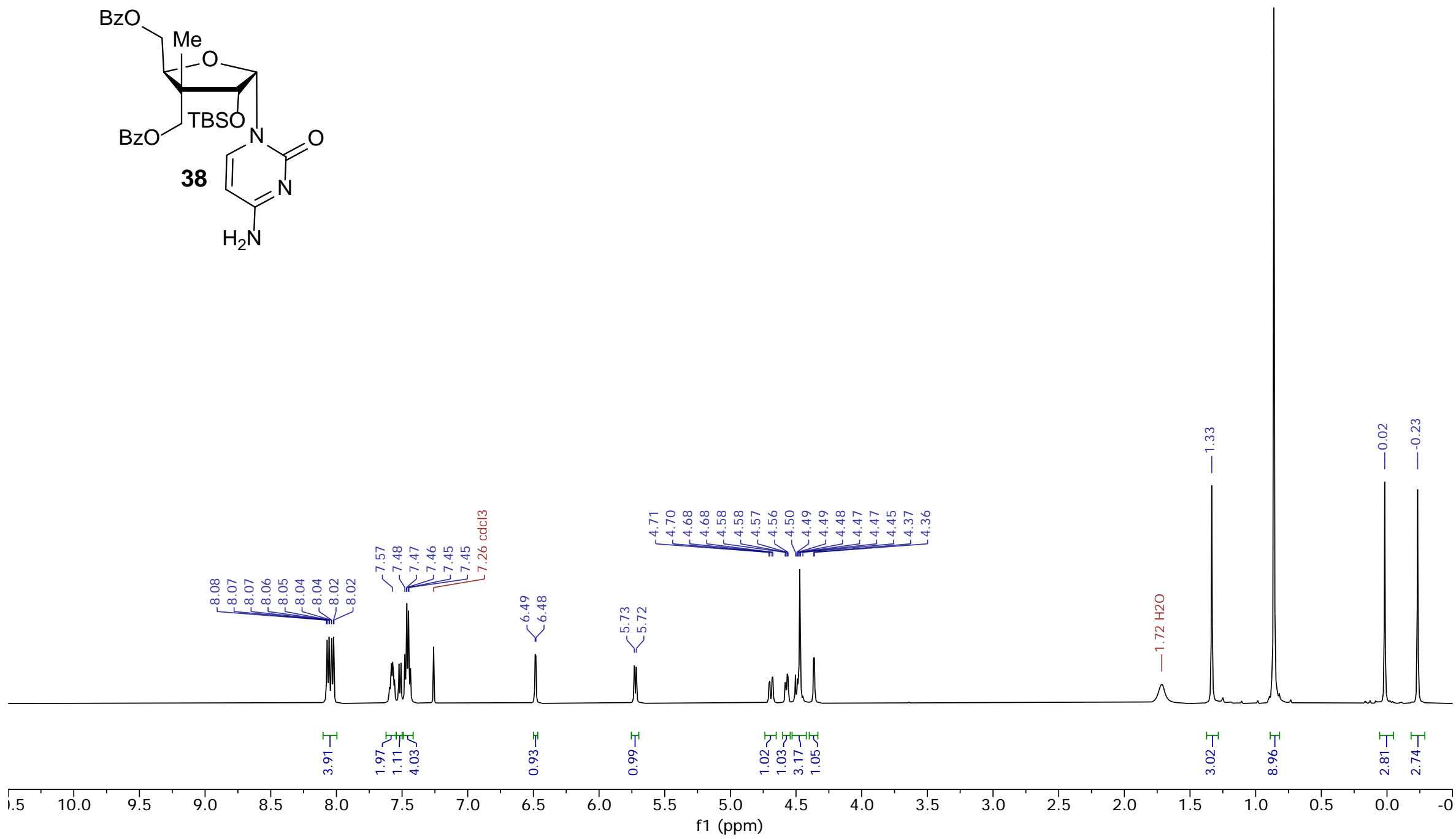
${ }^{13} \mathrm{C}\left\{{ }^{1} \mathrm{H}\right\}-\mathrm{NMR}\left(126 \mathrm{MHz}, \mathrm{CDCl}_{3}\right)$
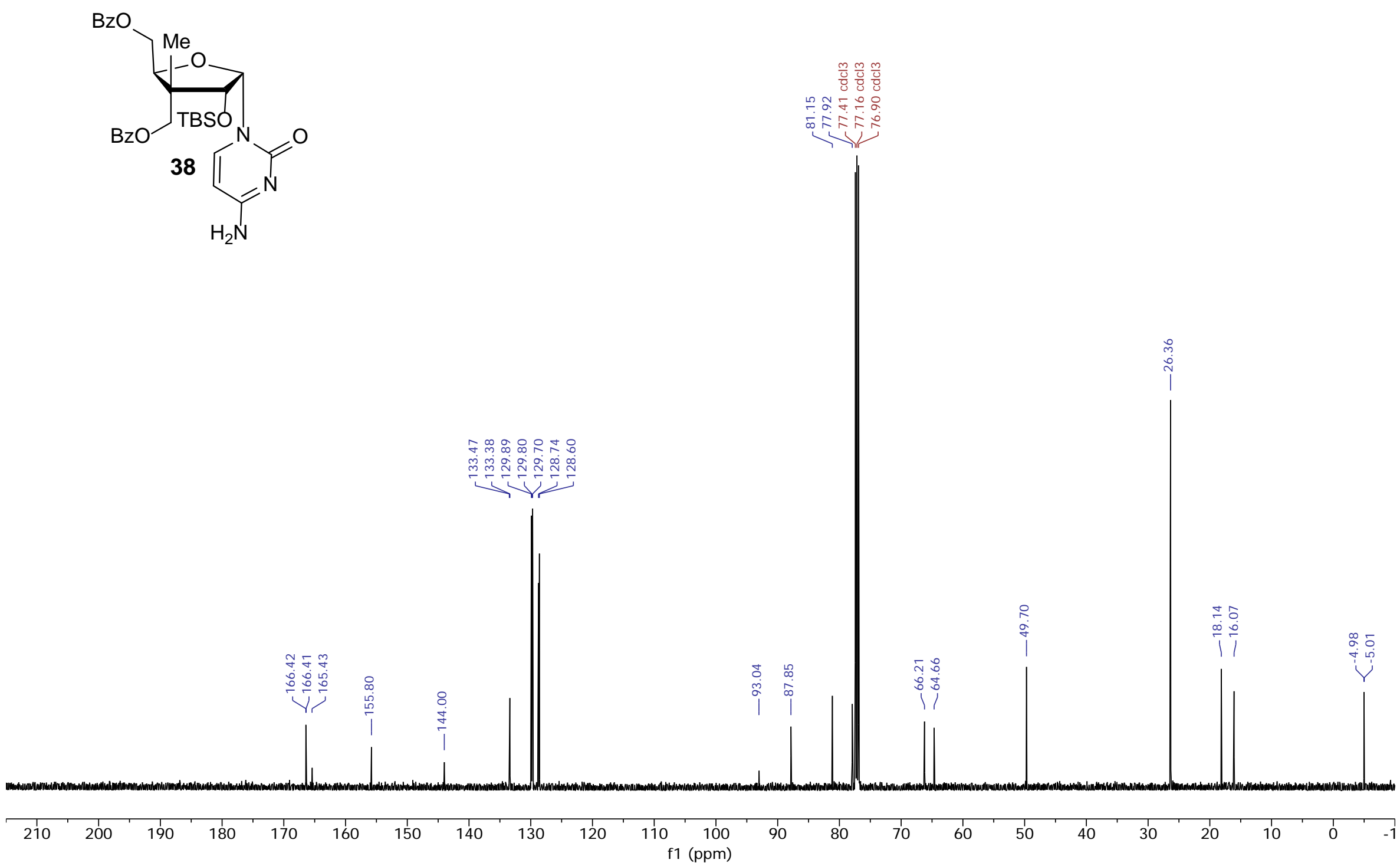
${ }^{1} \mathrm{H}-\mathrm{NMR}\left(500 \mathrm{MHz}, \mathrm{CD}_{3} \mathrm{OD}\right.$ )
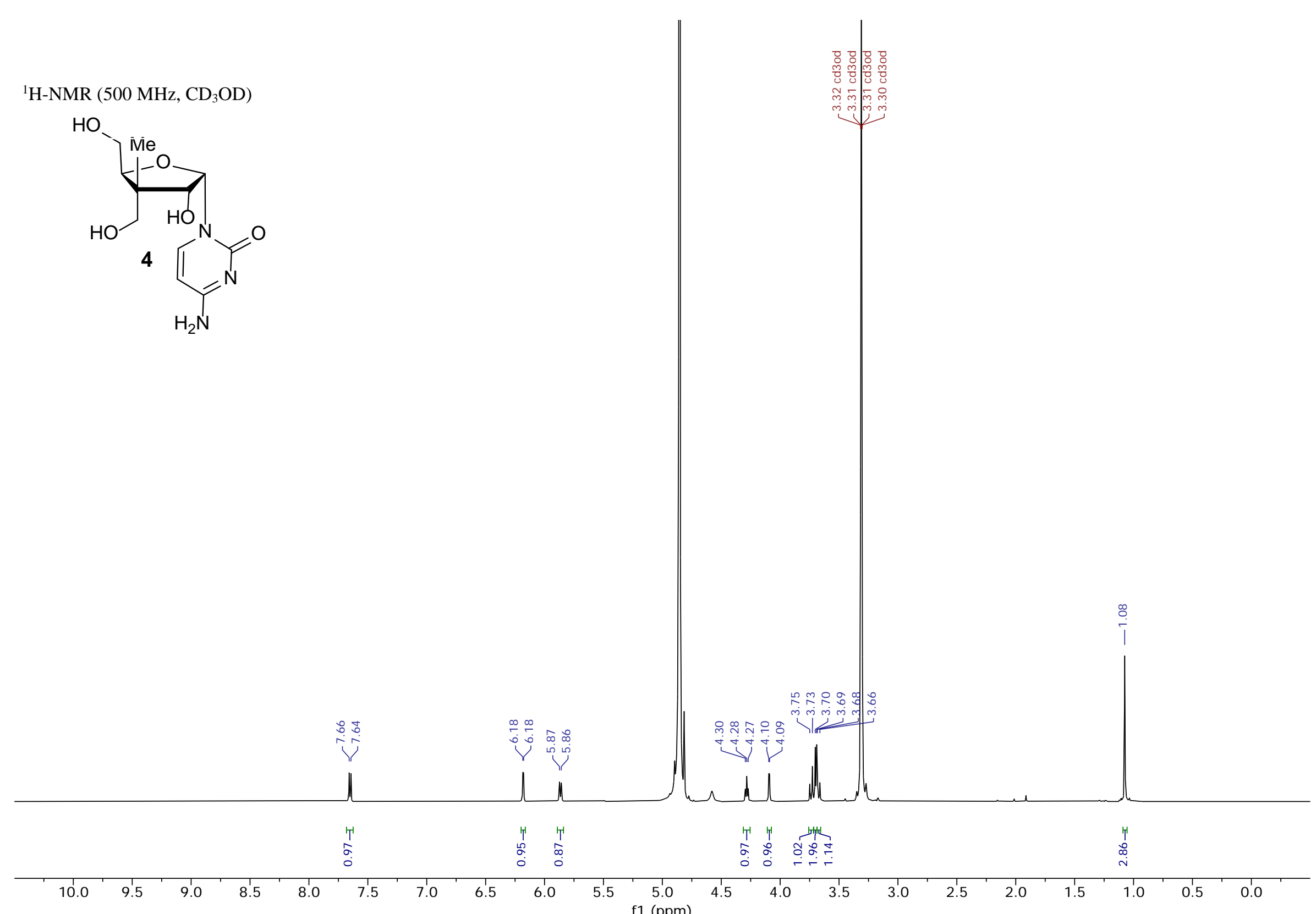
${ }^{13} \mathrm{C}\left\{{ }^{1} \mathrm{H}\right\}$-NMR (126 MHz, CD $\left.{ }_{3} \mathrm{OD}\right)$
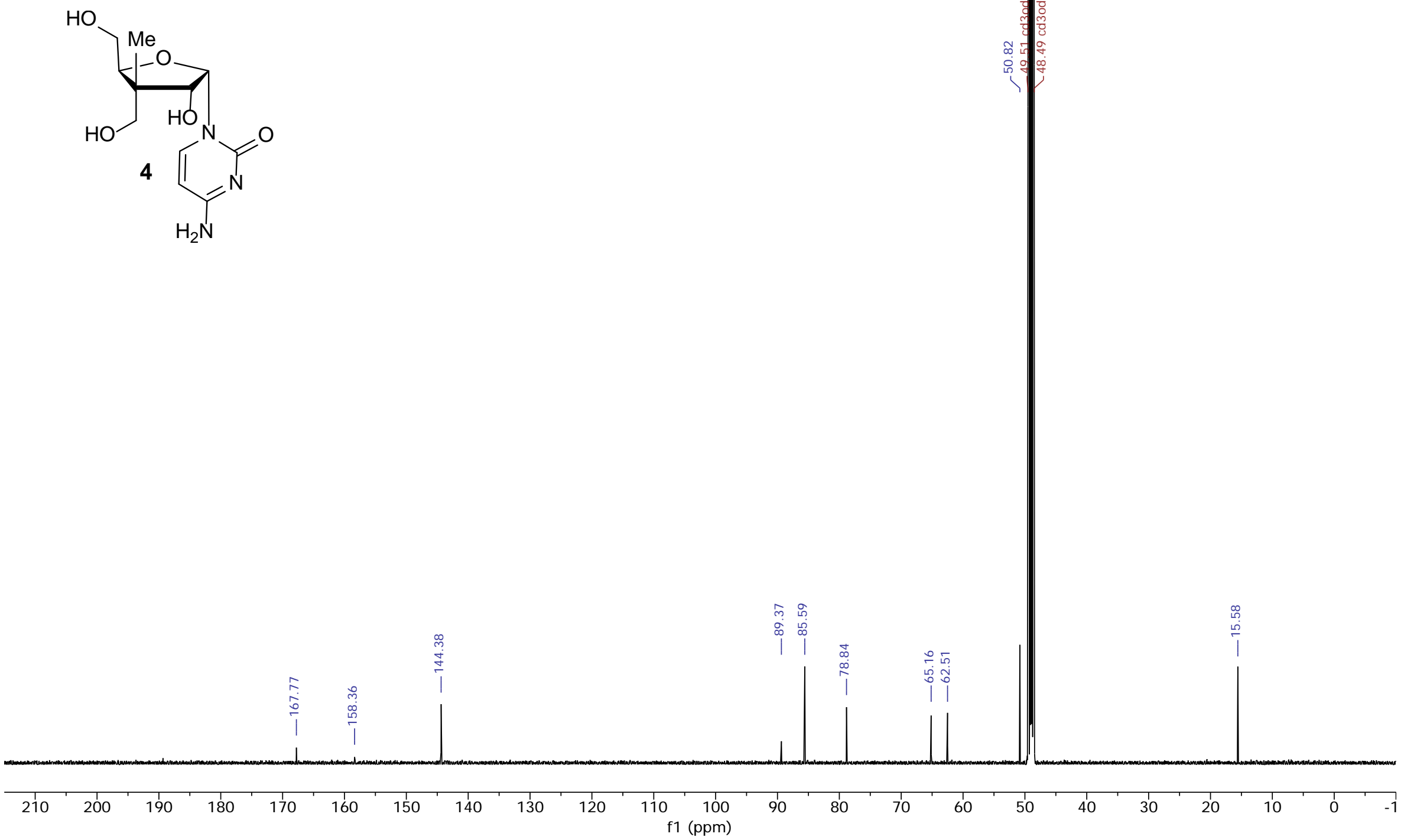
${ }^{1} \mathrm{H}-\mathrm{NMR}\left(500 \mathrm{MHz}, \mathrm{CDCl}_{3}\right)$
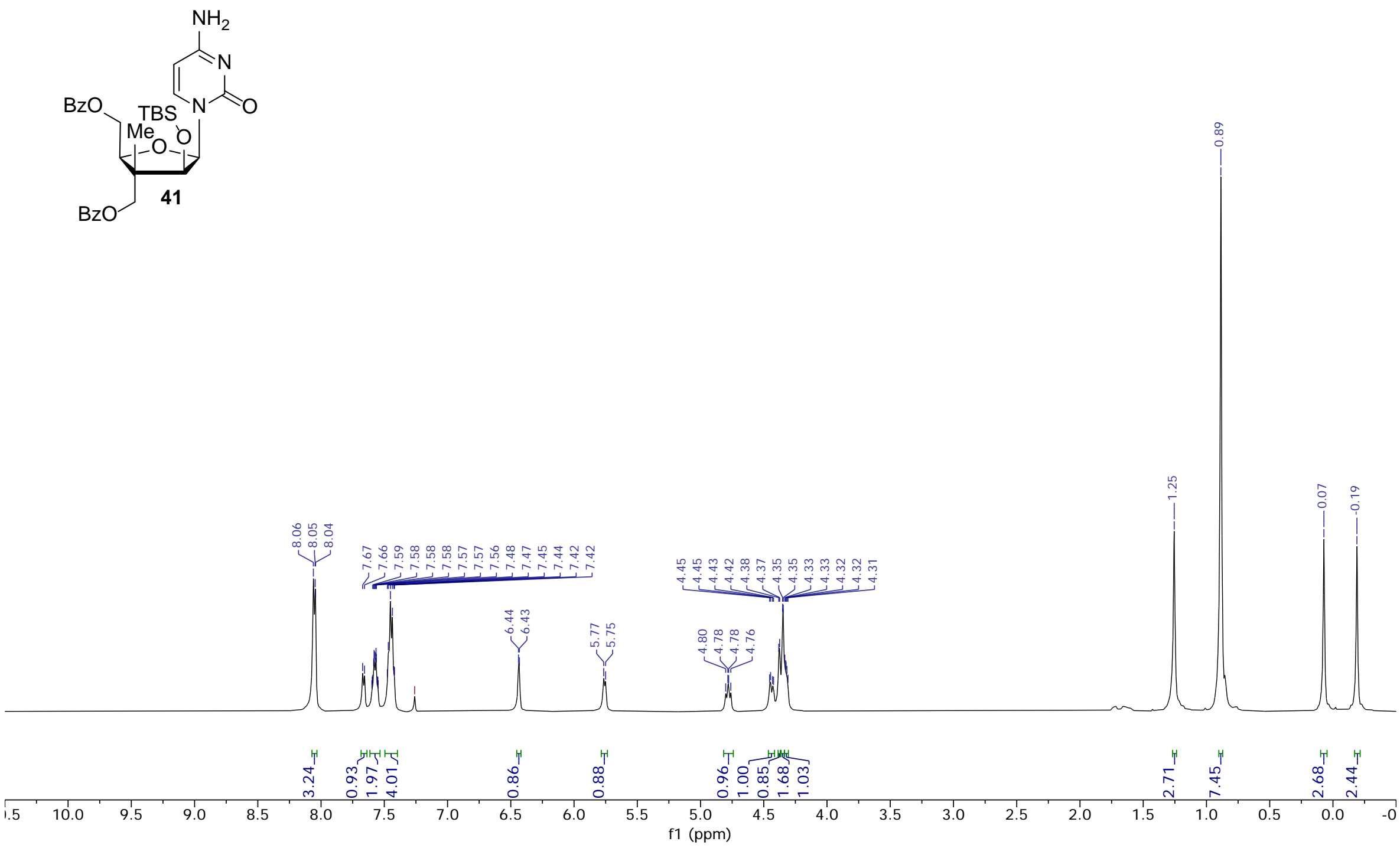
${ }^{13} \mathrm{C}\left\{{ }^{1} \mathrm{H}\right\}-\mathrm{NMR}\left(126 \mathrm{MHz}, \mathrm{CDCl}_{3}\right)$
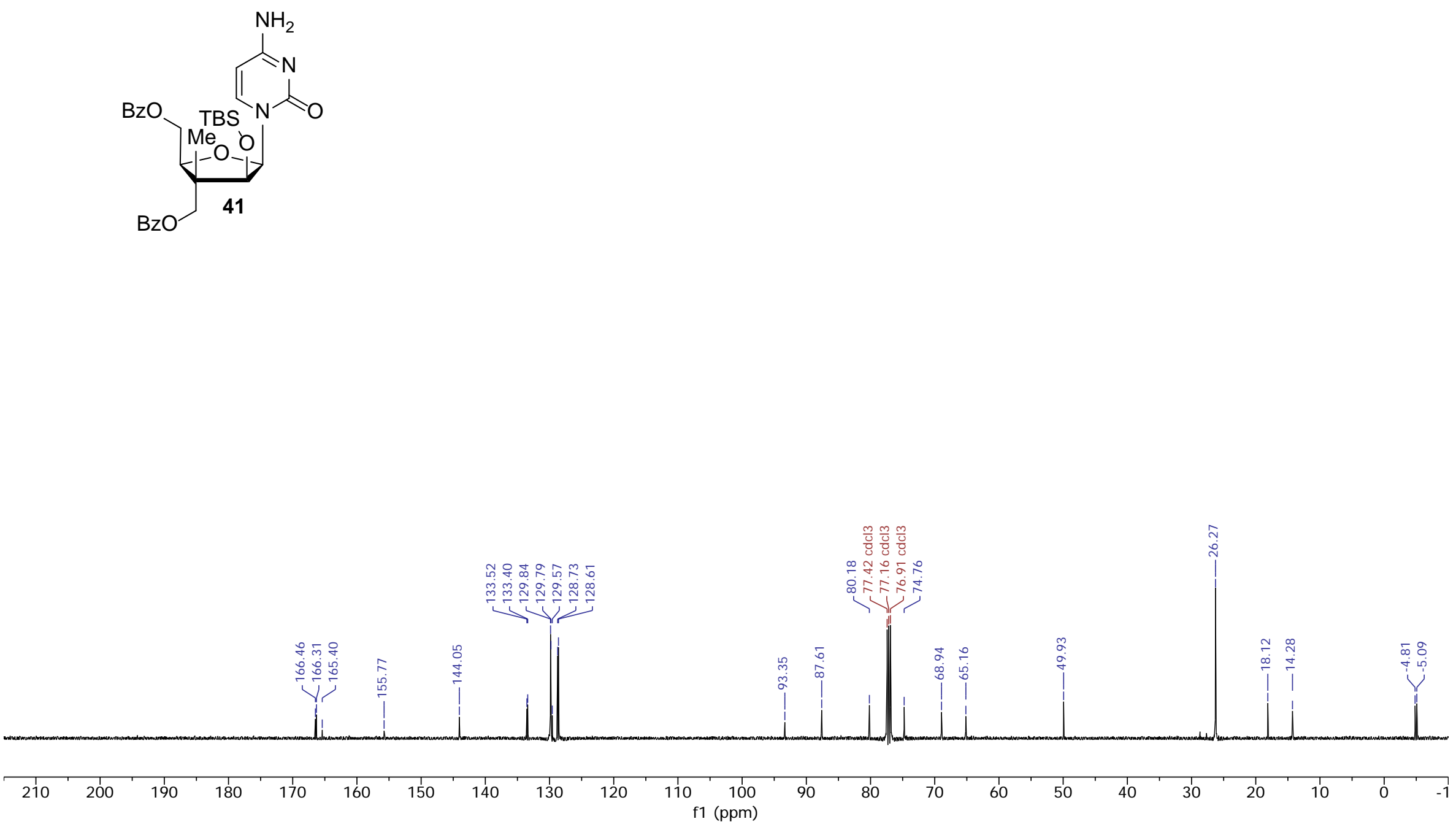
${ }^{1} \mathrm{H}-\mathrm{NMR}\left(500 \mathrm{MHz}, \mathrm{CDCl}_{3}\right.$ )
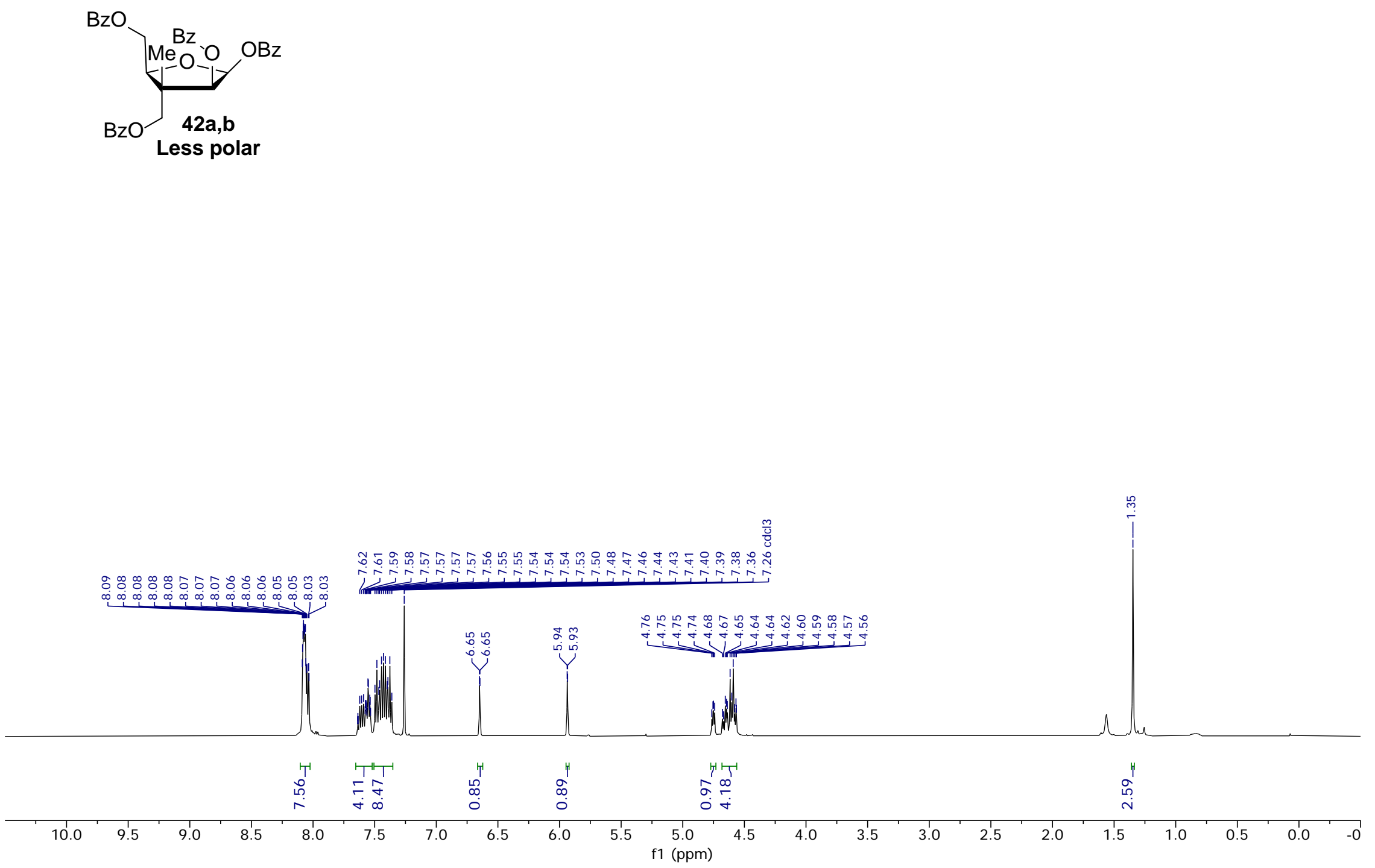
${ }^{13} \mathrm{C}\left\{{ }^{1} \mathrm{H}\right\}$-NMR (126 MHz, $\left.\mathrm{CDCl}_{3}\right)$
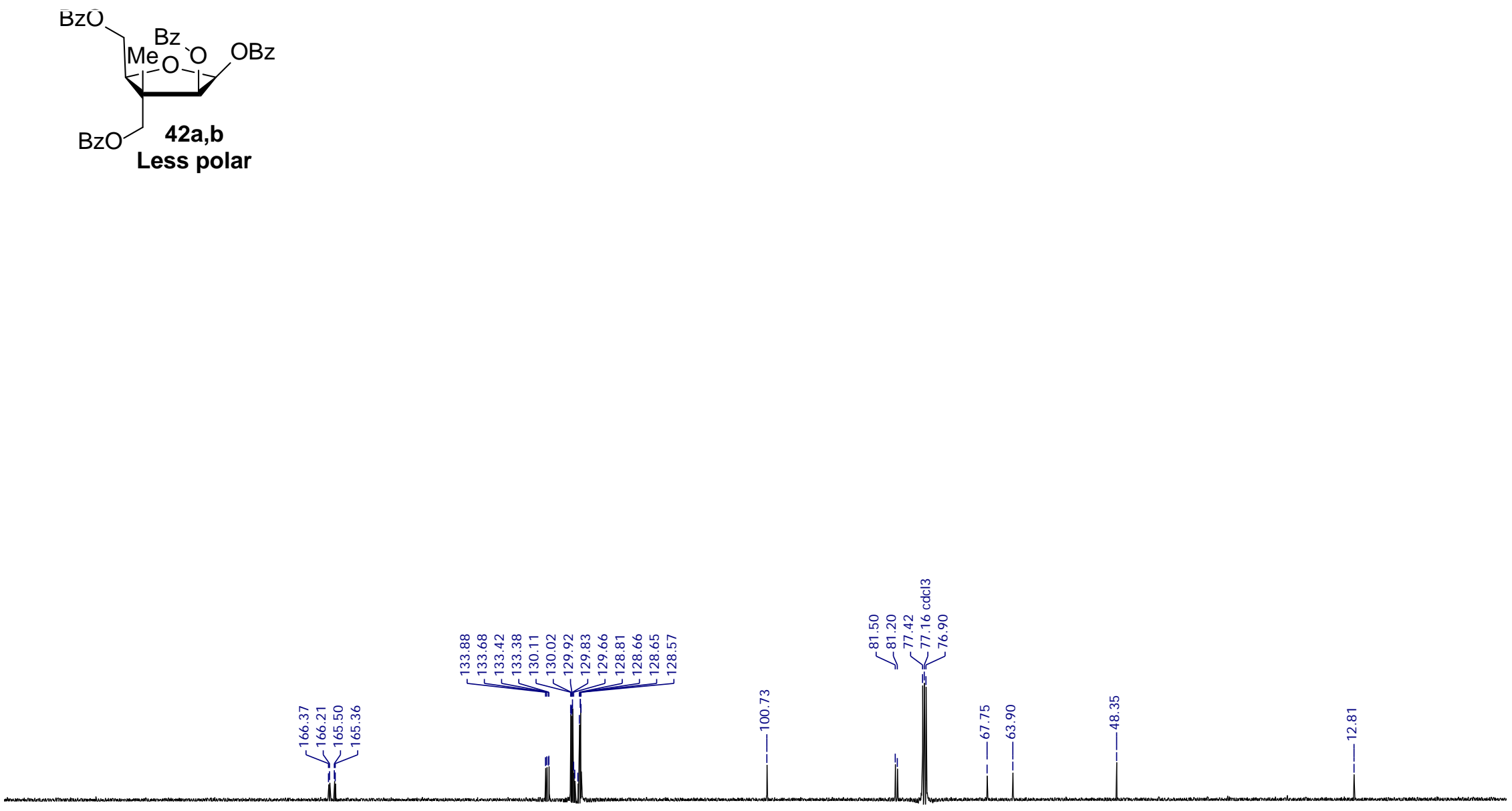
${ }^{1} \mathrm{H}-\mathrm{NMR}\left(500 \mathrm{MHz}, \mathrm{CDCl}_{3}\right)$
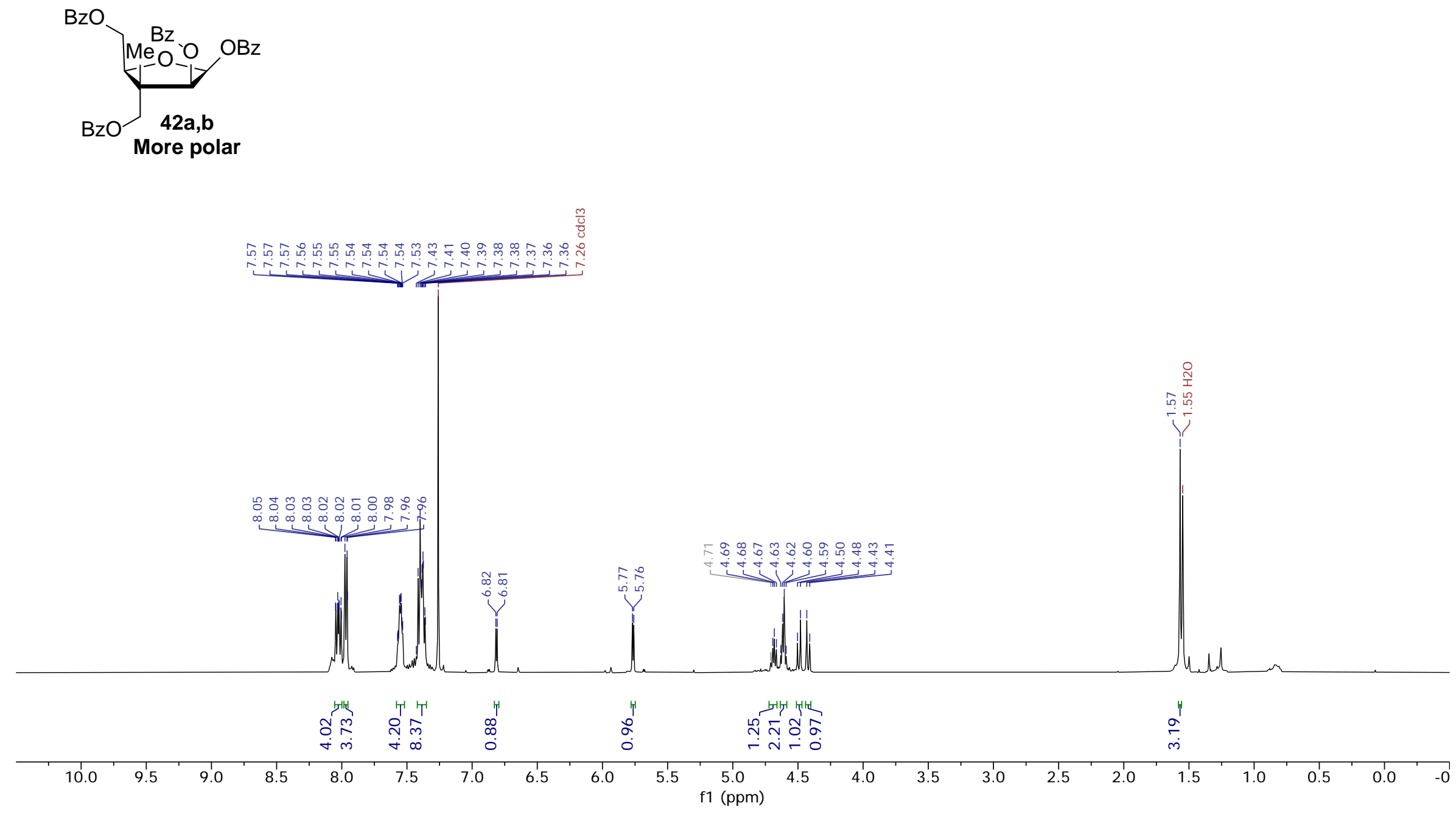
${ }^{13} \mathrm{C}\left\{{ }^{1} \mathrm{H}\right\}$-NMR $\left(126 \mathrm{MHz}, \mathrm{CDCl}_{3}\right)$
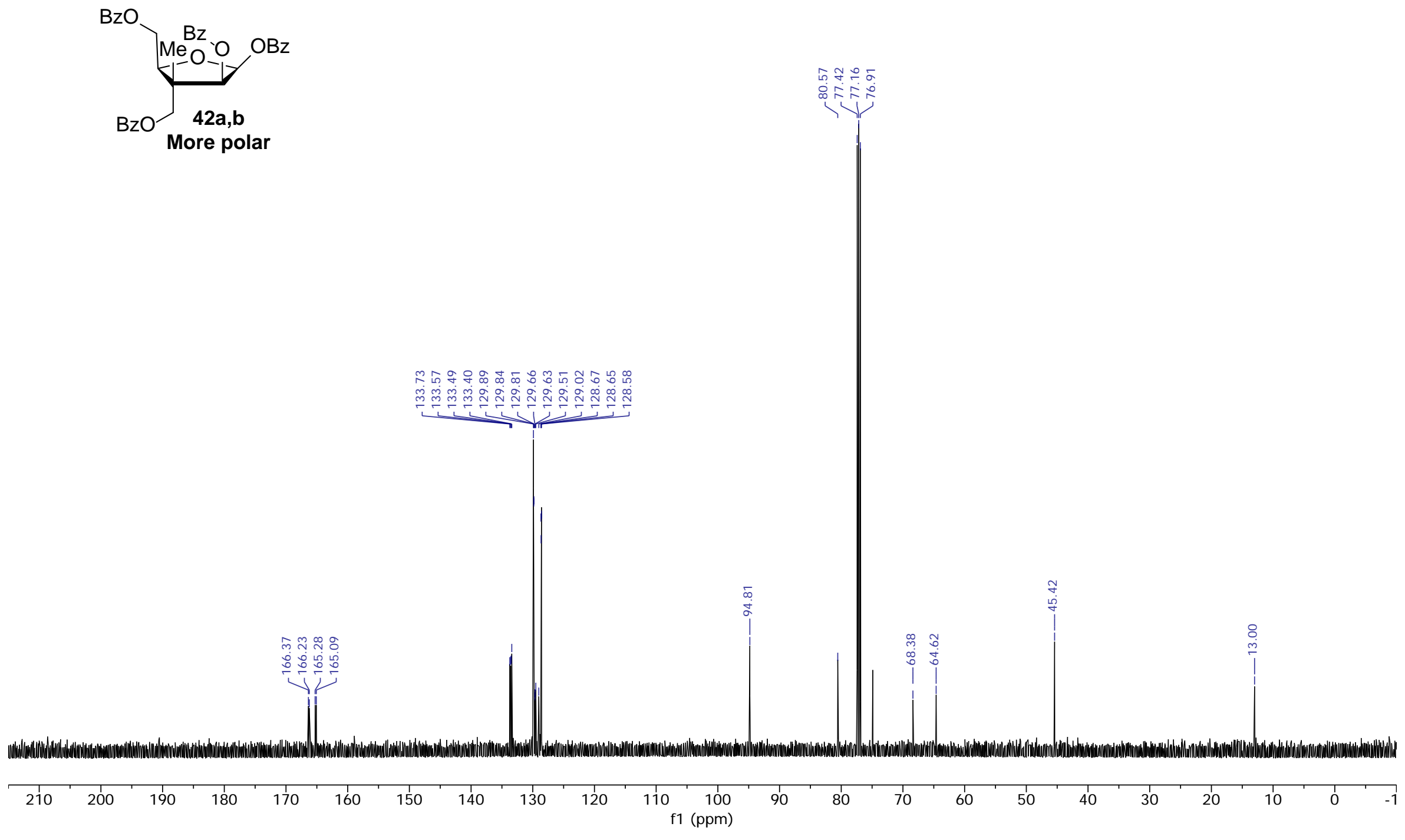
${ }^{1} \mathrm{H}-\mathrm{NMR}\left(500 \mathrm{MHz}, \mathrm{CDCl}_{3}\right)$
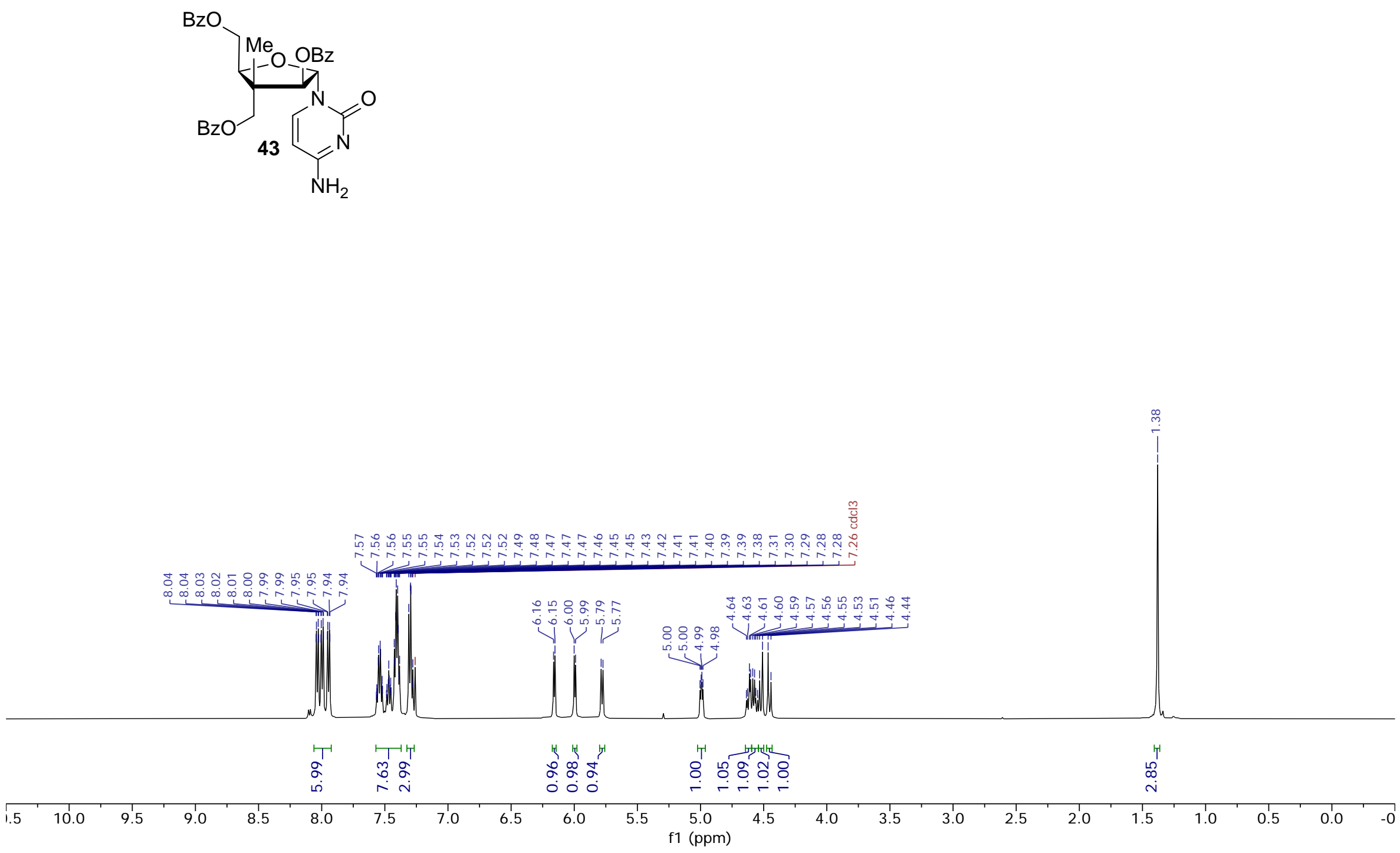
${ }^{13} \mathrm{C}\left\{{ }^{1} \mathrm{H}\right\}-\mathrm{NMR}\left(126 \mathrm{MHz}, \mathrm{CDCl}_{3}\right)$
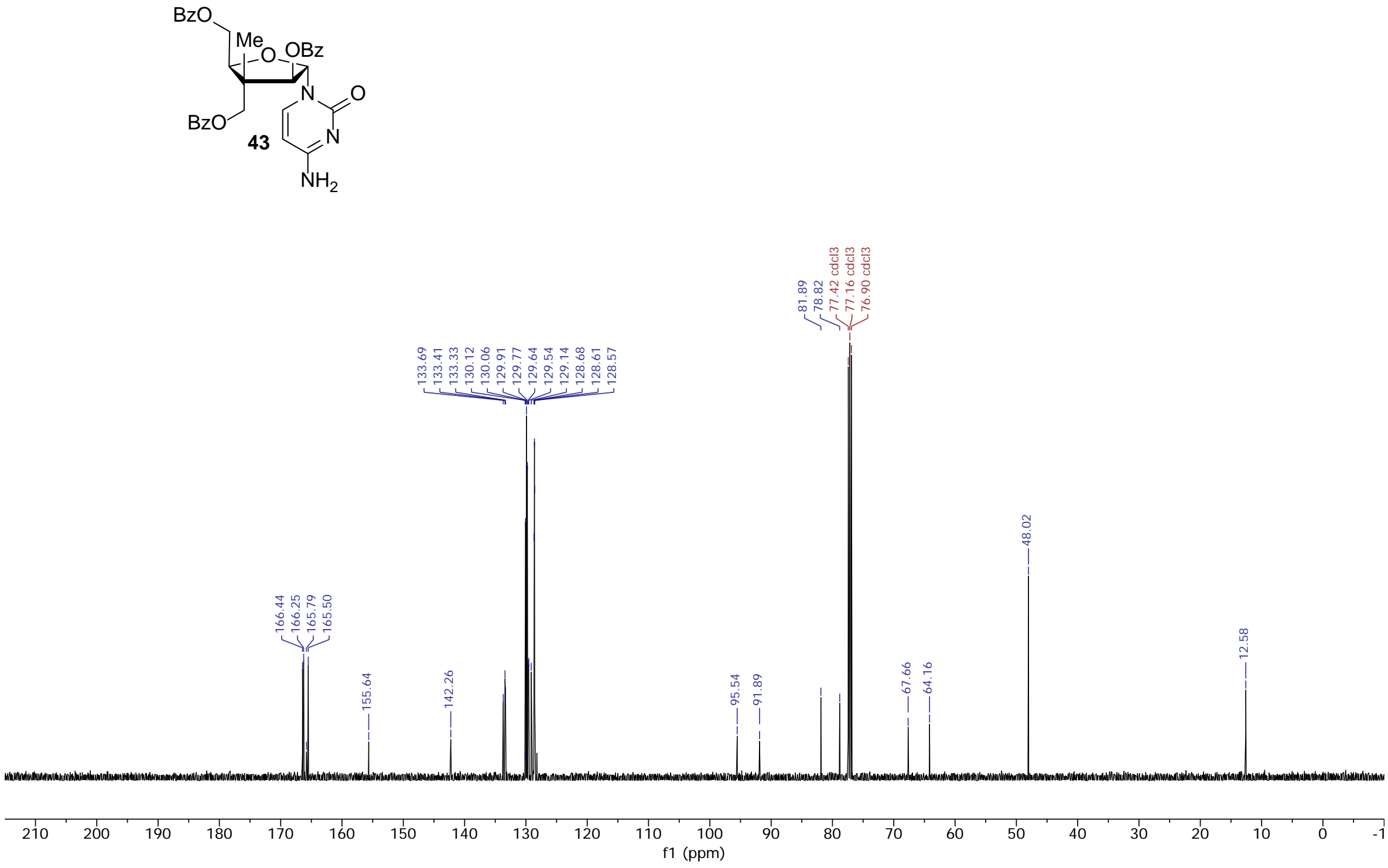
${ }^{1} \mathrm{H}-\mathrm{NMR}\left(500 \mathrm{MHz}, \mathrm{CD}_{3} \mathrm{OD}\right)$
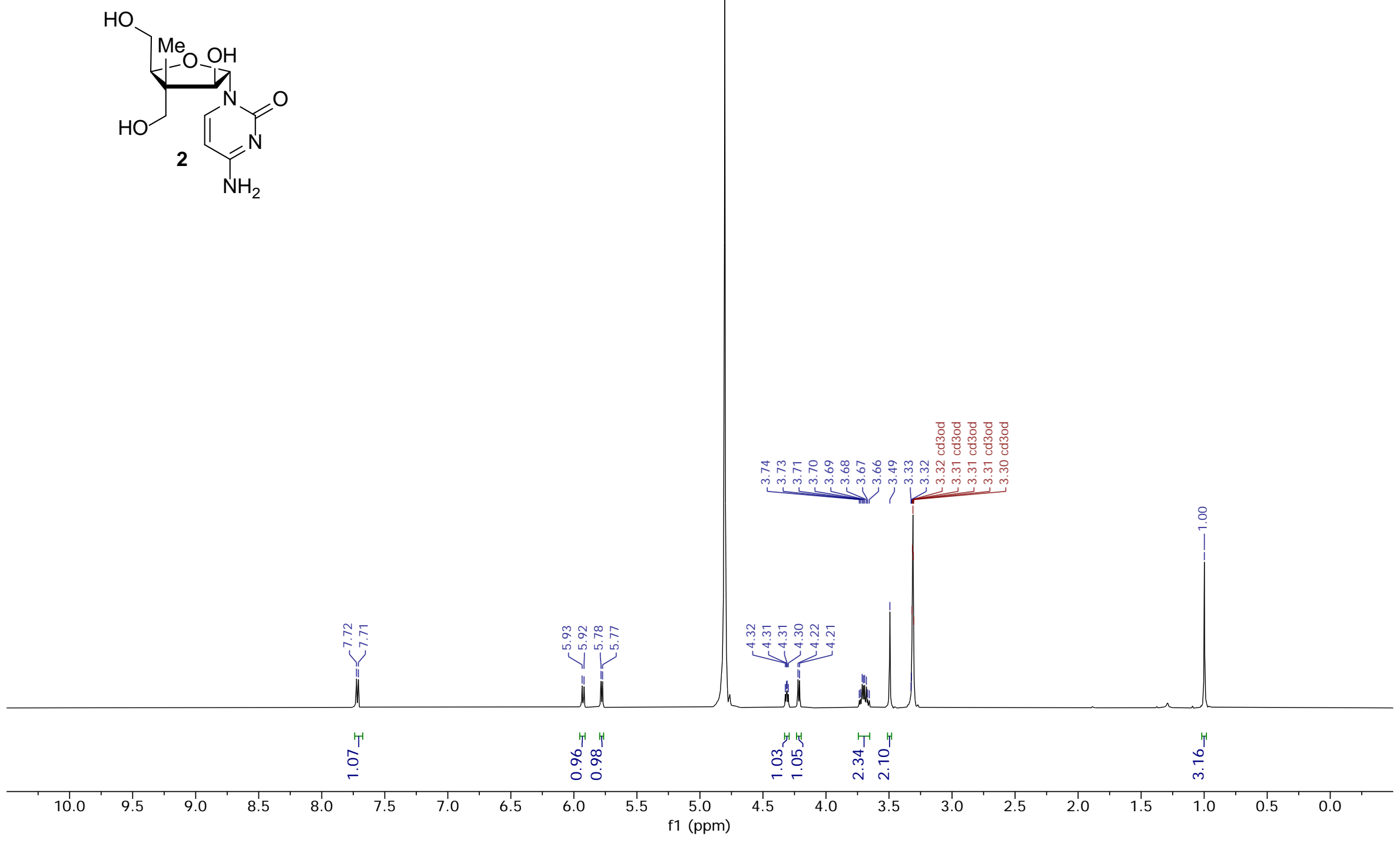
${ }^{13} \mathrm{C}\left\{{ }^{1} \mathrm{H}\right\}-\mathrm{NMR}\left(126 \mathrm{MHz}, \mathrm{CD}_{3} \mathrm{OD}\right)$
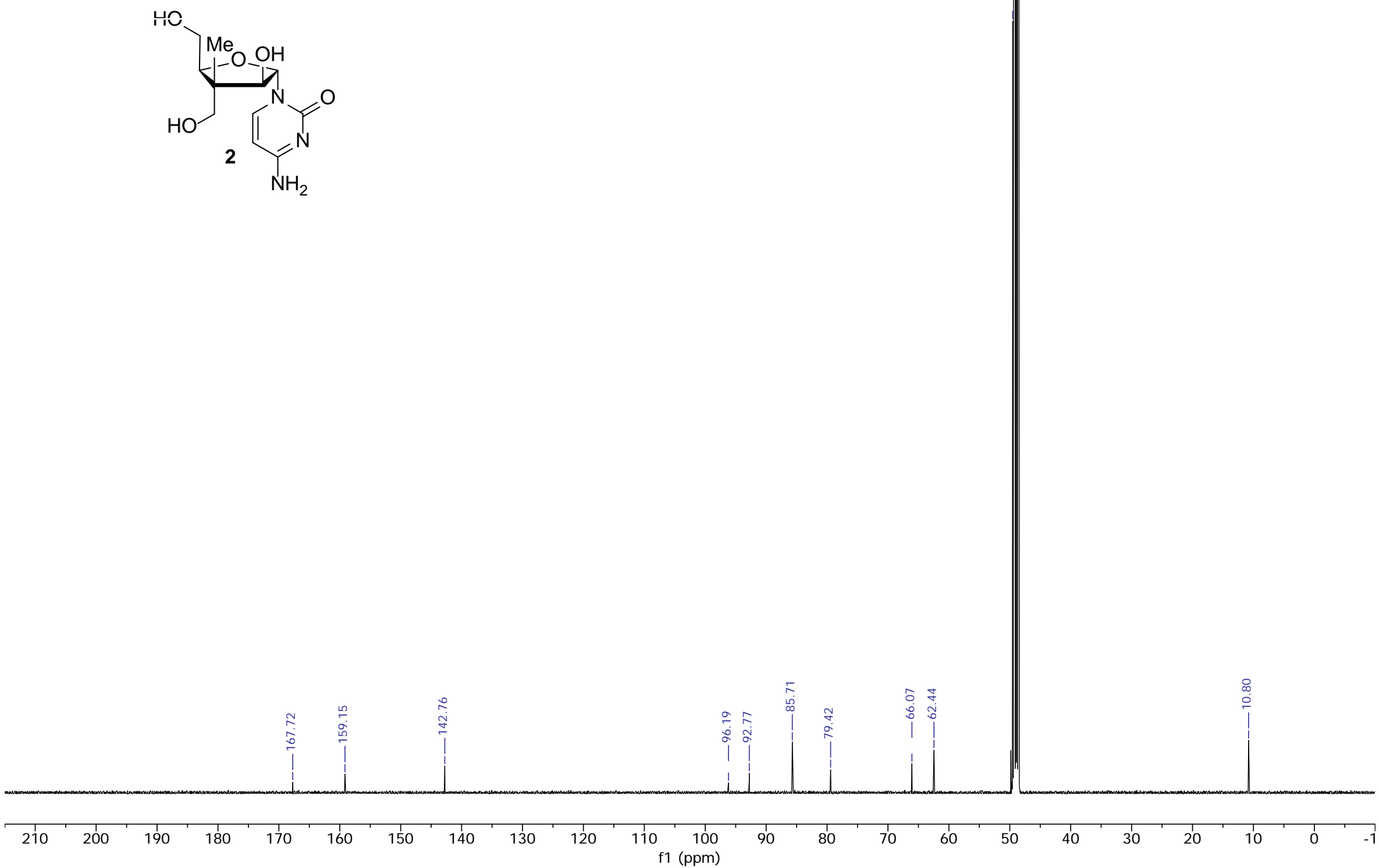
${ }^{1} \mathrm{H}-\mathrm{NMR}\left(500 \mathrm{MHz}, \mathrm{CDCl}_{3}\right.$ )
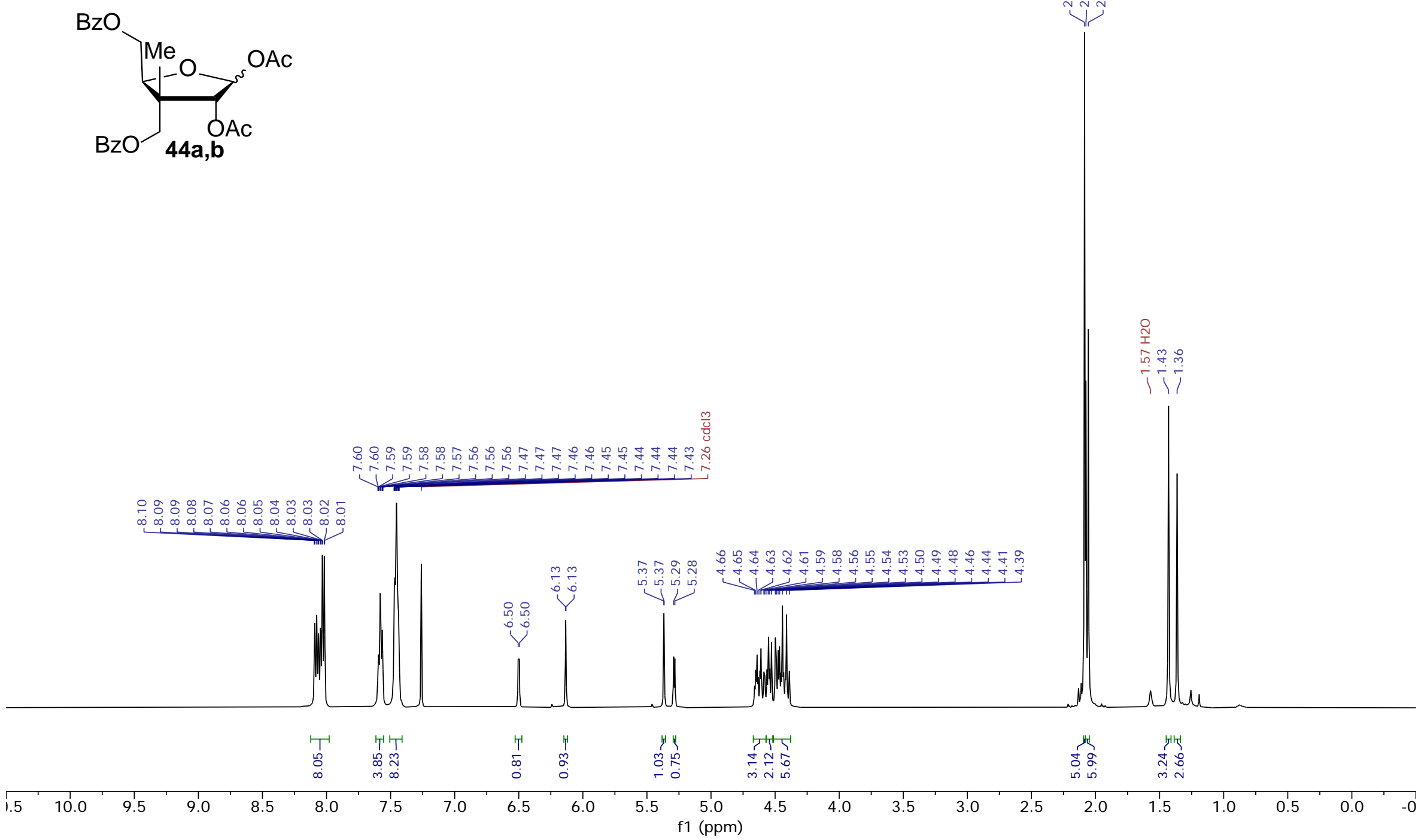
${ }^{13} \mathrm{C}\left\{{ }^{1} \mathrm{H}\right\}-\mathrm{NMR}\left(126 \mathrm{MHz}, \mathrm{CDCl}_{3}\right)$
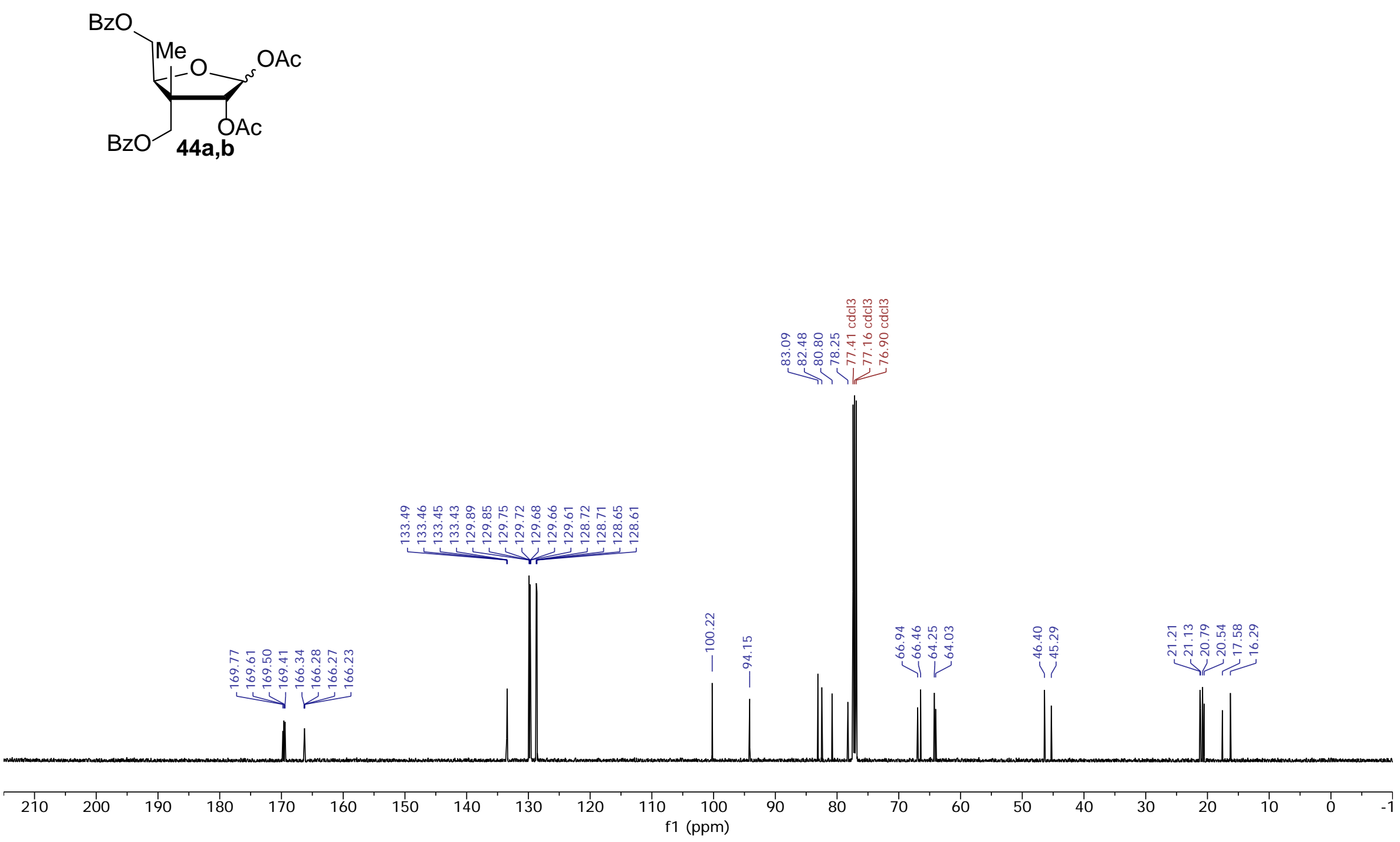
${ }^{1} \mathrm{H}-\mathrm{NMR}\left(500 \mathrm{MHz}, \mathrm{CD}_{3} \mathrm{OD}\right)$
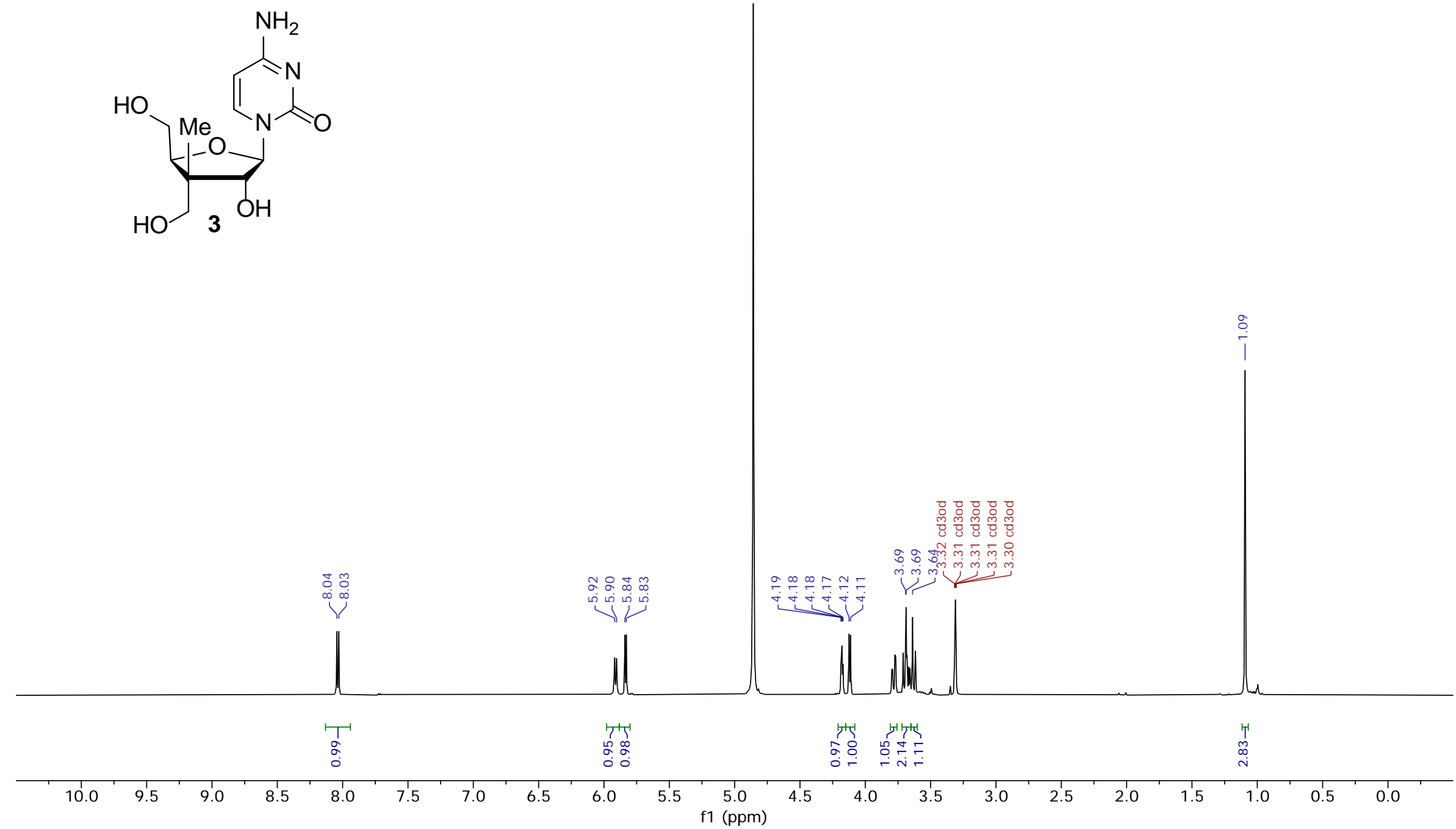
${ }^{13} \mathrm{C}\left\{{ }^{1} \mathrm{H}\right\}-\mathrm{NMR}\left(126 \mathrm{MHz}, \mathrm{CD}_{3} \mathrm{OD}\right)$

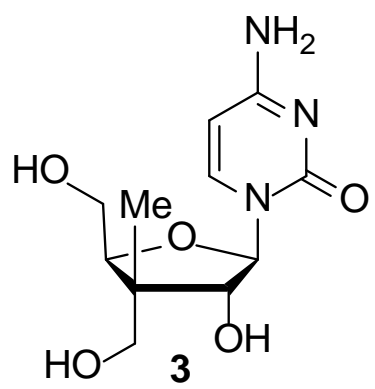

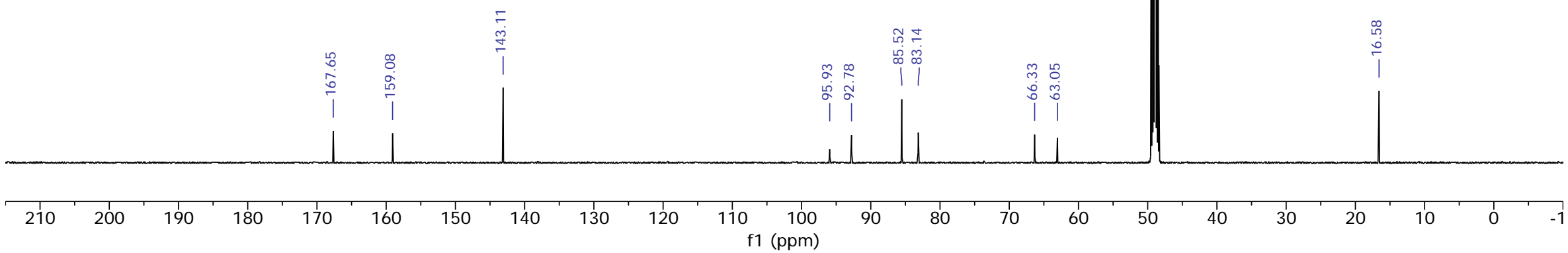




\section{References of SI section}

(1) Gaussian 09, Revision A.02, M. J. Frisch, G. W. Trucks, H. B. Schle-gel, G. E. Scuseria, M. A. Robb, J. R. Cheeseman, G. Scalmani, V.Barone, B. Mennucci, G. A. Petersson, H. Nakatsuji, M. Caricato, X.Li, H. P. Hratchian, A. F. Izmaylov, J. Bloino, G. Zheng, J. L. Son-nenberg, M. Hada, M. Ehara, K. Toyota, R. Fukuda, J. Hasegawa,M. Ishida, T. Nakajima, Y. Honda, O. Kitao, H. Nakai, T. Vreven,J. A. Montgomery, Jr., J. E. Peralta, F. Ogliaro, M. Bearpark, J. J.Heyd, E. Brothers, K. N. Kudin, V. N. Staroverov, R. Kobayashi, J.Normand, K. Raghavachari, A. Rendell, J. C. Burant, S. S. Iyengar,J. Tomasi, M. Cossi, N. Rega, J. M. Millam, M. Klene, J. E. Knox,J. B. Cross, V. Bakken, C. Adamo, J. Jaramillo, R. Gomperts, R. E.Stratmann, O. Yazyev, A. J. Austin, R. Cammi, C. Pomelli, J. W.Ochterski, R. L. Martin, K. Morokuma, V. G. Zakrzewski, G. A.Voth, P. Salvador, J. J. Dannenberg, S. Dapprich, A. D. Daniels, Ö.Farkas, J. B. Foresman, J. V. Orti

(2) BHandHLYP functional is a hybrid functional defined as a half-and-half combination of $\mathrm{HF}$ and

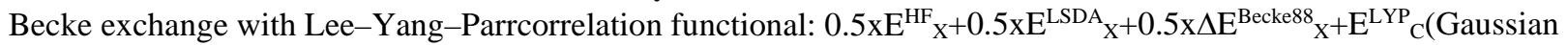
09)

(3) M. Mohr, H. Zipse, D. Marx, M. Parrinello,J. Phys. Chem. A1997,101, 8942-8948.

(4) A. Schäfer, C. Huber, R. Ahlrichs,J. Chem. Phys.1994,100, 5829-5835.

(5) Cossi, M.; Scalmani, G.; Rega, N.; Barone, V. J. Chem. Phys. New developments in the polarizable continuum model for quantum mechanical and classical calculations on molecules in solution; 2002, $117,43$.

(6) Zhao, Y.; Truhlar, D. G. Phys. Chem. Chem. Phys. Computational characterization and modeling of buckyball tweezers: density functional study of concave-convex pi...pi interactions; 2008, 10, 2813.

(7) Ribeiro, R. F.; Marenich, A. V.; Cramer, C. J.; Truhlar, D. G. J. Phys. Chem. B Use of solutionphase vibrational frequencies in continuum models for the free energy of solvation; 2011, 115, 14556. 